\title{
Inverse Visual Question Answering with Multi-Level Attentions
}

\author{
by \\ Yaser Alwattar \\ A thesis submitted to the \\ Faculty of Graduate and Postdoctoral Affairs \\ in partial fulfillment of the requirements for the degree of
}

Master of Computer Science with Data Science Specialization

Ottawa-Carleton Institute for Computer Science

School of Computer Science

Carleton University

Ottawa, Ontario

August, 2019

(C) Copyright

Yaser Alwattar, 2019 


\section{Abstract}

Inverse Visual Question Answering (iVQA) is a contemporary task emerged from the need for improving visual and language understanding. It tackles the challenging problem of generating a corresponding question for a given image-answer pair. Current state-of-the-art iVQA models use the conventional way of representing images by using a convolutional neural network $(\mathrm{CNN})$ to extract visual features. Although some models leverage semantic concepts as an enhancement for the answer cue, they give the same importance weights to these concepts without considering their correlation with the answers. Moreover, the existing iVQA models mainly rely on the conventional recurrent neural networks for question modelling, while the attentionbased sequence learning mechanism for question modelling, which could help to reduce model parameters, remains unexplored. In this thesis, we address these issues by developing two novel deep multi-level attention models for the task of inverse visual question answering. The first static multi-level attention model (SMLA) generates regional visual and semantic features at the level of objects and then enhances them with the answer cue by using attention mechanisms. Three levels of attentions are employed in this model to capture various aspects of the image and answer relationship. The second proposed approach is a dynamic multi-level attention model (DMLA) that dynamically changes its attention weights based on the current generation state. This model adopts attention modules for both question encoding and decoding. We eval- 
uate the proposed models on the VQA V1 dataset. We examined their performance by using a number of popular evaluation metrics, namely BLEU-(1,2,3,4), METEOR, ROUGE-L, and CIDEr. The effectiveness of our approaches is proved by achieving the state-of-the-art performance in comparison to other competitors. 


\section{Acknowledgements}

I would like to thank my supervisor, Professor Yuhong Guo, for the valuable ideas and feedback to complete this thesis. I really appreciate her support and help during my Master's studies. I would also like to thank my defence committee members and their helpful comments to improve my thesis. 


\section{Contents}

Abstract ii

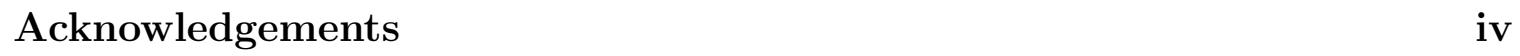

Table of Contents vii

List of Tables viii

List of Figures xii

Nomenclature xiii

$\begin{array}{lll}1 & \text { Introduction } & 1\end{array}$

\begin{tabular}{lll}
\hline 2 & Background & 7
\end{tabular}

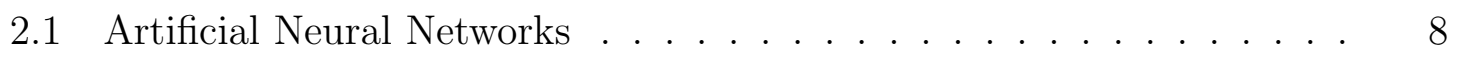

$2.1 .1 \quad$ Feed-forward Neural Networks . . . . . . . . . . . . . . . . . . 8

2.1 .2 Activation Function . . . . . . . . . . . . . . . . . . . . . . . . 9

$2.1 .3 \quad$ Training Neural Networks

2.2 Convolutional Neural Networks . . . . . . . . . . . . . . . . . . . . . 12

2.3 Recurrent Neural Networks. . . . . . . . . . . . . . . . . . . . . . . . . 18

2.3 .1 Gated Recurrent Units (GRUs) . . . . . . . . . . . . . 20 


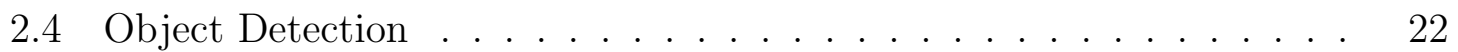

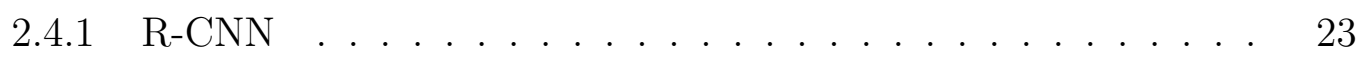

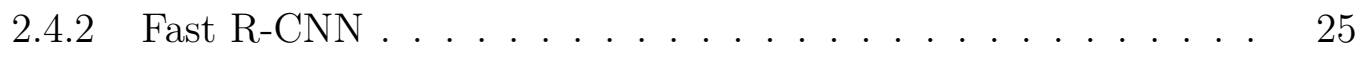

2.4 .3 Faster R-CNN . . . . . . . . . . . . . . . . . . . . . 25

2.5 Multi-modal Feature Fusion . . . . . . . . . . . . . . . . . . . . 31

$2.5 .1 \quad$ Multi-modal Low-rank Bilinear Pooling . . . . . . . . . . . 33

2.5 .2 Multi-modal Factorized Bilinear Pooling . . . . . . . . . . 34

\begin{tabular}{lll}
\hline 3 & Related Work & 36
\end{tabular}

3.1 Image Caption Generation . . . . . . . . . . . . . . . . . . . 36

$3.2 \quad$ Visual Question Answering $\ldots \ldots \ldots \ldots \ldots$

3.3 Visual Question Generation . . . . . . . . . . . . . . . . . . . . 39

3.4 iVQA Models $\ldots \ldots \ldots \ldots$

$3.5 \quad$ Attention for Sequence Learning . . . . . . . . . . . . . . . 43

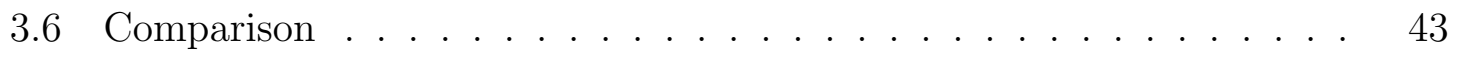

\begin{tabular}{|lll}
4 & The Proposed Models & 46
\end{tabular}

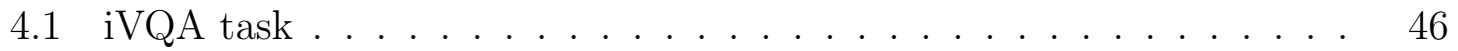

4.2 iVQA Models with Multi-level Attentions . . . . . . . . . . . . 47

4.2 .1 The SMLA Model . . . . . . . . . . . . . . . . . . . . . 48

4.2 .1 .1 Semantic Answer Encoder . . . . . . . . . . . . . 49

$4.2 .1 .2 \quad$ Semantic and Visual Image Encoder . . . . . . . . 50

4.2 .1 .3 Visual and Semantic Attentions . . . . . . . . . . 52

4.2 .1 .4 Multi-Modal Feature Fusion . . . . . . . . . . . . 54

4.2 .1 .5 MFB Attention . . . . . . . . . . . . . 55

$4.2 .1 .6 \quad$ Recurrent Question Generation . . . . . . . . . 57

4.2 .2 The DMLA Model . . . . . . . . . . . . . . . . . . . . . . . . 59 
4.2.2.1 GRU-free Recurrent Question Generation . . . . . . 59

4.3 Training . . . . . . . . . . . . . . . . . . . . . 64

4.4 Inference . . . . . . . . . . . . . . . . . . . . . 65

\begin{tabular}{lll}
\hline 5 & Evaluation Metrics & 66
\end{tabular}

5.1 BLEU . . . . . . . . . . . . . . . . . . 66

5.2 METEOR . . . . . . . . . . . . . . . . . . . . . 69

5.3 ROUGE $\ldots \ldots \ldots \ldots \ldots$. . . . . . . . . . . . . . . . . . . . . . .

5.4 CIDEr . . . . . . . . . . . . . . . . . . . 73

\begin{tabular}{lll}
\hline 6 & Experiments & 77
\end{tabular}

6.1 Experimental Setting . . . . . . . . . . . . . . . . . . . . 77

6.1 .1 Dataset . . . . . . . . . . . . . . . . . 77

6.1 .2 Implementation Details . . . . . . . . . . . . . . . . 78

6.1 .3 Model Evaluation . . . . . . . . . . . . . . . . . . . . . . 79

6.2 Quantitative Analysis . . . . . . . . . . . . . . . . . . . 80

6.3 Ablation Study $\ldots \ldots \ldots$. . . . . . . . . . . . . . . . . . . . . . . . 81

6.4 Qualitative Analysis . . . . . . . . . . . . . . . . . 85

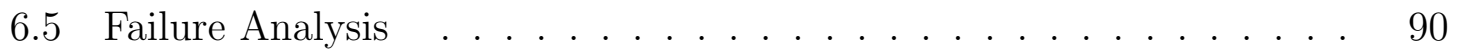

\begin{tabular}{lll}
\hline & Conclusion and Future Work & 93
\end{tabular} 


\section{List of Tables}

6.1 Overall performance comparison between our iVQA models and other baseline and state-of-the-art models on the popular Karpathy test split. Our models achieve best results on most evaluation metrics. The numbers in bold represent the highest scores (best performance). . . . . . 80

6.2 Performance comparison of our SMLA model variants. . . . . . . . . 83

6.3 Performance comparison of our DMLA model variants. . . . . . . . . 85

6.4 Qualitative results of our full SMLA model including a visualization of the weights generated from visual attention $A t t_{v}$ and MFB attention $A t t_{m f b}$ with the top-5 semantic objects selected by our semantic attention module $A t t_{s}$. Regarding $A t t_{v}$ and $A t t_{m f b}$, the red boxes mark the salient regions with the largest and second largest attention weights. Regions are gradually converted to white based on their attention weights where completely white regions mean very low attention weights. Regarding $A t t_{s}$, the text in bold denotes the semantic information that we believe it is important for the generation process. $\quad 86$

6.5 Examples of generated questions by our SMLA and DMLA models with their associated ground-truth questions for yes/no and number answers. . . . . . . . . . . . . . . . . . . . . . . 92 


\section{List of Figures}

1.1 Illustration of the iVQA task. The symbol A stands for a given answer and GQ stands for a generated question by our model. . . . . . . . . 2

1.2 Illustration of traditional CNN and Faster R-CNN on encoding images. In the left figure, the equally sized bounding boxes are corresponding to visual features generated by a traditional CNN (e.g. , ResNet or VGG). In the right figure, the red bounding boxes represent salient objects which are encoded using Faster R-CNN. The labels associated with each bounding box denote the semantic information extracted in the form of "attribute + object". . . . . . . . . . . . . . . . . 4

1.3 Overview of the architectures of our SMLA and DMLA models . . . . 6

2.1 Artificial feed-forward neural network with two hidden layers. The image is taken from [18] . . . . . . . . . . . . . . . . . . . . 9

2.2 The sigmoid function. The image is reproduced from [19] . . . . . . . 10

2.3 The Tanh function. The image is reproduced from [19] . . . . . . . . 11

2.4 Overall architecture of a convolutional neural network. The image is taken from [23] $\ldots \ldots \ldots$. . . . . . . . . . . . . . . . . . . . . 13

2.5 Illustration of a convolution operation with a stride of one and zero padding . . . . . . . . . . . . . . . . . 15 
$2.6 \quad$ Illustration of a pooling operation $2 \times 2$ sliding window and a stride of two . . . . . . . . . . . . . . . . . . . 16

2.7 Example of VGG network with 16 layers. The image is taken from [25] 17

2.8 Comparison between the architecture of the plain network (left) and residual network (right). . . . . . . . . . . . . . . . . . . . 17

2.9 Overall architecture of the vanilla feed-forward neural network (left) and the recurrent neural network (right). . . . . . . . . . . . . . . . . 19

2.10 Illustration of different recurrent neural networks (RNNs) configurations. The image is taken from [26] . . . . . . . . . . . . . . . . . . . 20

2.11 Architectures of Gated Recurrent Network (left) and Long Short Term Memory (right). The image is taken from [31]. . . . . . . . . . . . . 22

2.12 Overall architecture of R-CNN. . . . . . . . . . . . . . . . . . . . . 23

2.13 Overall architecture of Fast R-CNN. . . . . . . . . . . . . . 26

2.14 Overall architecture of Faster R-CNN. . . . . . . . . . . . . . . . . . 27

2.15 Overall architecture of Region Proposal Network (RPN). The image is

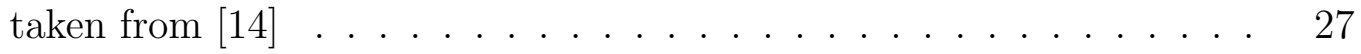

2.16 Example of a Region-of-Interest pooling operation. The image is reproduced from [33]

2.17 The bilienar pooling operation. FC stands for a fully connected layer. The image is reproduced from [36] . . . . . . . . . . . . . . . . . . . . 32

2.18 The MLB operation. The image is reproduced from [36] . . . . . . . . 33

2.19 Illustration of the MFB operation. The image is reproduced from [36] 34

3.1 Overview of the state-of-the-art iVQA model proposed in [1]. In this figure, $M_{t}$ represents the word embedding of the previously generated question word and $q_{t+1}$ denotes the currently generated question word $\quad 40$ 
3.2 Overview of the iVQA model with a variational autoencoder. The figure is reproduced from [2]. In this figure, $z$ is a latent factor, $\mu$ and $\sigma$ represent the mean and variance of $z, M_{t}$ represents the word embedding of the previously generated question word, and $q_{t+1}$ denotes the currently generated question word. . . . . . . . . . . . . . . . . . 42

4.1 The proposed SMLA iVQA model. The model comprises the following components: (1) GRU based answer encoder, which encodes the answer phrase into the semantic embedding space. (2) Faster R-CNN based image encoder, which produces the visual and semantic features of the objects and salient regions. (3) Visual and semantic attention modules, which produces answer attended visual and semantic features. (4) Multi-modal feature fusion (MFB), which fuses the answer cue with the visual and semantic features. (5) MFB attention to attend to the multi-modal fused features (6)Two-layer GRU model, which recurrently generates question words. . . . . . . . . . . . . . . . . . . 48

4.2 Illustration of the Faster R-CNN image encoder module. The detected objects are illustrated in the bottom picture. Each object is localized with a bounding box and annotated with attribute and object labels. 
4.3 The proposed DMLA iVQA model. The model comprises the following components: (1) GRU based answer encoder, which encodes the answer phrase into the semantic embedding space. (2) Faster R-CNN based image encoder, which produces the visual features of the object and salient regions. (3) Multi-modal feature fusion (MFB), which fuses the answer cue with the visual features. (4) Two layers of sequence attentions connected by a dynamic attention mechanism to recurrently generate the question words. . . . . . . . . . . . . . . . . 60

6.1 The architectures of our baseline SMLA model and full SMLA model 82

6.2 Examples of generated questions by our full DMLA model with corresponding dynamic attention visualization (The red bounding boxes localize Regions with the best and second best attention weights. Regions are gradually converted to white based on their attention weights where completely white regions mean very low attention weights). . . 87 


\section{Nomenclature}

\begin{tabular}{|l|l|}
\hline Symbol & Description \\
\hline SMLA & Static Multi-Level Attention model \\
DMLA & Dynamic Multi-Level Attention model \\
RNN & Recurrent Neural Network \\
GRU & Gated Recurrent Unit \\
CNN & Convolutional Neural Network \\
MFB & Multi-model Factorized Bilinear pooling \\
MFB Attention & an attention that attends to the fused features generated using MFB \\
MLB & Multi-modal Low-rank Bilinear pooling \\
$A t t_{s}$ & semantic Attention \\
$A t t_{v}$ & Visual Attention \\
$A t t_{m f b}$ & MFB Attention \\
\hline
\end{tabular}




\section{Chapter 1}

\section{Introduction}

Inverse Visual Question Answering (iVQA) [1, 2] is a new interesting problem in the fields of computer vision and natural language processing. It has emerged recently after the great success of deep learning on the visual question answering (VQA) task [3, 4, 5, 6]. iVQA can be viewed as an extension to the standard task of Visual Question Generation (VQG) [7, 8, 9]. However, different from the VQG task, which automatically generates general questions for a given image, iVQA restricts the question generation to be correlated with a pair of image and answer. Some examples of the iVQA task are shown in Figure 1.1. We can see that for different answers, the intelligent system predicts different corresponding questions that reveal a thorough understanding of fine-grained details of a given image. This illustrates the complexity of the task in contrast to VQG for which a general understanding of an image is sufficient to generate proper questions.

The iVQA task is more important for image understanding than VQA, as a VQA system could predict correct answers merely based on the textual questions without considering the corresponding images [10], and VQA has not yet been able to entirely achieve the main purpose of bridging the understanding gap between textual and 


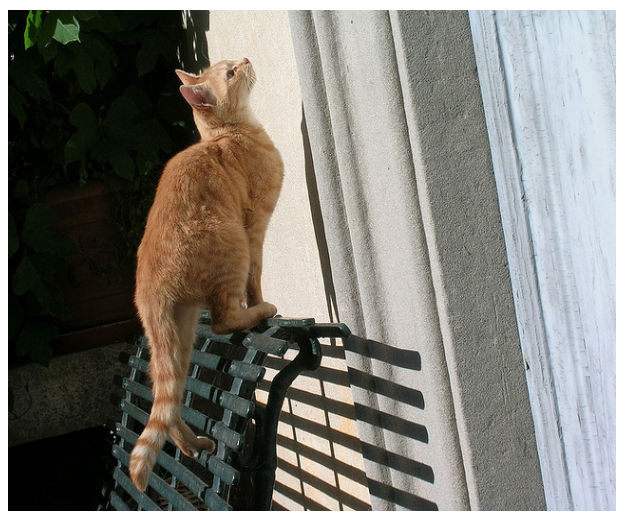

A: bench

A: cat
GQ: What is the cat sitting on?

GQ: What animal is this?

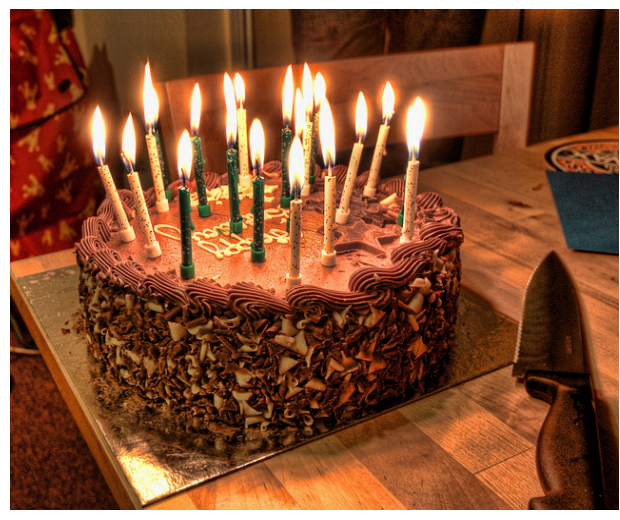

A: chocolate GQ: what flavor is the cake?

A:18 GQ: How many candles are in the photo?

Figure 1.1: Illustration of the iVQA task. The symbol A stands for a given answer and GQ stands for a generated question by our model.

visual modalities. By contrast, an answer phrase is typically much shorter than a question, it is necessary to integrate both visual image understanding and language semantic understanding to yield an effective iVQA system. This makes iVQA a more challenging task, which captures understandings of both image and answer, as well as their complex interactions. It provides a platform for enhancing machine visual and textual understanding. Moreover, iVQA systems can also be used as inexpensive automatic tools to generate questions and create richer datasets for VQA.

As a newly emerged task, iVQA has been studied in a few previous works [1, 2, 11] since its introduction in [1]. The work in [1] has tackled iVQA by enhancing the answer cue with high-level semantic information at the initial step of question generation with the aim of giving the model an overview of the nature of the question to be generated. The question generation model then dynamically attends to important parts of a given image based on the current generation state and the answer cue. The follow-up work in [2] further uses variational auto-encoders to enhance question diversity. In these works, the complex interaction and consistent alignment of the tex- 
tual semantic features with the useful object level visual features, however, remain unexplored. Morever, both iVQA models in [1, 2] extract the visual representation by using a standard deep convolutional neural network (e.g. ResNet [12] or VGG [13]) and can suffer from limitations related to the design of CNNs [4]. A typical CNN model generates visual features composed of slices (subfeatures) generated using a fixed size receptive field and hence the slices are correlated with equally sized parts in an input image. However, these parts do not consistently represent useful objects [4] , as shown in Figure 1.2. This raises problems for applying attention mechanisms in iVQA models, as the model may select some regions that are not completely aligned with the desired objects while irrelevant data may be included in the attention feature and negatively affect the prediction decision. This is illustrated in Figure 1.2(left), where there are four regions that bound the horse object and some of the regions include noisy data (e.g. trees) which may distract the used model and consequently generate inaccurate questions. To overcome this problem, we adopt a bottom-up attention mechanism [4], based on Faster R-CNN [14], to extract visual features at the level of objects and salient regions as shown in Figure 1.2 (right). The effect of the Faster R-CNN method was validated by achieving the state-of-the-art results on the object detection task. By using Faster-CNN for iVQA, we develop two novel models, a Static Multi-Level Attention (SMLA) model and a Dynamic Multi-Level Attention (DMLA) model as shown in Figure 1.3. In the SMLA model, we focus on investigating different attention mechanisms to capture the image and answer relationship at different levels of knowledge. In the DMLA model, we explore the use of the attention mechanism as an alternative to the conventional sequence learning techniques such as the recurrent neural network $(\mathrm{RNN})$ and its variants.

SMLA Model. We have found that a considerable number of the ground-truth 

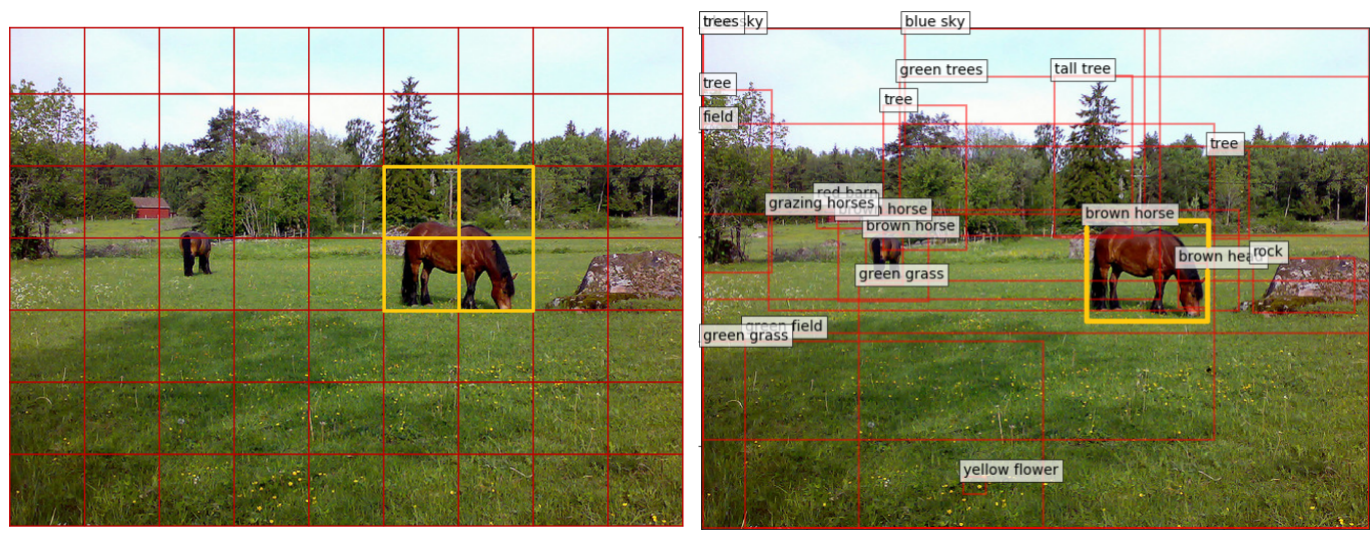

Figure 1.2: Illustration of traditional CNN and Faster R-CNN on encoding images. In the left figure, the equally sized bounding boxes are corresponding to visual features generated by a traditional CNN (e.g. , ResNet or VGG). In the right figure, the red bounding boxes represent salient objects which are encoded using Faster R-CNN. The labels associated with each bounding box denote the semantic information extracted in the form of "attribute + object".

questions in the VQA dataset [15] involve asking about a specific object in a given image (e.g. "How many birds are there?", or "What color is the bird?"). To generate these kinds of questions, it is sufficient to use a static attention that directly focuses on a specific object corresponding to a given answer. However, for more complicated questions such as "What is the man wearing on his head?", an iVQA model should focus on many objects (e.g. , the man, his head) while generating questions. In our SMLA model, we handle these cases by using a novel static multi-level attention mechanism to generate either complicated or simple questions based on given answers. The new mechanism incorporates three answer-based static attention modules at both the visual and semantic levels. The first visual attention is similar to that used in [11]. The second Multi-Factorized Bilinear (MFB) pooling-based attention module determines the attention weights by considering both the semantic and visual cues based on a given answer. It leverages the object labels generated by Faster R-CNN to enhance the corresponding visual features and can assist the model to 
bridge the semantic gap [5] between the given image and answer. The last semantic attention module, used in our SMLA model, is added as an auxiliary attention to help the model leverage those semantic concepts corresponding to a given answer. Integrating the three attention mechanisms can help our SMLA model to attend to the relevant object-level parts of a given image and at different levels to generate correct and corresponding simple and complicated questions.

DMLA Model. Generating complicated questions such as "What is the man behind the chair wearing on his head?" requires an iVQA model to attend to different objects to generate accurate questions. For such questions, it is helpful to use an attention module that dynamically attends to important visual objects, given the answer and current partially generated question. In this context, we develop a novel DMLA model as shown in Figure 1.3(right). In addition to adopting the object-based visual features to represent images, we investigate using attention mechanisms to replace recurrent neural networks for question encoding and decoding. Consequently, our DMLA model is not only beneficial to dynamically focus on salient regions in a given image as in [1], but also to focus on the important previously generated question keywords alongside the answer for the current generation state. Our study shows that even without leveraging the additional knowledge represented by semantic concepts, our DMLA model outperforms previous iVQA models in many benchmarks and has comparable results to our SMLA model.

\section{Main Contributions}

In this thesis, we have made the following contributions:

- We develop a novel static multi-level attention (SMLA) model for inverse visual question answering (iVQA), which integrates three attention modules to cap- 


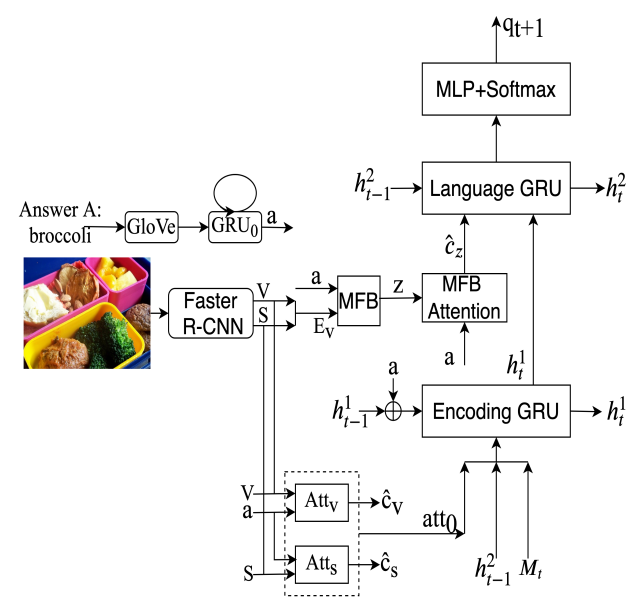

The SMLA model

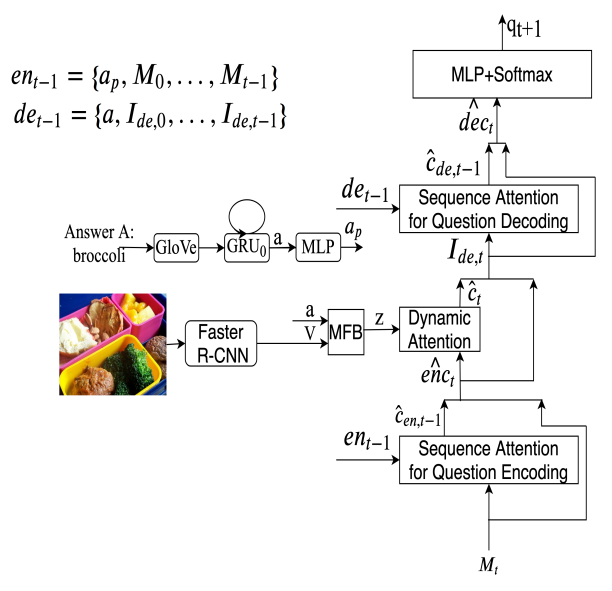

The DMLA model

Figure 1.3: Overview of the architectures of our SMLA and DMLA models

ture complex image-answer relationships at different levels to induce consistent regional textual and visual feature alignment with the answer cue for question generation.

- We develop a novel dynamic multi-level attention (DMLA) model and investigate the use of dynamic attention mechanisms as a powerful alternative to the conventional recurrent neural networks for question encoding and decoding.

- We conduct experiments on the standard VQA v1 dataset to investigate the empirical performance of the proposed models by comparing them with the state-of-the-art iVQA methods [1, 2]. Our proposed models achieve the stateof-the-art performance. 


\section{Chapter 2}

\section{Background}

Deep learning has become the state-of-the-art for many complex tasks in computer vision and natural language processing. It has strong capabilities to learn complex high and low-level hierarchical data representations. Although the fundamentals of deep learning techniques like neural networks and recurrent neural networks were developed in the second half of the previous century, the feasible usage of deep learning started in 2012 [16]. This breakthrough is due to many factors including the availability of powerful computational resources and large datasets. Nowadays, deep learning algorithms are the most useful machine learning algorithms for the tasks of Visual Question Answering (VQA), image captioning and Visual Question Generation (VQG).

In this chapter, we present the main deep learning concepts. Starting from artificial neural networks in section 2.1 and its variants represented by convolutional neural networks in section 2.2 and recurrent neural networks in section 2.3. In section 2.4 . we describe the state-of-the-art techniques for object detection. Finally, in section 2.5 , we introduce the most popular approaches used for visual and textual fusion. 


\subsection{Artificial Neural Networks}

An Artificial Neural Network (ANN) was developed to artificially mimic our nervous systems. ANNs constitute the basis for deep learning algorithms. The purpose of any neural network is to learn a specific function from input data. Therefore, neural networks have learnable parameters called weights which can be modified by training a neural network to learn a target function. The training can be either supervised or unsupervised. Regarding the supervised training, both input and output data are available. The role of neural networks is to learn a function mapping the inputs into outputs. This can be achieved by using a training algorithm like back-propagation with an optimizer like Adam [17]. On the other hand, with unsupervised learning, only the input data are available. Therefore, the task of neural networks would be to split similar input data into clusters. However, this method is not commonly used with state-of-the-art neural networks, which typically adopt supervised learning for training.

\subsubsection{Feed-forward Neural Networks}

The feed-forward neural network is the standard artificial neural network. It is composed of multiple neurons at various layers, where every neuron from the layer $l_{i}$ is linked to all neurons in the layer $l_{i+1}$ as shown in Figure 2.1. A standard neuron has many inputs with their associated weights. The output $O$ of such a neuron can be computed as follows:

$$
O=f\left(\sum_{i=0}^{n} w_{i} x_{i}\right)
$$

where $x_{0}$ is the bias term, $x_{1}, x_{2}, \ldots, x_{n}$ are the inputs, $w_{i}$ represents weights to be learned, and $f$ is an activation function (e.g. tanh, Softmax). 


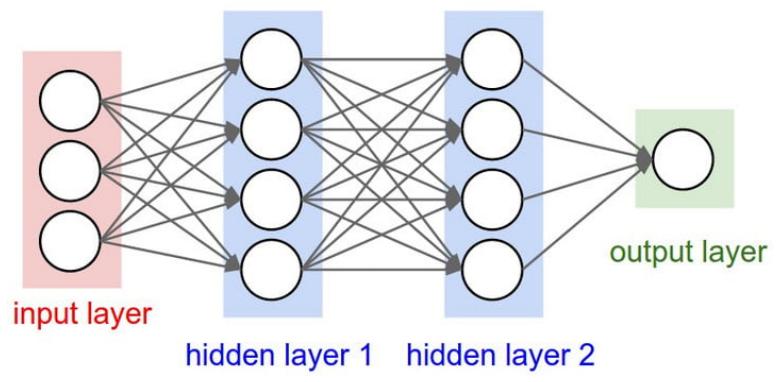

Figure 2.1: Artificial feed-forward neural network with two hidden layers. The image is taken from [18]

As shown in Figure 2.1, the artificial feed-forward neural network consists of three main layers as follows:

- Input Layer: the first layer, where input data should be applied.

- Hidden Layer: the intermediate layer that processes data coming from the input layer, learns its modelling, and delivers information to the output layer (or next hidden layer in case the neural network has more than one hidden layer).

- Output Layer: the final layer, where the activation function is applied. It produces the results (the output) of the model learned by the applied neural network.

\subsubsection{Activation Function}

It is standard to use activation functions in artificial neural networks. The primary role of these functions is to integrate non-linearity into neural networks. To explain, a standard neuron without an activation function, is just a linear function. The combination of all the neurons in a neural network also results in a linear function. As this may not be beneficial to learn complex tasks, we use non-linear activation 
functions to enhance the ability of neural networks to learn high order functions. Below, we present four of the most useful functions.

1. Sigmoid Function (Logistic Function). It is a differentiable activation function that maps the input values into values in the range $[0,1]$. It is common to use the sigmoid function at the last layer for binary classification. The sigmoid function output is shown in Figure 2.2 and is defined as follows:

$$
f(z)=\frac{1}{\left(1+e^{-z}\right)}
$$

where $z$ represents the input to the sigmoid function.

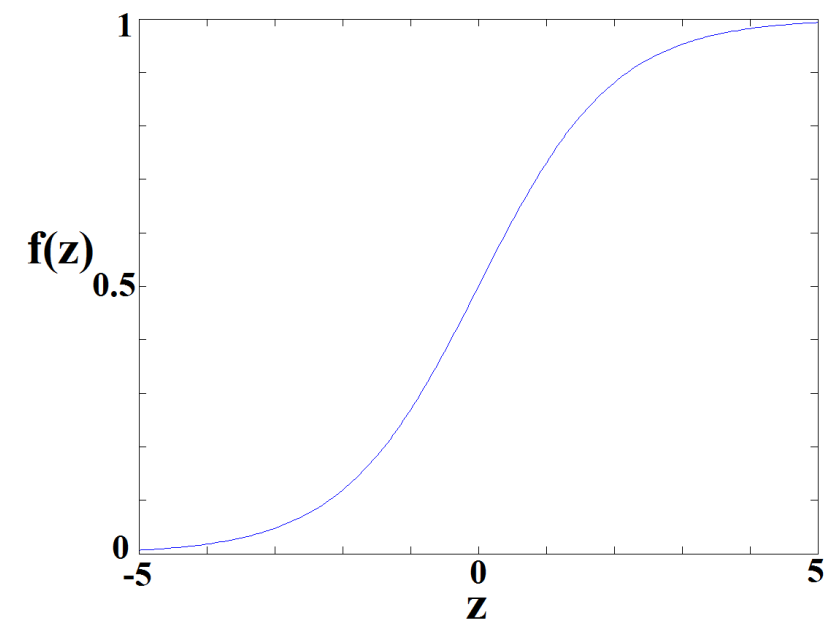

Figure 2.2: The sigmoid function. The image is reproduced from [19]

2. Tanh Function. It is similar to the sigmoid function with some improvements. It maps the input values to values between $(-1$ and +1$)$. The Tanh function could be more representative than the sigmoid function as it has the features to transfer positive inputs into +1 and small values into 0 and importantly negative values into -1 as shown in Figure 2.3. The Tanh function is more popular than the sigmoid function. It is mostly utilized in the hidden layers for 
complex function approximation. Mathematically, the Tanh function is defined as follows:

$$
f(z)=\frac{e^{z}-e^{-z}}{e^{z}+e^{-z}}
$$

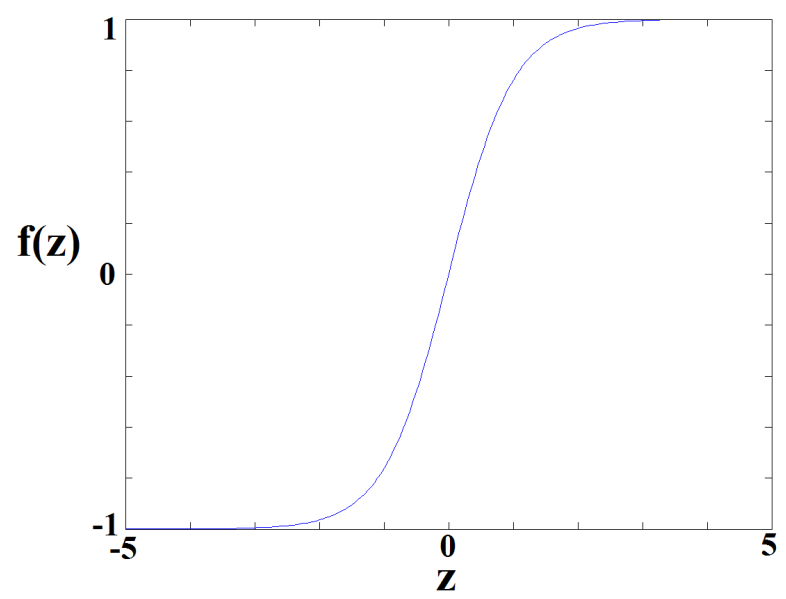

Figure 2.3: The Tanh function. The image is reproduced from [19]

3. ReLU Function. ReLU stands for Rectified Linear Unit. It is widely used in most deep learning algorithms. It is one of the key components of state-ofthe-art deep convolutional neural network models [12, 13]. The ReLU function keeps non-negative input values and maps negative values to 0 . Mathematically, it is defined as follows:

$$
f(z)=\max (0, z) .
$$

4. Softmax Function. It is typically used for multi-class classification. It is added at the last layer of a neural network to determine the probability distribution of the output over a set of classes. Mathematically, the softmax function 
is defined as follows:

$$
f(z)=\frac{e^{z_{i}}}{\sum_{j=1}^{K} e^{z_{j}}}
$$

\subsubsection{Training Neural Networks}

The most useful supervised learning algorithm to train neural networks is backpropagation [20] with an optimizer like gradient descent or its variants such as Adam [17]. With the back-propagation algorithm, the input data are feed-forwarded into a neural network from the input to the output layer. Following this, a predefined loss function (e.g. , a squared-error function) is used to determine the error between the predicted outputs $\hat{y}$ and the required outputs $y$ (labels). Then, this error is propagated back through the neural network, from the output to the input layer, to modify its weights and biases by applying an optimizer like gradient descent. The previous process repeats many times until a specific stopping criterion is met (e.g. the error is smaller than a predefined threshold).

\subsection{Convolutional Neural Networks}

A Convolutional Neural Network (CNN) is one of the artificial neural network variants. It has demonstrated great performance with many computer vision tasks like image classification [12, 13] and object detection [14, 21, 22]. It is currently the state-of-the-art visual feature extractor for deep learning tasks that combines both computer vision and natural language processing such as image captioning, visual question answering and visual question generation. Since its introduction, the architecture of CNNs has shown a significant evolution, especially related to their depth and width. However, as shown in Figure 2.4, each CNN model includes the following main layers: 


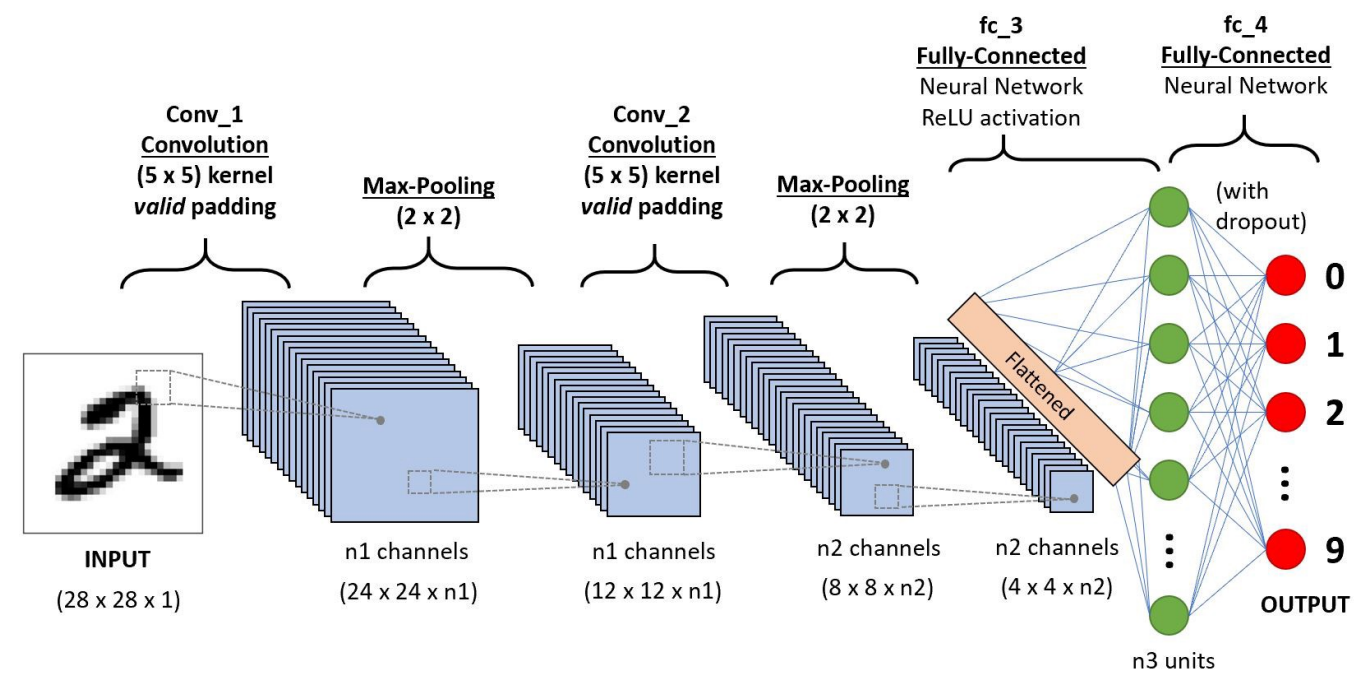

Figure 2.4: Overall architecture of a convolutional neural network. The image is taken from 23 .

1. Convolutional layer. This is the main module of any CNN. It is usually the first layer where images are applied. This layer uses a set of convolution kernels (filters) on an image to extract its features. Figure 2.5 clarifies how a feature map is generated. An element-wise multiplication is performed between the convolution filter and a part (e.g. top-left) of an input image. The results are summed to obtain a scalar representing the output of the first convolution operation. Similarly, the filter slides over all parts of an input image and applies the convolution operations to finally obtain a feature map that represents the given image. To enrich this representation, it is common to use various filters, where each filter is responsible for capturing a specific aspect of the given image (e.g. , lines, edges, colours, etc.). The applied filters produce associated feature maps that are stacked altogether to form the final 3-dimensional image feature. As the size of the applied filters is fixed within this convolutional layer, the fea- 
ture slices of the generated 3-dimensional feature map correspond to equal-sized parts in the given image.

The size of the generated 3-dimensional image feature varies from one convolutional layer to another based on the following predefined hyper-parameters [24]:

- Receptive field: represents the convolution filter size. It should be smaller than the size of an input image.

- Depth: represents the number of filters in a convolutional layer.

- Stride: controls the number of steps that the sliding convolution filter should move at each new convolution operation.

- Padding: a zero-padding is added around an input image. The most useful application of this method is to obtain an image feature map with the same spatial size as the input image.

Specifically, given an input image $I \in R^{H_{i n} \times W_{i n} \times D_{i n}}, N$ receptive fields of size $K$, a padding zero $P$, and a stride $S$, then the size of the 3 -dimensional image feature $I_{\text {out }} \in R^{H_{\text {out }} \times W_{\text {out }} \times D_{\text {out }}}$ is given as follows [24]:

$$
H_{\text {out }}=\frac{H_{\text {in }}-K+2 P}{S}+1, \quad W_{\text {out }}=\frac{W_{\text {in }}-K+2 P}{S}+1, \quad D_{\text {out }}=N
$$

2. Non-linearity Layer. After extracting an image feature using a convolutional layer, it is common to apply a non-linear function such as ReLU (Rectified Linear Unit) which only keeps non-negative values and suppresses the negative ones. 


\begin{tabular}{|l|l|l|l|l|}
\hline 1 & 0 & 1 & 0 & 0 \\
\hline 0 & 1 & 0 & 1 & 0 \\
\hline 0 & 0 & 0 & 0 & 1 \\
\hline 0 & 0 & 0 & 0 & 1 \\
\hline 1 & 0 & 0 & 0 & 1 \\
\hline
\end{tabular}

Input image

\begin{tabular}{|l|l|l|}
\hline 0 & 0 & 1 \\
\hline 0 & 0 & 1 \\
\hline 0 & 0 & 1 \\
\hline
\end{tabular}

Filter

\begin{tabular}{|l|l|l|}
\hline 1 & 1 & 1 \\
\hline 0 & 1 & 2 \\
\hline 0 & 0 & 3 \\
\hline
\end{tabular}

Feature map

Figure 2.5: Illustration of a convolution operation with a stride of one and zero padding .

3. Pooling Layer. It performs a downsampling operation on an image feature. It can be added after the convolutional layer and it is very useful for many reasons such as dimensionality reduction, computational efficiency, decreasing overfitting and enhancing CNN model generalization. The most common pooling operations are the max pooling, average pooling, and sum pooling.

Figure 2.6 shows how a pooling operation can be implemented. Specifically, a window of size $m \times m$ is applied spatially on a part of an image feature. At that point, a pooling operation is performed to output a scalar. Similarly, the window performs pooling operations on the other parts of the image feature to finally obtain the downsampled version of the image feature. This feature should maintain the same depth as the applied image feature, but it is spatially smaller. After that, the generated feature can be either fed into another convolutional layer to enrich image features at a different hierarchy or into a fully connected layer for classification.

4. Fully connected layer. Each neuron in the fully connected layer is connected with all neurons in the preceding layer. It is mostly applied at the end of a CNN to classify images. It is common to use several consecutive fully connected layers, where the output size of the last layer is equal to the number of required 


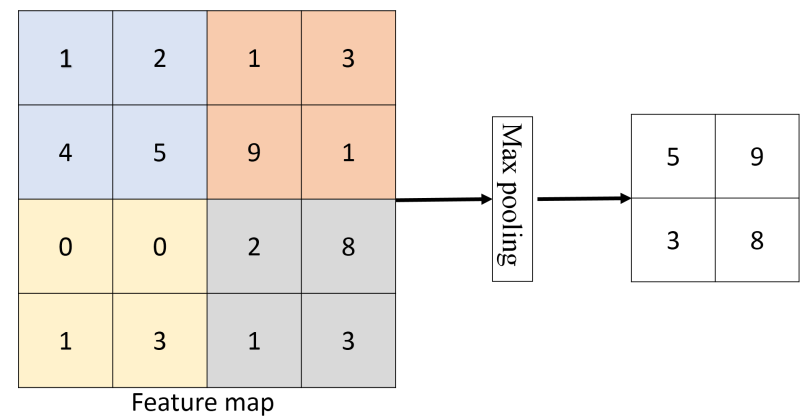

Figure 2.6: Illustration of a pooling operation $2 \times 2$ sliding window and a stride of two

classes. A Softmax function is usually used as a final layer in a CNN to generate a probability distribution over the given classes. Consequently, images are classified by taking the output with the highest predicted probability.

State-of-the-art Convolutional Neural Networks. After the great success of CNNs in the tasks of image representation and object detection, much research has been conducted to improve their performance. In this section, we present two of the most outstanding state-of-the-art CNNs, namely, VGG [13] and residual networks $[12$.

VGG. In 2014, Simonyan et al. developed a deep CNN model called VGG [13]. Through their model, they show how important it is to increase the depth of a CNN for better performance. They presented various models with 16 to 19 convolutional and fully connected layers. For each convolutional layer, a receptive field of size $3 \times 3$ with a stride of 1 pixel is used. The pooling operation is implemented by using a max pooling function and a sliding window sized $2 \times 2$ with a stride of 2 pixels. At the end of the VGG network, three fully connected layers are inserted and followed by a Softmax layer to predict the output probability distribution over a set of 1000 classes. 


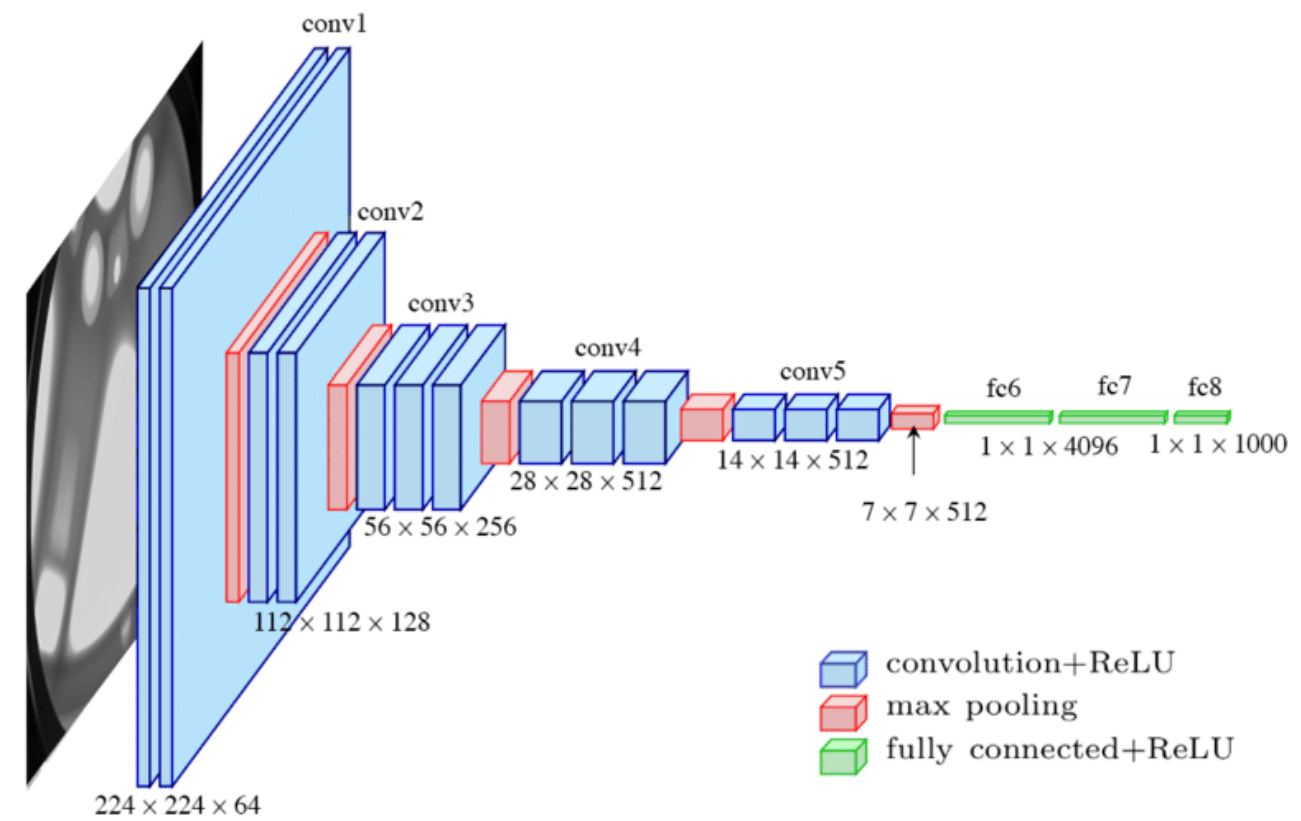

Figure 2.7: Example of VGG network with 16 layers. The image is taken from [25]

Residual Networks. The significant performance gain of VGG motivates re-
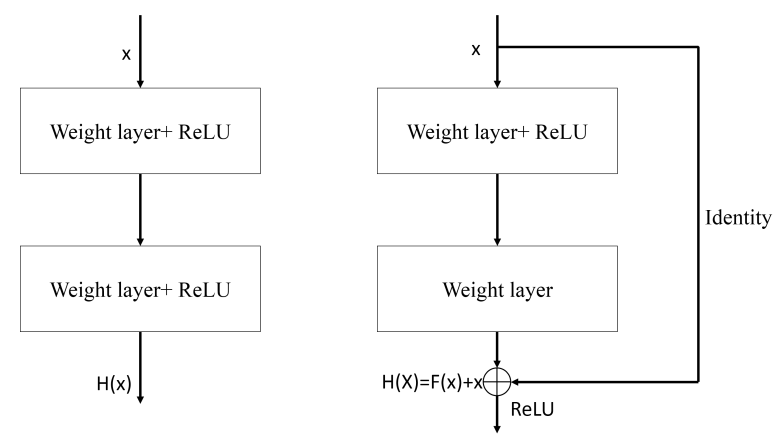

Figure 2.8: Comparison between the architecture of the plain network (left) and residual network (right).

searchers to develop deeper CNNs. In 2015, He et al. [12] introduced the deep residual networks with up to 152 convolutional and fully connected layers. However, developing such a deep CNN required the authors to introduce the residual block to 
handle problems related to the gradient vanishing and accuracy degradation caused by increasing the network's depth.

In Figure 2.8, we can see the difference between the residual network block and the plain network block. Regarding the residual network block, a skip connection is used to directly add the input $x$ to the residual function $F(x)$ to obtain the target mapping $H(x)=F(x)+x$. However, this is different in the plain network block where a direct mapping between $x$ and $H(x)$ is learned. In the former case, the network only learns the residual function $F(x)$ which might differ slightly from the input, while in the latter case, the plain block must learn an entirely new function $H(x)$. This can be clarified in case of optimal identity mapping, where the residual block only needs to force the residual function $F(x)$ to approach zero which is much easier than learning the identity function $H(x)=x$ by using a plain CNN.

Residual networks are now the state-of-the-art convolutional neural networks. The authors examine the effects of network depth by applying a different number of layers (e.g. , 50, 101, 152 and 1202). Their 152-layer model was the best and surprisingly better than the deeper 1202-layer model. This reveals the need for more research to thoroughly understand the CNNs' depth and performance relationship.

\subsection{Recurrent Neural Networks}

A Recurrent Neural Network (RNN) is another variant of artificial neural networks. It is mainly used with sequence prediction tasks such as machine translation, question answering, and visual question generation. Its capability of learning long-term dependencies makes it the best choice for an effective sequence representation.

Vanilla feed-forward neural networks, as shown in Figure 2.9 (left) takes an input and feed forward it in one direction to the output. In contrast, recurrent neural networks 

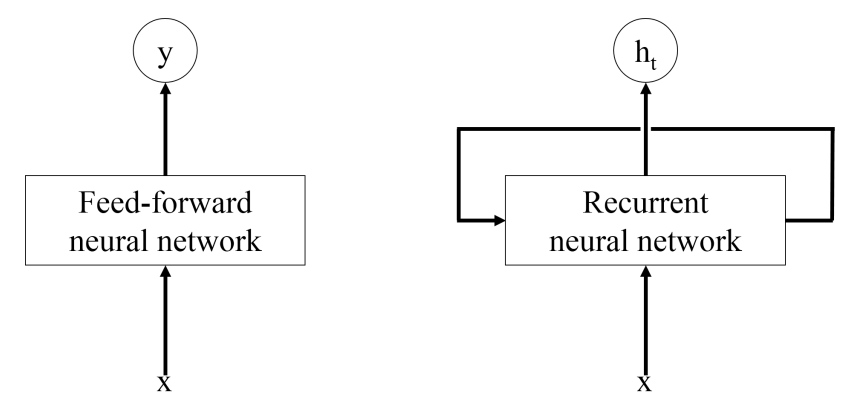

Figure 2.9: Overall architecture of the vanilla feed-forward neural network (left) and the recurrent neural network (right).

have feedback loops which work like a memory that helps RNN to predict an output based not only on the currently applied input, but also on the knowledge learned from previous inputs.

As shown in Figure 2.10, RNNs can take one of the following architectures:

- One-to-many: an RNN accepts one input and generates a sequence of outputs.

- Many-to-One: an RNN accepts a sequence of inputs and generates one output.

This model is widely adopted in the task of visual question answering.

- Many-to-many: an RNN accepts a sequence of inputs and generates a sequence of outputs. This model is used with image captioning, visual question generation, and inverse visual question answering.

The conventional RNNs have provided considerable improvements for sequence learning problems. However, their great performance has been limited to learning short and simple sequences. In other words, RNNs have been unsuccessful with complex long sequence learning tasks (e.g. dialogue learning). This is principally because of the vanishing gradient problem [27] which makes gradients too small as they have to go through a long consecutive chain of RNNs. Consequently, the learning process may stop or become extremely slow in finding the optimal solution. To explain, when 

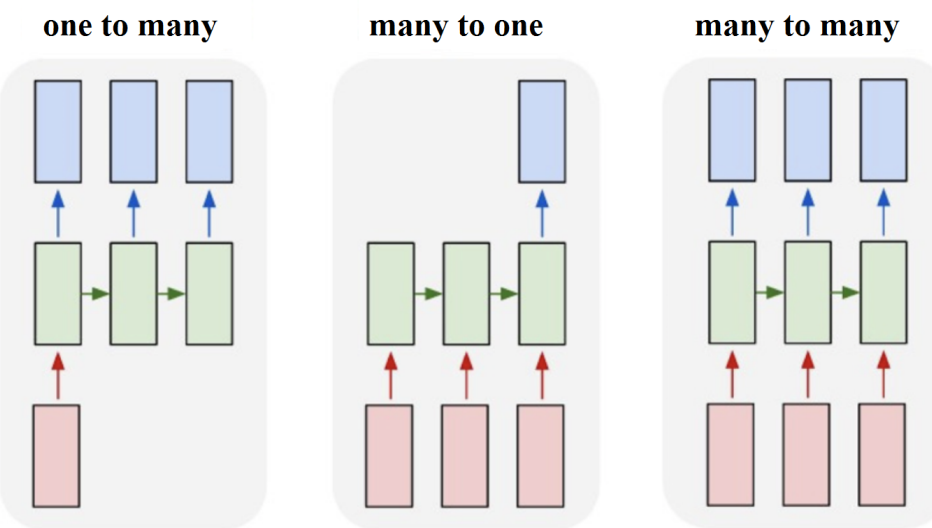

Figure 2.10: Illustration of different recurrent neural networks (RNNs) configurations. The image is taken from [26]

the gradient is close to zero, an RNN may think that the optimal solution has been determined and no weight update is required, and therefore stop learning. To address this problem, many variants on RNNs have been developed, namely Long Short Term Memory (LSTM) [28] or more recently, Gated Recurrent Unit (GRU) [29].

\subsubsection{Gated Recurrent Units (GRUs)}

GRU was recently developed by Cho et al. [29] in 2014. It was initially designed for the task of machine translation. This special kind of RNN was derived from LSTM. However, the architecture of GRU is simpler than that of LSTM as shown in Figure 2.11 (left). This simplicity helped in reducing the number of parameters needed to build the gated recurrent unit. Therefore, GRUs are promising and may exceed LSTMs with regards to computational efficiency and parameter refinements [30].

GRU Architecture. A GRU unit [29, 30] has one output and two inputs; the first input is allocated for data $x$ and the second input takes the output of a previous GRU, namely hidden state $h_{t-1}$. The hidden state is responsible for carrying infor- 
mation about previous dependencies and injecting them into the current GRU. This information flow is controlled through two gates; the reset and update gates. The reset gate $r$ decides the amount of previous information that the current unit should drop, while the update gate $z$ decides how important the previous state is for the current unit. The reset and update gates of a GRU, at step $t$, can be computed as follows:

$$
\begin{aligned}
& r_{t}=\sigma\left(W_{r} x_{t}+U_{r} h_{t-1}\right) \\
& z_{t}=\sigma\left(W_{z} x_{t}+U_{z} h_{t-1}\right),
\end{aligned}
$$

where $W_{r}, U_{r}, W_{z}$, and $U_{z}$ are weights to be learned, and $\sigma$ is the sigmoid activation function.

Then, the final hidden state $h_{t}$ of the current GRU, which determines what information to keep for current and next time step, is computed as follows:

$$
\tilde{h}_{t}=\tanh \left(W_{h} x_{t}+\left(r_{t} \odot U_{h} h_{t-1}\right)\right), \quad h_{t}=z_{t} h_{t-1}+\left(1-z_{t}\right) \tilde{h}_{t},
$$

where $W_{h}$, and $U_{h}$ are weights to be learned, and $\odot$ is an element-wise multiplication.

GRU vs LSTM. Both GRUs and LSTMs have shown great performance with sequence learning tasks. However, in some tasks, LSTMs may slightly outperform GRUs or vice versa [30]. Therefore, it is not a straightforward task to judge which one is better.

In terms of the architecture, Figure 2.11 shows that both GRU and LSTM have similar architectures. However, there are some differences. For example, LSTM has three 
internal gates (forget, input, and output.) instead of two in GRU. In addition, LSTM has two outputs; the hidden state $h$ and the cell state $c$, compared to only one output in GRU. Accordingly, GRU has fewer parameters compared to LSTM and therefore GRU is better in terms of the computational efficiency.
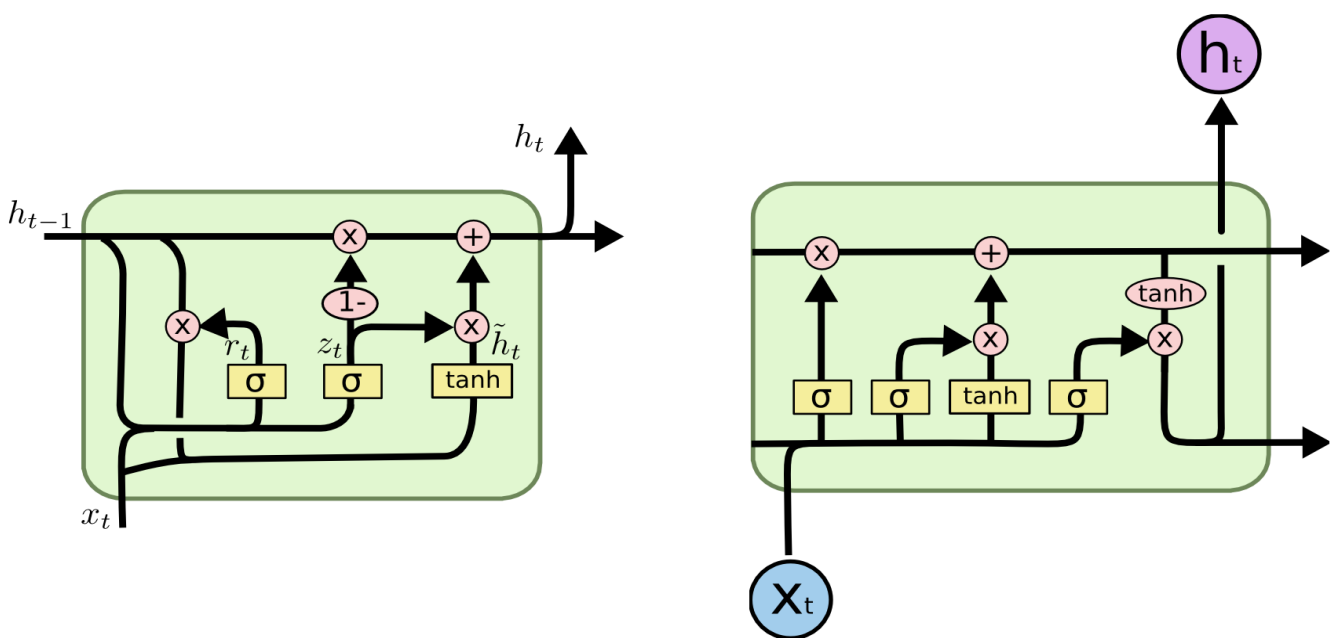

Figure 2.11: Architectures of Gated Recurrent Network (left) and Long Short Term Memory (right). The image is taken from [31].

\subsection{Object Detection}

Standard CNN models like ResNet [12] and VGG [13] take an input image and generate labels and probabilities of the recognized objects in the given image. However, they lack the ability to detect the locations of the recognized objects. To address this issue, the object detection task emerged to not only classify the recognized objects, but also to generate bounding boxes to localize these objects. Currently, the prominent object detection algorithm is the Region Based Convolutional Neural Network (R-CNN) algorithm [22] and its modified variants, namely Fast R-CNN and Faster R-CNN [14, 21]. These techniques can be utilized to extract object-based visual features with the associated textual labels (semantic information), which can then 
be applied to our model. Our model adopts the state-of-the-art Faster R-CNN [14] algorithm. However, it is important to understand the preceding R-CNN and Fast R-CNN algorithms as we describe them below.

\subsubsection{R-CNN}

A Region-Based Convolutional Neural Network (R-CNN) [22] was recently introduced to solve the challenging problems of object localization and recognition. Overall, RCNN takes a given image as input and then localizes the salient objects and generates the corresponding labels and class probabilities. For each of the extracted objects, R-CNN generates a bounding box with four components; $P=\left(P_{x}, P_{y}, P_{w}, P h\right)$, where $P_{x}$ and $P_{y}$ represent the center coordinates, and $P_{w}$ and $P_{h}$ are the width and height of the given box respectively. This gives the exact location and the size of the extracted object compared to other objects in the given image.

The R-CNN workflow is shown in Figure 2.12 and it consists of three main steps:

1. Generating Region proposals.

2. Visual features extraction.

3. Regions classification.

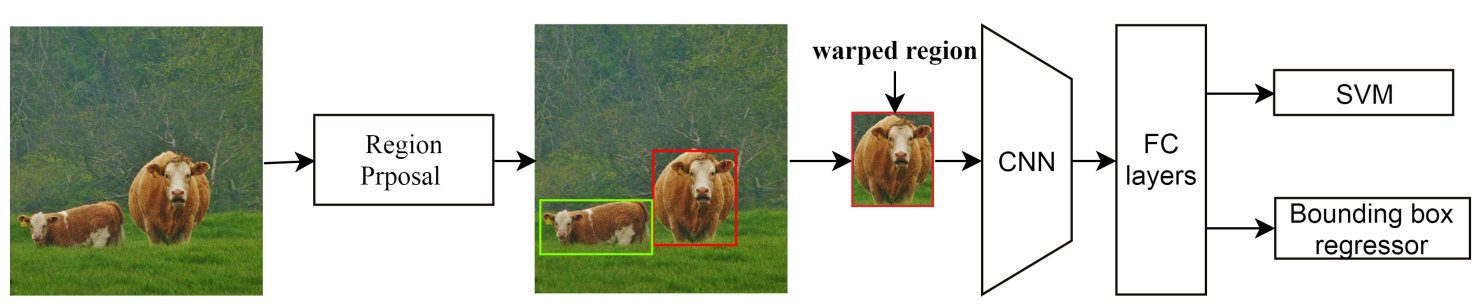

Figure 2.12: Overall architecture of R-CNN.

Region Proposals. R-CNN adopts the Selective Search algorithm [32] to extract region proposals from a given image. The Selective Search first applies image segmen- 
tation to partition a given image into a set of segments. Following this, the algorithm iteratively groups similar neighbouring segments and merges them into one bigger region using different similarity criteria (e.g. colour, texture, and size). This bottom-up grouping process repeats until extracting 2000 salient regions that represent the list of region proposals.

Visual Features Extraction. After obtaining the region proposals, R-CNN uses a deep CNN (e.g. , VGG [13]) to generate image features for each of the regions. However, before applying the region proposals into the $\mathrm{CNN}$, each region is reshaped to have a fixed dimension. This transformation is necessary to assure each region is compatible with the required input of the selected CNN architecture. The regions are then applied to the CNN, which outputs a 4096-dimensional visual feature for each of the extracted regions.

Regions Classification. In this step, R-CNN takes the visual features of the region proposals and then determines the regions that represent meaningful objects and classifies them using multiple class-based Support Vector Machine (SVM) classifiers. Then, for each class, R-CNN removes the overlapping regions if their IntersectionOver-Union (IOU) values are higher than a predefined threshold. In the end, we should have predicted bounding boxes localizing the salient objects in the given image and a visual feature vector for each of the objects. The predicted bounding boxes are refined by comparing them with the ground truth bounding boxes using regression. 


\subsubsection{Fast R-CNN}

R-CNN significantly improved the accuracy and efficiency of object detection. However, it still experiences some critical problems that prevent its effective use with large datasets. The major drawback that considerably affects the performance of R-CNN is that each of the selected object proposals should be feed-forwarded independently through a deep CNN. Consequently, $2000 \mathrm{CNN}$ operations are needed to generate the corresponding visual features. Moreover, the training of R-CNN involves three separate modules; the CNN-based feature extractor, the SVM classifiers, and finally regressors for bounding boxes refinements. To overcome these problems, Girshick proposed his modified version of R-CNN, which is called Fast R-CNN [21].

Fast R-CNN is faster than R-CNN at both the training and testing phase. Many optimization steps were implemented to achieve this improvement. As shown in Figure 2.13, with the Fast R-CNN algorithm, the whole image is forward propagated through a CNN, which generates a single global feature map for the given image. Simultane-

ously, the Selective Search algorithm extracts the region proposals. Subsequently, a Region-Of-Interest (ROI) pooling layer is applied on the feature maps corresponding to the object proposals, to generate equally-sized visual features. Each visual feature is then applied to fully connected layers that then branch out into a softmax layer for classification and a bounding box regressor for localization refinement. Fast R-CNN is trained in an end-to-end manner, which makes it 9 times faster than R-CNN.

\subsubsection{Faster R-CNN}

Faster R-CNN [14] is a state-of-the-art object detection algorithm that was developed to solve the drawbacks of Fast R-CNN. Although Fast R-CNN made great 


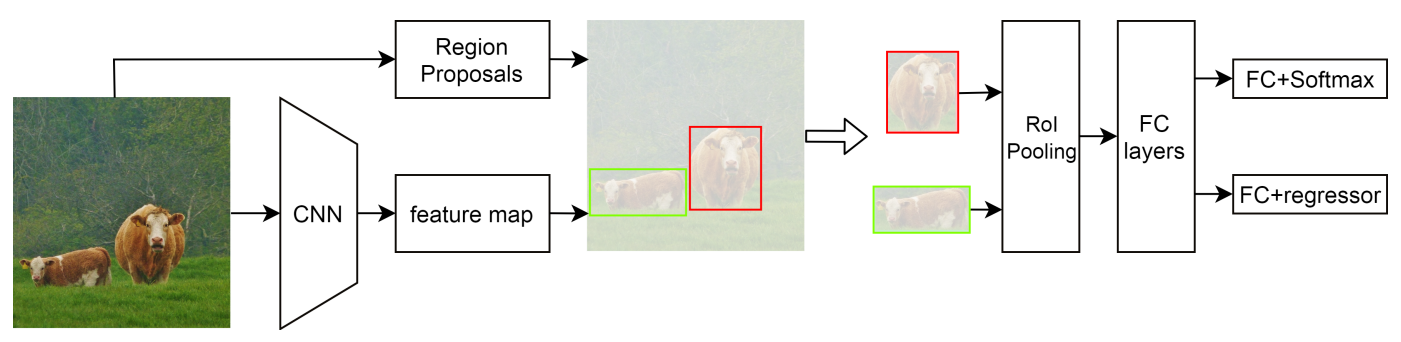

Figure 2.13: Overall architecture of Fast R-CNN.

improvements over R-CNN, extracting the region proposals is still a computational bottleneck. Similar to R-CNN, Fast R-CNN adopts Selective Search algorithm 32] to generate the regions proposals. This algorithm needs around 2 seconds to generate region proposals for each image. In order to improve this, Faster R-CNN extracts the region proposals using a new technique called the region proposal network (RPN). This method reduces the running time to generate the region proposals from 2 seconds, using Selective Search, to around 10ms. Below, we present the architecture of Faster R-CNN and the principal approaches to train it.

Faster R-CNN Architecture. The architecture of Faster R-CNN is shown in Figure 2.14. Overall, Faster R-CNN first applies a CNN (e.g. ResNet [12]) on a given image to extract corresponding feature maps. Following this, Faster R-CNN extracts region proposals using RPN. Subsequently, the Region-of-Interest (RoI) pooling layer unifies the dimensions of the extracted object proposals, which are then fed into fully connected layers that branch out into a softmax layer for classification, and a bounding box regressor for refining localization. Detailed information about the key components of Faster R-CNN is provided in the following subsections.

(A) Region Proposal Network Architecture. Unlike Fast R-CNN which extracts region proposals directly from a given image using Selective Search, Faster R-CNN first extracts feature maps using a CNN and then creates object pro- 


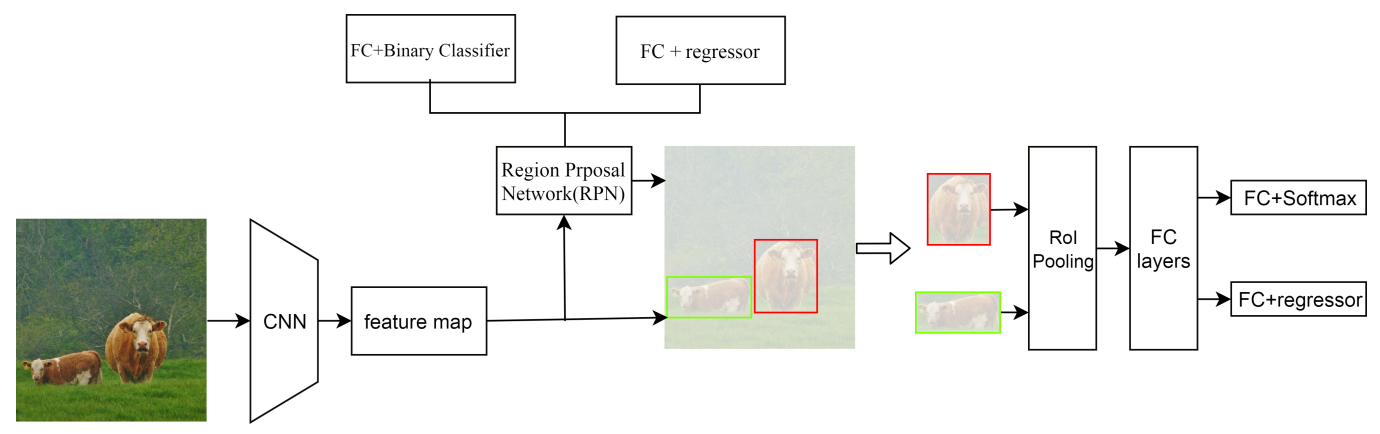

Figure 2.14: Overall architecture of Faster R-CNN.

posals from these maps using RPN. The workflow of RPN is shown in Figure 2.15. Firstly, RPN uses a sliding window of small size $(e . g .3 \times 3)$ and applies

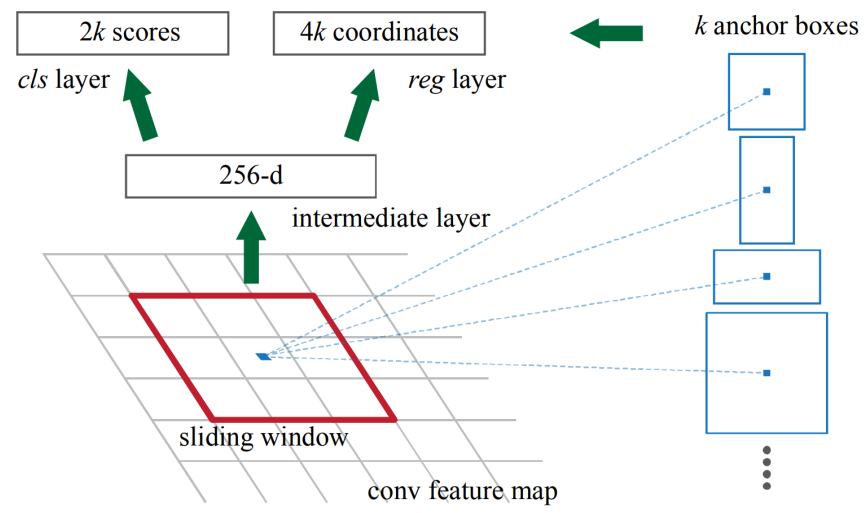

Figure 2.15: Overall architecture of Region Proposal Network (RPN). The image is taken from [14]

it to the convolutional feature map to check for the existence of any potential objects. To ensure that all objects are captured at the given position of the sliding window, RPN applies $k$ different anchors at the center of the sliding window. An anchor is basically a rectangular box that helps Faster R-CNN to scan objects at various scales and aspect ratios. Specifically, 9 different anchors are applied each time the sliding window changes its position. For each anchor, RPN generates a fixed-size feature map and then applies it into two parallel fully connected layers. The first layer is a binary classification layer that pre- 
dicts the objectness score, which measures how likely the corresponding anchor represents a meaningful object. The second layer is a regression layer that refines the coordinates of the applied anchor based on the ground truth object location. After sliding the window over every position in the feature map, RPN would output the required region proposals (or object proposals).

(B) Region Proposal Networks Training. The loss function that Faster RCNN uses to train RPN is a combination of two components- the binary classification loss and regression loss. The binary classification loss classifies anchors based on their probabilities to represent objects. To prepare the anchors for the training phase, we set a class label (e.g. 0 or 1) for each anchor by comparing it with ground-truth boxes. Then, only the anchors that ovralap with ground-truth boxes with Intersection-Over-Union (IOU) ratios greater than a pre-specified threshold, are assigned as class 1 (object). With regards to the regression loss, it refines the locations of the anchors classified as potential objects, by comparing them with their corresponding ground truth bounding boxes. Based on the previous description, the loss function of RPN is defined as follows [14]:

$$
L\left(\left\{p_{i}\right\},\left\{t_{i}\right\}\right)=\frac{1}{N_{c l s}} \sum_{i} L_{c l s}\left(p_{i}, p_{i}^{*}\right)+\lambda \frac{1}{N_{\text {reg }}} \sum_{i} p_{i}^{*} L_{\text {reg }}\left(t_{i}, t_{i}^{*}\right),
$$

where $L_{c l s}$ and $L_{r e g}$ represent the classification and regression losses respectively, $N_{c l s}$ represents the batch size, $p_{i}$ denotes the predicted objectness score of anchor $i, p_{i}^{*}$ denotes the ground truth label which might be either 1 if the anchor $i$ is labeled as an object or 0 otherwise, $\lambda$ is a weighting factor, $N_{\text {reg }}$ denotes the number of anchor locations, and $t_{i}$ and $t_{i}^{*}$ represent four parameterized coordinates of the predicted and ground truth bounding box respectively. These 
coordinates are given by:

$$
\begin{array}{cc}
t_{x}=\left(x-x_{a}\right) / w_{a}, & t_{y}=\left(y-y_{a}\right) / h_{a}, \\
t_{w}=\log \left(w / w_{a}\right), & t_{h}=\log \left(h / h_{a}\right), \\
t_{x}^{*}=\left(x^{*}-x_{a}\right) / w_{a}, & t_{y}^{*}=\left(y^{*}-y_{a}\right) / h_{a}, \\
t_{w}^{*}=\log \left(w^{*} / w_{a}\right), & t_{h}^{*}=\log \left(h^{*} / h_{a}\right),
\end{array}
$$

where $\left(x_{a}, y_{a}\right),(x, y)$ and $\left(x^{*}, y^{*}\right)$ denote the coordinates of the center of the anchor, predicted bounding box, and ground truth bounding box respectively, and $\left(w_{a}, h_{a}\right),(w, h),\left(w^{*}, h^{*}\right)$ denote the width and height of the anchor, predicted bounding box, and ground truth bounding box respectively.

After extracting the region proposals, some processing steps are applied. Firstly, the proposals are sorted based on their objectness scores. After that, Faster RCNN removes overlapping bounding boxes that may represent the same objects using the Non-Maximum Suppression (NMS) algorithm. NMS checks the sorted list of the region proposals. Then, if the Intersection-over-Union (IoU) ratio between two regions is greater than a pre-specified threshold, NMS drops the region with the lowest objectness score. Following this, the region proposals are applied to the Region-of-Interest (RoI) pooling layer to extract equallysized feature maps corresponding to the extracted proposals (potential objects) before applying them to the final detection network. 
(C) Region of Interest Pooling Layer. The Region-of-Interest (RoI) pooling layer was initially introduced in Fast R-CNN [21]. As shown in Figure 2.16, the ROI pooling layer takes both region proposals and feature map as inputs.
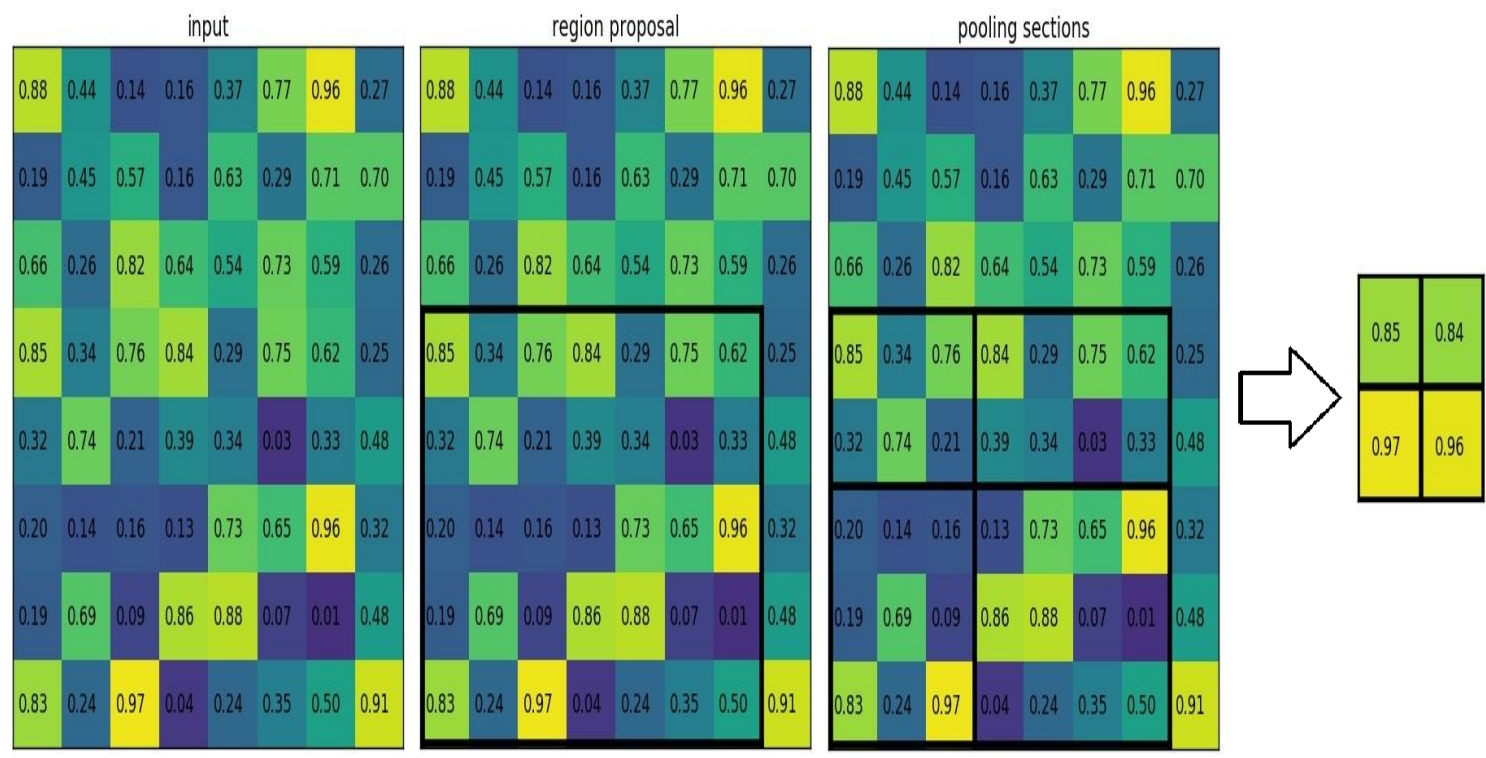

Figure 2.16: Example of a Region-of-Interest pooling operation. The image is reproduced from 33 ]

Then, for each region, the ROI takes the corresponding part of the feature map and divides this part into smaller parts $(e . g . k \times k$, given that the size of the required output is $k \times k$ ). After that, ROI takes the maximum value of each part and outputs a corresponding $k \times k$ feature map. This implementation ensures that whatever the size of the object proposal is, the generated feature map must have the same size $(e . g ., k \times k)$. After extracting the equal-sized feature maps, they can now be applied to the final stage represented by the detection network.

(D) Detection Network. RPN is used in Faster R-CNN to generate region proposals. These proposals represent initial glances of potential objects. However, to determine the classes of these object proposals, Faster R-CNN uses the detection network. The detection network architecture of Faster R-CNN is identical 
to that used in Fast R-CNN. It consists of fully connected layers that branch out into classification and regression networks. The classification network includes a softmax layer, that computes the probability distribution for each proposal over a set of $C+1$ classes including the background class (an uninformative object). The regression network predicts the bounding box coordinates associated with each class and it uses regression to refine the predicted bounding box.

(E) Training Faster R-CNN. Faster R-CNN can be trained using different approaches. For example, we can train RPN and the detection network independently from each other in an alternating training mechanism. Otherwise, both networks can be trained jointly. The latter mechanism is around $25-50 \%$ faster than the alternating training method. The Faster R-CNN approach adopted in our model was trained using the joint end-to-end training mechanism. The loss function used to train Faster R-CNN is the weighted sum of the RPN's loss and the detection network's loss. The former is given in Eq.(2.10). The latter is a combination of two weighted losses. The first loss is similar to the $L_{c l s}$ component of the RPN's loss, but for multi-class classification. The second loss is the regression loss and it is also similar to the $L_{r e g}$ component of the RPN's loss. Each component in the final loss function has a hyper-parameter weighting factor which is used in case we want to give a higher weight to any of the four loss components.

\subsection{Multi-modal Feature Fusion}

After extracting the visual features using Faster R-CNN [14] and the textual answer feature using GRUs, we need a method to combine both features into one joint feature that fully expresses the image and answer relationship. Many approaches have been 
proposed to solve this challenging problem. The most straightforward approaches are the simple element-wise multiplication [34] and concatenation [35] operations. However, although these methods are simple and easy to implement, they usually fail to capture the full correspondence between the elements of the visual and textual modalities. To handle this issue, researchers have proposed the use of the bilinear pooling (or outer product) operation to allow the elements of both features to fully interact with each other and consequently generate a richer joint feature that truly reflects the image and answer relationship. However, applying this method on a complex task like iVQA is still not yet computationally applicable. To explain, if the textual and visual inputs are $m$-dimensional and $n$-dimensional vectors respectively, the generated output of the bilinear pooling operation is $(m \times n)$-dimensional vector. When we apply this vector into a fully connected layer, as shown in Figure 2.17. we need a high dimensional weight matrix $W \in R^{m \times n \times o}$ which is computationally

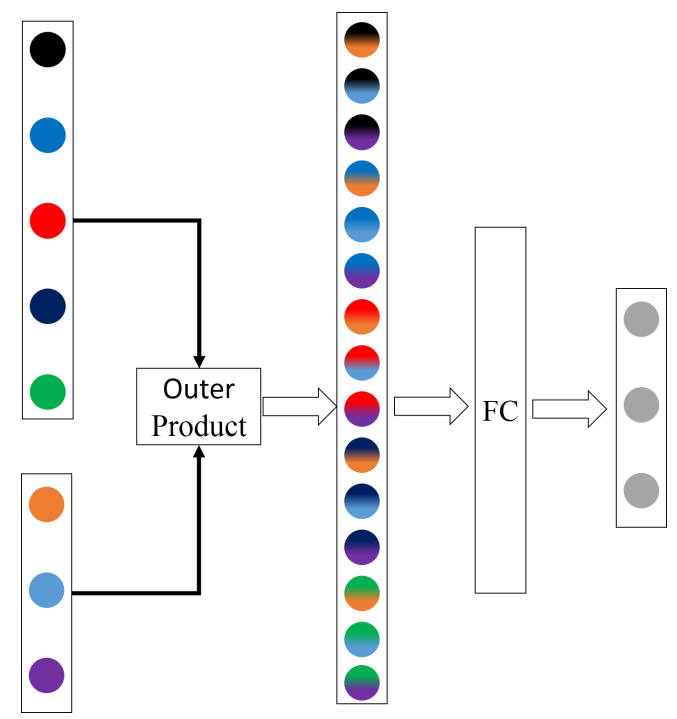

Figure 2.17: The bilienar pooling operation. FC stands for a fully connected layer. The image is reproduced from [36]

impractical as $m$ and $n$ are usually very big. To solve this problem, many algorithms have been proposed. In the following subsections, we present two of the most popular 
bilinear pooling based approaches that are leveraged in our model.

\subsubsection{Multi-modal Low-rank Bilinear Pooling}

Multi-modal Low-rank Bilinear Pooling (MLB) was proposed by Kim et al. [6] to reduce the computational cost of the bilinear pooling operation. MLB first projects the textual feature $x \in R^{m}$ and visual feature $y \in R^{n}$ into the same dimensional space $d$, where $d$ should be not greater than $\min (m, n)$. Following this, MLB fuses the projected feature using the element-wise multiplication as follows:

$$
z=P^{T} f u n c\left(U_{1} x \odot U_{2} y\right),
$$

where $P \in R^{d \times N_{z}}, U_{1} \in R^{d \times m}$ and $U_{2} \in R^{d \times n}$ are learned weights, $\odot$ is the elementwise multiplication, and func denotes an activation function (e.g. Tanh or sigmoid). As shown in Figure 2.18, the MLB decomposes the high dimensional weight matrix,

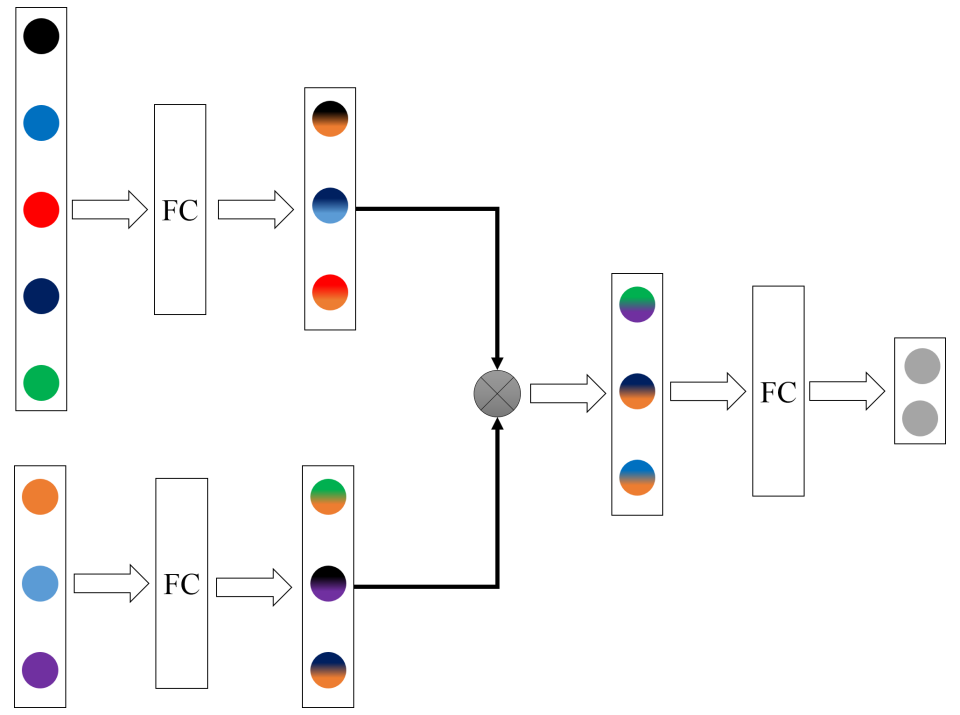

Figure 2.18: The MLB operation. The image is reproduced from [36]

needed for the bilinear pooling operation, into three low-rank projection matrices that can easily fit in any GPU with a good memory. 


\subsubsection{Multi-modal Factorized Bilinear Pooling}

Multi-modal Factorized Bilinear Pooling (MFB) [37] is an improvement to MLB. As shown in Figure 2.19, MFB is divided into two steps; the expand step and the

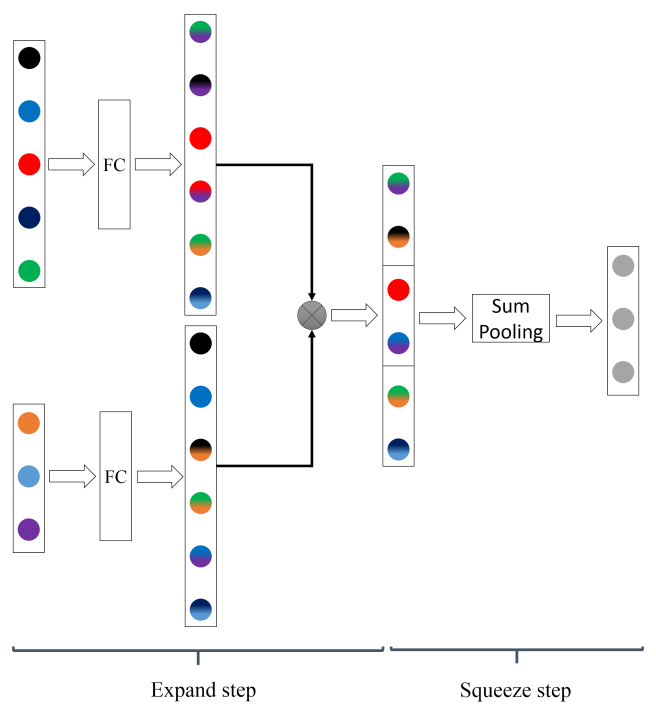

Figure 2.19: Illustration of the MFB operation. The image is reproduced from [36]

squeeze step. In the first step, unlike MLB which constrains the joint dimension $d$ of the projected features to be not greater than $\min (m, n)$, MFB uses two projection matrices; $U_{1}$ and $U_{2}$, to project the visual and textual features into a high-dimensional space. The projected features are then fused into one joint feature as follows:

$$
f=\operatorname{func}\left(U_{1} x \odot U_{2} y\right)
$$

where $U_{1} \in R^{d \times m}$ and $U_{2} \in R^{d \times n}$ are the high dimensional projection matrices. The fused feature is then squeezed using a sum pooling operation with window size $j$ as follows:

$$
z=\operatorname{sum}-\operatorname{pooling}(f, j),
$$


where the sum-pooling function takes the fused feature vector $f$ and divides it into equal non-overlapped parts of size $j$ and then takes the sum of each part to obtain the fused vector $z \in R^{d / j}$. To accelerate the training process and prevent large fluctuations in the values of $z$, MFB normalizes $z$ using a signed square root $z=$ $\operatorname{sign}(z) \sqrt[2]{|z|}$ followed by an $L_{2}$ normalization.

MFB is more computationally expensive than MLB. However, the generated fused feature using MFB is richer than that of MLB and therefore MFB outperforms MLB with VQA [37]. In our iVQA model, we adopt both algorithms at different levels of multimodal feature fusion. 


\section{Chapter 3}

\section{Related Work}

The iVQA task can generally be considered as a combination of image captioning and visual question answering. Therefore, it is essential to study the main techniques developed for both tasks and leverage them for the task of iVQA. In this section, we introduce a brief review of state-of-the-art algorithms used for image captioning and visual question answering (VQA). Then, we present some important methods for visual question generation (VQG), which is very similar to iVQA. We also provide a summary of the main models that adopt attention for sequence learning. Finally, we compare our iVQA models with the current state-of-the-art models.

\subsection{Image Caption Generation}

The standard CNN-RNN [38] model is considered the base for most state-of-the-art image captioning models. It uses a convolutional neural network (CNN) for visual feature extraction and recurrent neural networks (e.g. LSTM) for caption generation. After the success of this simple model, XU et al. [39] improved it by introducing the attention mechanism, which helps the model to selectively focus on important parts of 
an image rather than considering its whole content. This powerful technique inspires researchers to study different attention variants. You et al. presented the semantic attention [40] which helps the model to attend to important semantic concepts corresponding to an input image. After that, Lu et al. [41] proposed a more intuitive attention mechanism, namely adaptive attention. Their model can determine when the visual attention should be enabled if there is a need to consider the visual cue, or disabled when the visual cue does not correspond to the currently generated word. Recently, a considerable improvement in image captioning was achieved by combining both bottom-up and top-down attention [4] into one powerful model. The bottom-up attention model uses Faster R-CNN [14] to extract object-based features. Then, the top-down attention model attends to important objects for each generated word. The models developed for image captioning though, are not directly applicable to other types of tasks such as VQA, VQG, and iVQA. The principles of these techniques, however, can be adapted and generalized.

\subsection{Visual Question Answering}

VQA is similar to iVQA in the way that both tasks should capture complex textual and visual interactions and translate that into written words. Therefore, it is important to leverage state-of-the-art VQA algorithms to boost the performance of iVQA. Unlike image captioning, the primary challenge of VQA is how to extract a multimodal joint feature that sufficiently expresses the textual and visual relationships. To solve this problem, Zhou et al. [35] used the conventional concatenation method to combine textual and visual features. The moderate results of this straightforward method urged researchers to find other advanced techniques. Bilinear-pooling is one of the effective solutions. It allows both visual and textual features to fully interact 
with each other. However, the direct implementation of this method is computationally costly. Consequently, it is not yet applicable to the complex VQA task. Many algorithms were then developed to reduce the number of parameters needed to perform the bilinear-pooling operation. For example, Multi-modal Low-rank Bilinear (MLB) pooling [6] decomposes the high dimensional weight tensor needed for the bilinear-pooling operation into three lower dimensional weight matrices which can easily fit into a Graphic Processing Unit (GPU) memory. Another method to simplify bilinear-pooling is Multi-modal Factorized Bilinear-pooling (MFB) [37]. MFB attempts to capture more complex interactions by first expanding textual and visual features into a high-dimensional feature and then squeezing this feature into a low-dimensional feature. MFB has demonstrated very good performance for many state-of-the-art VQA models. Therefore, we resort to this algorithm to capture the complex interactions between answer and image cues.

Similar to image captioning, the attention mechanism plays an important role to boost VQA performance. Instead of considering the whole content of an image equally, the attention mechanism helps VQA models to focus on parts corresponding to the given question [42]. Many visual attention variants have been proposed for VQA such as the Stacked attention [43] and Hierarchical co-attention [44]. Besides visual attention, semantic attention has also been explored. In this context, Yu et al. [45] designed a multi-level visual-semantic attention model that focuses on both important regions and semantic concepts corresponding to a given question. Nevertheless, as previously discussed, the rich semantic information contained in the questions could make a VQA system produce right answers without any image visual understanding [10], which motivates the new task of iVQA. 


\subsection{Visual Question Generation}

VQG is a new deep learning task that aims to build an artificial intelligence system that can recognize the content of an image and generate relevant questions. The first research work that focused on this task was conducted by Mostafazadeh et al. [7] who repurposed the standard CNN-RNN model for the task of VQG. Similar to that for image captioning, this model became the base for most advanced state-of-the-art VQG models. Correspondingly, Mora et al. [46] also adopted the CNN-RNN model. However, they developed that model to not only generate questions as in [7] but also their corresponding answers. In the same context, Mostafazadeh et al. [47] proposed a model to generate automatic conversation for a given image in the form of questions and their responses. After the great progress achieved by these models, researchers have developed other advanced models by focusing on two main criteria; enhancing question diversity and generating questions targeting important objects in an image. Specifically, Zhang et al. [8] built a more complex model that uses captions generated by DenseCap [48] to generate questions corresponding to main objects in a given image. Li et al. [49] also focused on generating questions associated with salient objects. Their model compares two given images and extracts a distinctive semantic attributes pair that represents the most apparent distinction detected in both images, subsequently, generating a discriminative question that is conditional on both images and the distinguishing attribute pair. Later, Jain et al. [9] further improved question diversity by integrating the vanilla variational autoencoder [50] into their model. Nevertheless, the aforementioned models generate questions merely based on images. Although this is very beneficial for enhancing visual and linguistic understanding, the generated questions may be too generic and lack a full understanding of the given image. Therefore, the iVQA task was proposed in [1]. This task is more challenging 
than VQG because an iVQA model requires a precise understanding of a given image and good reasoning to generate specific questions that are corresponding to an imageanswer pair.

\section{4 iVQA Models}

Two main approaches have been proposed for iVQA [1, 2]. In [1], a powerful model was developed by incorporating two key components; the semantic concepts and dynamic attention. To explain, the iVQA model, as shown in Figure 3.1, can be divided into three stages. In the first stage, the model extracts image features and semantic

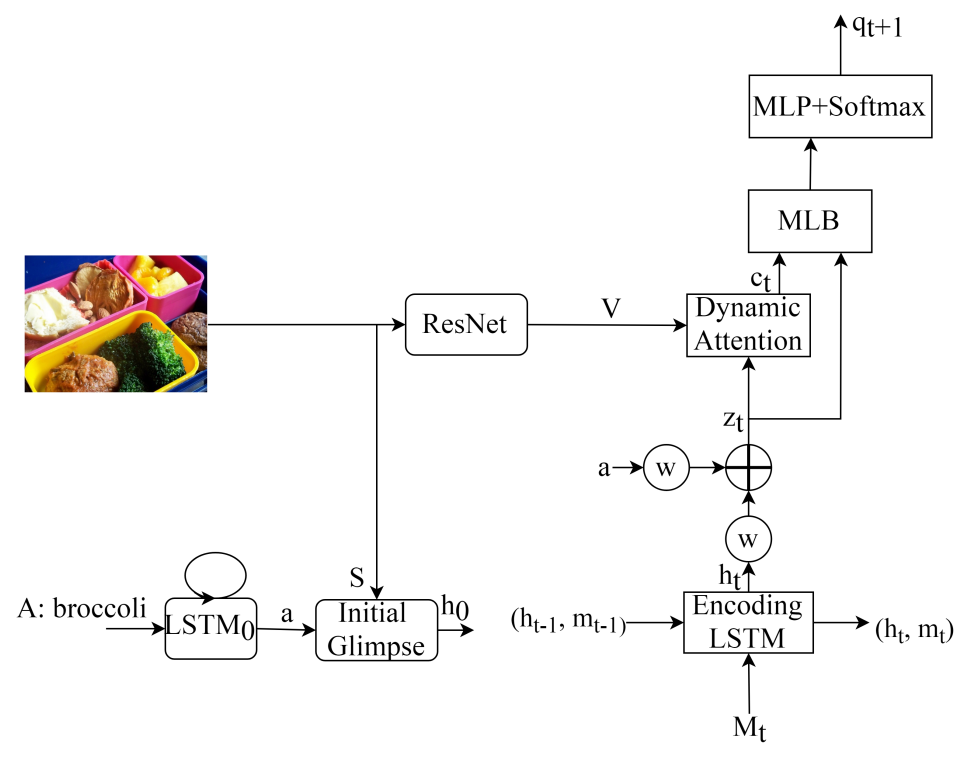

Figure 3.1: Overview of the state-of-the-art iVQA model proposed in [1]. In this figure, $M_{t}$ represents the word embedding of the previously generated question word and $q_{t+1}$ denotes the currently generated question word

concepts. The image features are extracted using the powerfull ResNet-152 model [12]. The semantic concepts are extracted using the 1000 most commonly used words in the captions of the MSCOCO [51] training dataset. For each image, the model predicts the corresponding semantic concepts which are then used in the formulation 
of the generated questions. To extract the answer feature, the model applies the given answer into an LSTM network where the answer feature is obtained by concatenating the hidden and cell state of the last LSTM unit. Subsequently, the model combines the answer feature and semantic concepts into one joint feature which would give the model an initial glance about the nature of the generated question (e.g. what colour or how many). In the second stage, Liu et al. use the dynamic attention mechanism to help the model to dynamically focus on important parts of a given image while generating the question word by word. This is implemented by extracting a textual feature representing both answer and the generated question so far. This textual feature is then combined with the image feature. The output is applied to the attention module (Softmax and sum pooling layers) to generate the attention feature. This feature is further enriched by integrating it with the textual feature (question-answer feature) using MLB [6]. In the third stage, the final feature is decoded using a fully connected layer, followed by a softmax layer which outputs the probability distribution of the currently predicted words. The same procedure is applied until reaching the end of the question.

In the other iVQA model [2], the authors leveraged a variational autoencoder to improve the capability of generating multiple conditional corresponding questions. The model, as shown in Figure 3.2 consists of two parts; an encoder and a decoder. The encoder is composed of an LSTM network that takes a given image and question as inputs and then generates the mean and variance of a multivariate normal distribution. With regards to the decoder, we can see that it is different from [9] where the question generation is mainly based on visual feature and latent representation. This model also leverages the information provided by the answer cue. It first combines the answer feature, visual semantic feature [52], and latent representation to obtain the initial hidden state which is applied to the first LSTM of successive LSTM re- 
peating modules. At each time step, the output of the LSTM is applied into a fully connected layer followed by a Softmax layer to predict the next word which is then encoded and applied to the LSTM, and so on, until generating the whole question. Note that similar to [9], the latent representation can be obtained by applying the reparametrization trick [50].

After developing powerful models for both VQA and VQG, Li et al. [11] exploited the complementary relationship of both tasks and proposed the first model that can work with both VQA and iVQA. This study constituted an important step to confirm the importance of iVQA in improving VQA.

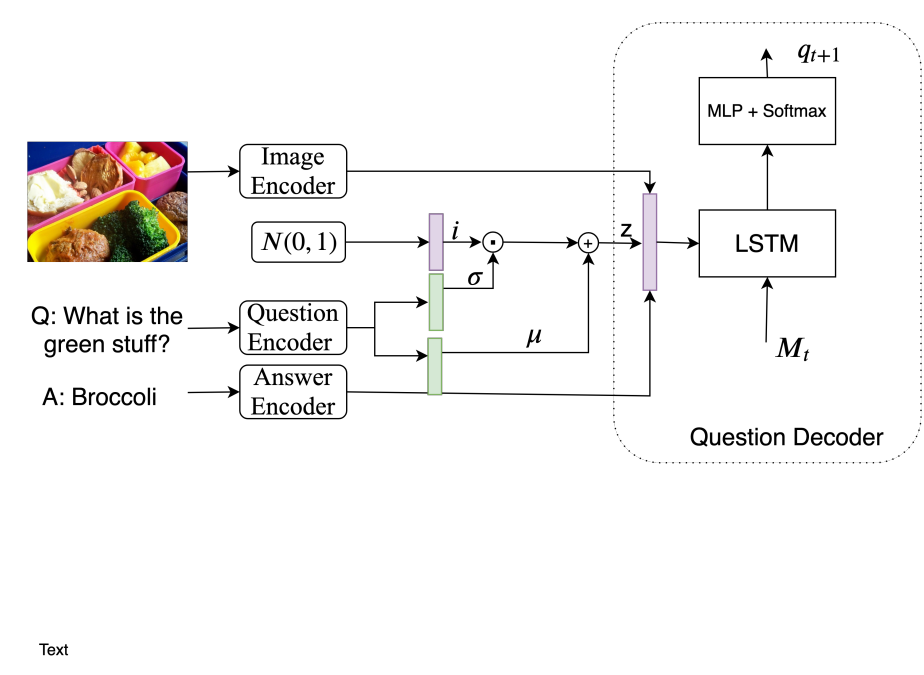

Figure 3.2: Overview of the iVQA model with a variational autoencoder. The figure is reproduced from [2]. In this figure, $z$ is a latent factor, $\mu$ and $\sigma$ represent the mean and variance of $z, M_{t}$ represents the word embedding of the previously generated question word, and $q_{t+1}$ denotes the currently generated question word. 


\subsection{Attention for Sequence Learning}

In terms of using the attention mechanism to replace the traditional recurrent neural network (RNN), Vaswani et al. [53] proposed the Transformer model for machine translation. Transformer was the first attention-based model outperforming the traditional RNN and its variants in the machine translation task. After the great success of Transformer, Devlin et al. [54] developed a bidirectional Transformer based model, namely BERT, and again proved the potential of attention mechanisms for sequence learning by obtaining the state-of-the-art results in several natural language processing tasks. The use of attention mechanism for sequence learning is quite new. To our knowledge, our DMLA model represents the first successful attempt to build an iVQA model that replaces RNNs, used for question modelling, with attention modules for question encoding and decoding.

\subsection{Comparison}

When we look at the previous state-of-the-art iVQA models, we notice that all of them extract the visual representation by using a deep convolutional network model like ResNet [12] or VGG [13]. In contrast, we use Faster R-CNN [14] which has demonstrated great performance in generating visual features for an image at the level of salient objects, as clarified in Figure 3.3. Regarding our SMLA model, we adopt a static attention mechanism that incorporates three attention modules to provide our model with different aspects of semantic and visual knowledge. Interestingly, our SMLA model, with the novel static multi-level mechanism, outperforms the state-ofthe-art iVQA model [1], which is based on dynamic attention, in several benchmarks. Importantly, our attention mechanism only considers the semantic information cor- 
responding to a given answer. This is different from [1, 2], in which the models treat the extracted semantic concepts equally without focusing on those associated with a given answer. To illustrate, if the given answer is "black" and the generated semantic concepts, as shown in Figure 3.3, include "face, feet, arm, black nose, and black eye", then it is more intuitive to assign high importance to the semantic information relevant to "black nose, and black eye" as the predicted question is more likely to be about a black object (e.g. What colour is the bear's nose?).

Besides the aforementioned important points, we emphasize the following differences between our SMLA model and the iVQA model presented in [1]:

- Our semantic representation is richer for two reasons. Firstly, as our visual features are extracted at the level of objects, it is straightforward to align each piece of semantic information with the associated object-based visual feature, while the semantic concepts used in [1] have no direct relation to the visual features of a given image. We can observe this in Figure 3.3 , where each object is directly annotated with a label representing its semantic information. Secondly, we utilize more semantic information (1600 vs 1000 in [1]).

- Unlike the current state-of-the-art iVQA model [1] which uses MLB [6] to fuse visual and question-answer features, we adopt the powerful MFB[1] [37] fusion method to generate more representative fused features of the given image and answer.

- Finally, we better capture long-term dependencies between question words by applying a two-layer GRU model for question generation. In contrast, only a one-layer LSTM model is used in the other iVQA model [1].

\footnotetext{
${ }^{1} \mathrm{~A}$ full description of the MFB and MLB is provided in the Backgournd chapter 2
} 
With regards to the DMLA model, it adopts the dynamic attention mechanism and attends to rich image-answer fused features instead of only visual features as applied in [1]. More importantly, the DMLA model replaces LSTMs, used in [1, 2], with a powerful attention based question encoder and decoder.

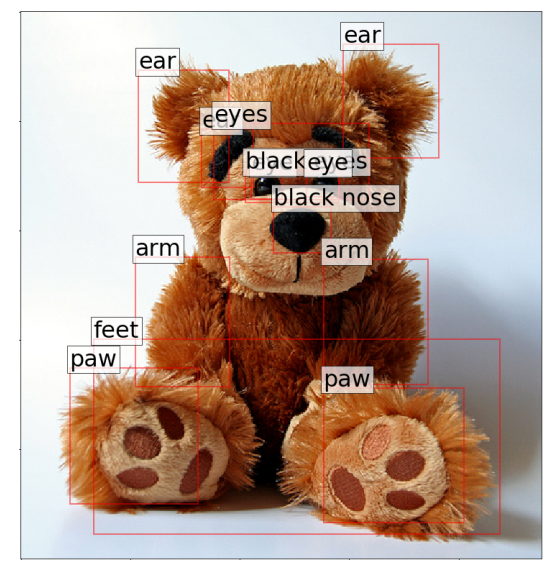

Figure 3.3 


\section{Chapter 4}

\section{The Proposed Models}

In this chapter, we present our novel static and dynamic multi-level attention models for the task of inverse visual question answering. We start with a review of the iVQA task followed by an introduction of the model architectures. Finally, we describe the model training and inference procedures.

\section{1 iVQA task}

Given an image $I$ and an answer phrase $A=\left[w_{1}, \ldots, w_{m}\right]$, the task of inverse question answering (iVQA) generates a corresponding question $Q=\left[q_{1}, \ldots, q_{T}\right]$ by maximizing the following conditional probability:

$$
Q^{\star}=\underset{Q}{\operatorname{argmax}} P(Q \mid I, A ; \theta),
$$

where, $\theta$ denotes the model parameters.

By applying the chain rule, we can rewrite Eq.(4.1) as follows:

$$
Q^{\star}=\underset{Q}{\operatorname{argmax}} \prod_{t} p\left(q_{t} \mid q_{1}, \ldots, q_{t-1}, I, A ; \theta\right) .
$$


The conditional probability $p\left(q_{t} \mid q_{t-1}, \ldots, q_{1}, I, A ; \theta\right)$ can be determined as the hidden output $h_{t}$ of a recurrent neural network (e.g. GRU as used in our model).

\section{2 iVQA Models with Multi-level Attentions}

Our novel models for inverse visual question answering are combinations of multiple attention mechanisms. Comparing to previous iVQA methods, our proposed models have strengths in the following aspects:

1. We encode the input image with a Faster R-CNN module, which generates both object-level semantic labels and the associated regional visual features. For iVQA, this image encoder enables consistent alignment of the visual features and textual answer cue, while providing a suitable foundation for regional selective attention mechanisms.

2. We adopt the powerful Multi-modal Factorized Bilinear-pooling (MFB) 37] method to capture the complex interactions between image and answer features and fuse them into an answer-aware image representation. This representation is further weighted and averaged, using an attention module, to be then applied to the question decoder for next word generation.

3. With regards to our SMLA model, We use two extra auxiliary attention modules to attend to the visual and semantic features based on the given answer cue for partial question encoding.

4. Regarding our DMLA model, we leverage the attention mechanism for sequence learning. To the best of our knowledge, this is the first iVQA model that encodes and decodes questions without the use of RNNs or its variants. 
In the following subsections, we describe the methodology of our SMLA and DMLA models.

\subsubsection{The SMLA Model}

Our SMLA model, as shown in Figure 4.1, is a combination of three static attention mechanisms that work together to select salient visual and semantic information from the given image based on the answer cue. Overall, the main steps in our SMLA

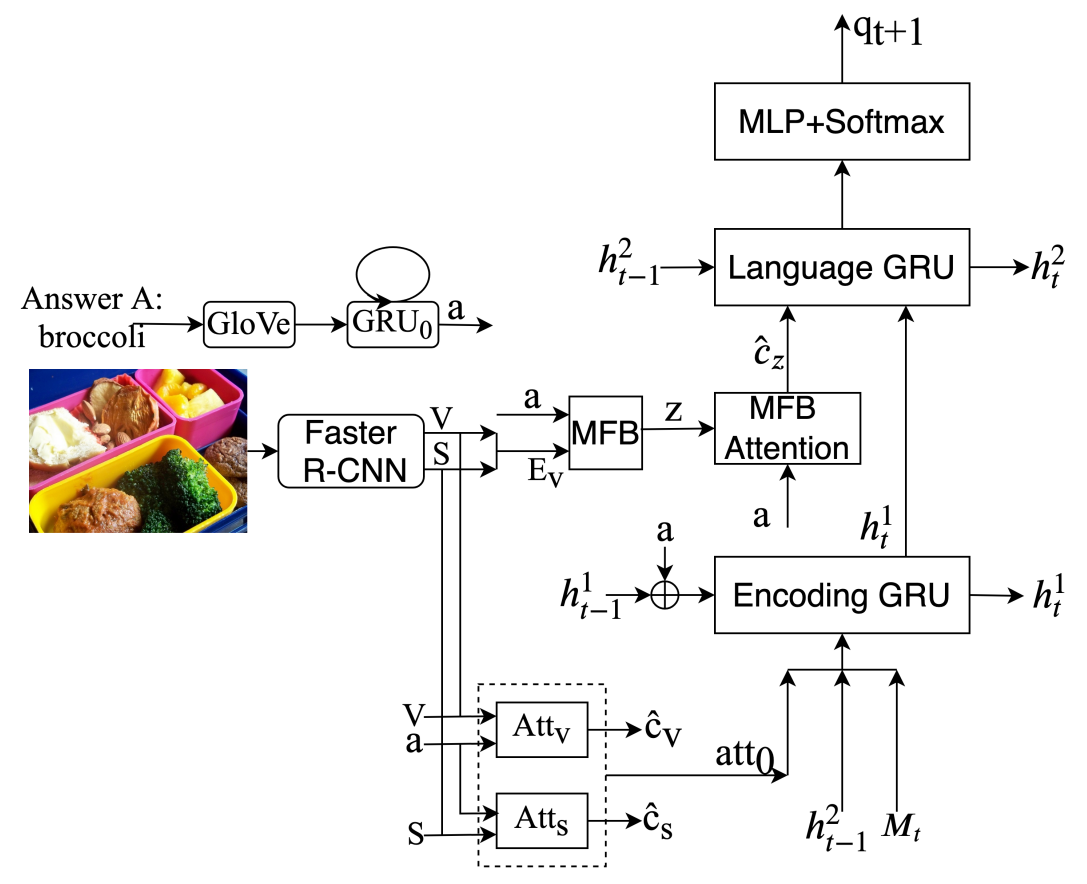

Figure 4.1: The proposed SMLA iVQA model. The model comprises the following components: (1) GRU based answer encoder, which encodes the answer phrase into the semantic embedding space. (2) Faster R-CNN based image encoder, which produces the visual and semantic features of the objects and salient regions. (3) Visual and semantic attention modules, which produces answer attended visual and semantic features. (4) Multi-modal feature fusion (MFB), which fuses the answer cue with the visual and semantic features. (5) MFB attention to attend to the multi-modal fused features (6)Two-layer GRU model, which recurrently generates question words.

approach can be summarized as follows. We first extract the object-based visual 
features with their corresponding semantic information from the given image using a Faster R-CNN based image encoder (bottom-up attention model) [4, 14]. To ensure representation consistency, we use the GloVe encoding to initialize both semantic information and the answers. The answers are further encoded using a GRU, where the answer encoding $a$ is obtained from the last hidden output. Then, we fuse the answer cue with the combined visual and semantic features $E_{v}$ into multi-modal fused features $z$ by using MFB [37. We further attend to this fused image and answer representation $z$ using MFB attention guided by the answer cue. We also extract the important semantic and visual features $\left(c_{s}\right.$ and $\left.c_{v}\right)$ corresponding to the given answer using semantic and visual attention modules $\left(A t t_{s}\right.$ and $\left.A t t_{v}\right)$. Finally, we generate words sequentially by using a two-layer GRU model as follows. (1) The encoder, (Encoding GRU), takes the current word embedding $M_{t}$, the generated question so far $h_{t-1}^{2}$, and the important answer-based visual and semantic features $c_{v}$ and $c_{s}$, respectively and apply this information into a GRU. (2) The attended fused feature $c_{z}$, obtained from the MFB attention module, along with the output of the first GRU $h_{t}^{1}$ are fed to the decoder. (3) The decoder is composed of Language GRU followed by a fully connected network and a softmax layer that generates the probability distribution of the predicted word over the set of vocabulary. Below, we present the key component modules of the proposed SMLA model.

\subsubsection{Semantic Answer Encoder}

Comparing to questions, answers are typically very short phrases such that $A=$ $\left[w_{1}, \ldots, w_{m}\right]$, with $m \leq 3$. Each word in the phrase has semantic meaning. To produce a semantically informative encoding for an answer phrase, we first convert each answer into a list of lowercase tokens. Then, we propose to exploit a natural language processing technique, GloVe [55], to encode each word into a 300-dimensional seman- 
tic embedding vector. Moreover, we use a recurrent neural network, Gated Recurrent Units (GRUs) [29], to capture the dependencies between the sequence of words in an answer, and use the hidden output of the last GRU cell as the answer encoding vector, $a \in R^{H}$.

In fact, answers can be simply encoded by a look-up table as in [11] without the need to include an extra recurrent module (e.g. GRU). As this might not cause substantial problems because of the limited number of answers having more than one word, we found that it is helpful for certain scenarios to use GRUs for extracting answer encodings. To illustrate, if a training look-up table includes both "yellow" and "blue" as answers, an iVQA model should be able to generate corresponding questions whenever either of these answers is given. However, when such a model is given a composed answer like "yellow and blue", we found that the model may not be capable of generating an accurate question compared to a model that uses GRUs to encode answers.

\subsubsection{Semantic and Visual Image Encoder}

Different from previous iVQA approaches [1, 2], which use visual features extracted from deep CNN models (e.g. ResNet [12]), we adopt an object-based regional feature extraction model, Faster R-CNN [14] (with ResNet101 [12] pre-trained on ImageNet [56]), to perform image encoding. Faster R-CNN has been used as a state-of-the-art tool for object detection in computer vision. We use it to extract object-level visual features and associated object labels from input images.

The process of image encoding is illustrated in Figure 4.2. This encoding module has two main steps [4, 14]. In the first step, Faster R-CNN generates region proposals with their corresponding bounding boxes. Specifically, we first pass a given image

\footnotetext{
${ }^{1}$ More details about Faster R-CNN is provided in the Background chapter 2
} 


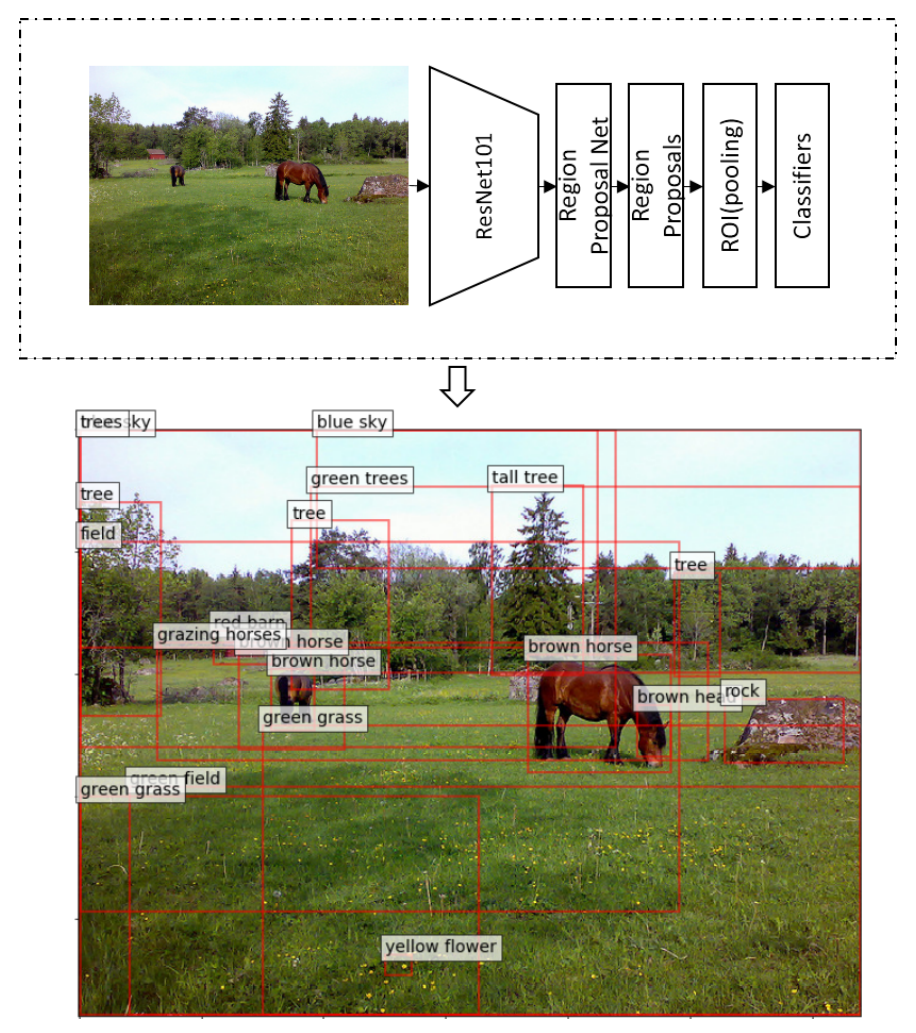

Figure 4.2: Illustration of the Faster R-CNN image encoder module. The detected objects are illustrated in the bottom picture. Each object is localized with a bounding box and annotated with attribute and object labels.

into a residual network (ResNet101) to extract a spatial feature map which is then passed into a Region Proposal Network (RPN) to generate object proposals with a sliding window. For each window, there are multiple anchors that consider different possibilities for a potential object location. At each anchor, RPN uses binary classification to predict the potential of the current anchor forming an object-of-interest. Whenever RPN detects an object, it predicts a bounding box to localize this object. In the second step, the shapes of the region proposals are unified by applying a Regionof-Interest (RoI) pooling layer. The generated visual features are then passed to the final classification module, which predicts objects and their corresponding attribute classes (e.g. brown horse), while refining the corresponding boxes using regression. 
The Faster R-CNN model is trained on the Visual Genome dataset [57]. After training, for each image, we apply the top $k$ most probably predicted object regions into a mean pooling layer to obtain the final visual features, $V=\left\{v_{i} \in R^{2048} \mid i=1, \ldots, k\right\}$. To enhance these visual features [5] with semantic information, we use the predicted object label (e.g. "horse", "tree", etc.) for each of these regions. Similar to the answer encoder, we encode each label into a 300-dimensional embedding vector using GloVe [55]. This leads to $k$ regional object label embeddings, $S=\left\{s_{i} \in R^{300} \mid i=1, \ldots, k\right\}$, representing the textual semantic features of the image in the same semantic space as the answer cue. The visual and semantic features can also be concatenated to form an enhanced image representation $E_{v}$, such that $E_{v}=\left\{E_{v i}=\left[v_{i} ; s_{i}\right] \mid i=1, \ldots, k\right\}$. Different from the image captioning model used in [4] which only uses the visual features $V$ to represent an image, we found that incorporating the textual semantic features with the visual features is beneficial for the iVQA task as it helps the model to reduce the semantic gap between the given image and answer cue.

\subsubsection{Visual and Semantic Attentions}

Given the outputs of the answer encoder and image encoder, $\{a, V, S\}$, question generation is conducted using a two-layer GRU model [4]. In order to provide the GRU model with an initial glimpse of the relevant semantic and visual concepts based on the answer cue and guide the question generation process, we use a dual guiding attention mechanism to attend to the visual and semantic representations in parallel. Focusing on such answer related information could be valuable, especially for complicated questions that involve many salient objects (e.g. , What is the man wearing on his head?). For these kinds of questions, we need a model that can dynamically change its attention at each time step to capture the important objects required for the question generation. However, for iVQA, we argue that this could 
be compensated by having a strong attention mechanism that can determine most of the required salient objects even before the question generation process is started. For this reason, we incorporate the visual and semantic attentions with the MFB attention mechanism to provide our SMLA model with the required answer-aware visual and semantic information at different levels of knowledge.

Visual Attention $\left(A t t_{V}\right)$. The visual attention aims to attend to the most important objects in the image based on the answer cue. To compute the visual attention weights, we fuse the visual and answer features into one joint feature. This can be achieved by using the Multi-modal Low-rank Bilinear (MLB) pooling [6] for each regional visual vector, $v_{i} \in V$, as follows:

$$
\alpha_{i, v}=W_{a t t, v}^{T}\left(\tanh \left(U_{v} v_{i}+b_{v}\right) \odot \tanh \left(U_{a} a+b_{a}\right)\right),
$$

where $W_{a t t, v} \in R^{N}, U_{v} \in R^{N \times N_{v}}$, and $U_{a} \in R^{N \times H}$ are learned weights, $\mathrm{N}$ is the dimension of the joint MLB feature, $b_{v}$ and $b_{a}$ are biases, and $\odot$ denotes the Hadamard product operator. We subsequently pass the visual attention weights to a softmax layer to compute the attended visual representation of the image, $\hat{c_{v}}$ as follows:

$$
\begin{gathered}
\beta_{v}=\operatorname{Softmax}\left(\alpha_{v}\right) \\
\hat{c_{v}}=\sum_{i=1}^{i=k} \beta_{i, v} v_{i}
\end{gathered}
$$

Semantic Attention $\left(A t t_{s}\right)$. We compute the attended semantic representation of the given image in a similar way. The attention weight for each semantic feature 
vector, $s_{i} \in S$, is computed as follows:

$$
\alpha_{i, s}=W_{a t t, s}^{T}\left(\tanh \left(U_{s} s_{i}+b_{s}\right) \odot \tanh \left(U_{a}^{\prime} a+b_{a}^{\prime}\right)\right),
$$

where $W_{a t t, s} \in R^{N}, U_{s} \in R^{N \times 600}$ and $U_{a}^{\prime} \in R^{N \times H}$ are learned weights, $b_{s}$ and $b_{a}^{\prime}$ are biases.

The attended semantic features, $\hat{c_{s}}$, are then computed as follows:

$$
\begin{gathered}
\beta_{s}=\operatorname{Softmax}\left(\alpha_{s}\right) \\
\hat{c_{s}}=\sum_{i=1}^{i=k} \beta_{i, s} s_{i}
\end{gathered}
$$

Both the attended semantic and visual attention features are then concatenated to form the initial attention features, $\operatorname{att}_{0}=\left[\hat{c_{v}} ; \hat{c_{s}}\right]$, as an input to the partial question encoding GRU.

\subsubsection{Multi-Modal Feature Fusion}

To capture complex interactions between the image and answer cue, we use a multimodal factorized bilinear pooling (MFB) operation to fuse the image and answer representation vectors. $\mathrm{MFB}^{2}$ is developed to reduce the computational cost of the standard bilinear-pooling operation between question and visual features for visual question answering. We adopt MFB to fuse the answer encoding vector $a$ and the enhanced image representation $E_{v}$.

MFB is a two-step algorithm with the expansion and squeeze steps. For each enhanced visual feature vector $E_{v i}$ and the answer encoding vector $a$, we first project them into a

\footnotetext{
${ }^{2}$ For more information about MFB, we refer the reader to the Background chapter 2
} 
high dimensional space to fuse and then squeeze the fused vector using a sum pooling operation with window size $j$ as follows:

$$
\begin{gathered}
f_{i}=\left(U_{1} E_{v i}+b_{1}\right) \odot\left(U_{2} a+b_{2}\right) \\
z_{i}=\operatorname{sum}-\operatorname{pooling}\left(f_{i}, j\right),
\end{gathered}
$$

where $U_{1} \in R^{N_{f} \times N_{e}}$ and $U_{2} \in R^{N_{f} \times H}$ are the high dimensional projection weight matrices, $b_{1}$ and $b_{2}$ are the bias parameter vectors, and $j$ is the MFB parameter (e.g., 5).

The MFB feature vector $z_{i}$ is then processed by a signed square root normalization and $L_{2}$ normalization.

\subsubsection{MFB Attention}

Our static multi-level attention mechanism is based on three different attention modules. The visual and semantic attentions are used to consistently promote the salient visual and semantic information associated with an answer. Besides these, we also adopt the MFB attention module $A t t_{m f b}$. Unlike the conventional visual attention in which the model attends to the image, the MFB attention is used to select the relevant parts of the multi-modal fused features $z$. This important feature, obtained from the MFB module, is a combination of the visual, semantic, and answer information. We think it is important to utilize this rich feature in our model. However, similar to visual features $V$, not all the information included in $z$ is relevant. Consequently, we avoid feeding our model with unnecessary information by using the MFB attention module to attend to the important parts of the fused features $z$ based on a given answer. 
The MFB attention weights are determined by two factors; the answer vector $a$ and the fused feature vectors $\left\{z_{i}\right\}$. The fused feature vectors fuse the answer cue into each regional image representation vector with the MFB operation and it is used to extract the crucial image and answer interaction. Mathematically, we compute the MFB attention weights as follows:

$$
\alpha_{i, z}=W_{a t t}^{T}\left(\tanh \left(W_{z} z_{i}+b_{z}\right) \odot \tanh \left(U_{a}^{\prime \prime} a+b_{a}^{\prime \prime}\right)\right),
$$

where $W_{a t t} \in R^{N}, W_{z} \in R^{N \times N_{z}}, U_{a}^{\prime \prime} \in R^{N \times H}, b_{z}$, and $b_{a}^{\prime \prime}$ are the model parameters. The attention weights are computed for each of the image-answer fused feature, $z_{i} \in z$, and further normalized by a softmax function:

$$
\beta z=\operatorname{softmax}\left(\alpha_{z}\right) \text {. }
$$

Then, different from other iVQA approaches [1, 2] where the models attend only to the image $V$, we attend to the image-answer fused features $z$. The attended fused feature $\hat{c_{z}} \in R^{N_{z}}$ is then computed by taking the weighted sum of the image-answer fused features $z$ as follows:

$$
\hat{c_{z}}=\sum_{i=1}^{i=k} \beta_{i, z} z_{i}
$$

Having computed the attended visual, semantic, and image-answer fused features, the SMLA model should have a reasonable understanding of the given image and its relationship with the answer cue. Consequently, it is ready for the recurrent question generation process as explained in the next subsection. 


\subsubsection{Recurrent Question Generation}

Given the concatenation of the attended visual and semantic vectors, att 0 , and the attended image-answer fused vector $\hat{c_{z}}$, the model recurrently generates the sequence of question words, $\left[q_{1}, \ldots, q_{T}\right]$, using a two-layer Gated Recurrent Unit (GRU). The first GRU layer works as a question encoder while the second one acts as a question decoder. We describe the question encoding and decoding processes below.

Question Encoder (Encoding GRU). At each recurrent step, a GRU encoder is deployed to encode the current partial question generated, aiming to capture the longterm dependencies in the question word sequence for next word generation. Given the question word $q_{t}$ generated from the immediate previous recurrent step, we first embed $q_{t}$ into the same semantic embedding space as the answer cue with a pretrained GloVe model [55], which produces an embedding vector $M_{t} \in R^{300}$. Specifically, we perform the word embedding as follows:

$$
M_{t}=W_{e m b} 1_{q_{t}}+b_{e m b}
$$

where $W_{e m b} \in R^{300 \times N_{w}}$ is initialized from the word embedding matrix produced by GloVe, $N_{w}$ denotes the vocabulary size, $b_{e m b}$ is the embedding bias vector, and $1_{q t}$ denotes an one hot vector with length $N_{w}$, which has a single 1 for the entry corresponding to word $q_{t}$.

Then, we update the recurrent question encoding state $h_{t}^{1}$ by using the concatenation of the word embedding $M_{t}$, answer attended visual and semantic features $a t t_{0}$, and the previous question decoding state $h_{t-1}^{2}$ as input:

$$
r_{t}=\left[M_{t}, h_{t-1}^{2}, a t t_{0}\right], \quad h_{t}^{1}=G R U_{1}\left(r_{t}, h_{t-1}^{1}+a\right),
$$


where $r_{t}$ is a context vector that provides the model with the necessary information involved in the previously generated question words and importantly, the answer related visual and semantic features represented by $a t t_{0}$. The hidden state $h_{t-1}^{1}$ is set to $a$ at $t=0$. Note, we further emphasize the answer cue by adding it to the previous hidden state vector of the encoder $G R U_{1}$.

Question Decoder (Language GRU). In the top GRU decoding layer, we concatenate the attended image-answer fused feature vector $\hat{c_{z}}$ with the output state of the GRU encoder $h_{t}^{1}$ to update the state of the recurrent GRU decoder as follows:

$$
h_{t}^{2}=G R U_{2}\left(\left[\hat{c_{z}} ; h_{t}^{1}\right], h_{t-1}^{2}\right)
$$

where $h_{t-1}^{2}$ is the previous question decoding state at step $t-1$ and it is set to the answer encoding vector $a$ at $t=0$.

To predict the next word probability distribution over the set of vocabulary words, we further pass the state vector of the GRU decoder through a fully connected layer followed by a softmax layer:

$$
\hat{p}=\operatorname{softmax}\left(W_{d e c} h_{t}^{2}+b_{d e c}\right)
$$

where $W_{d e c} \in R^{N_{w} \times N_{h}}$ and $b_{\text {dec }} \in R^{N_{w}}$ are the model parameters. With a beam size $=1$, the next $(t+1)$-th word can then be generated as:

$$
q_{t+1}=\operatorname{VOC}(\hat{j}), \quad \hat{j}=\operatorname{argmax}_{j} \hat{p}_{j}
$$

where VOC denotes the vocabulary table. To generate a complete question, we repeat the whole encoding-decoding process for T steps. 


\subsubsection{The DMLA Model}

Our DMLA model is proposed as another potential solution for the task of iVQA. The importance of this model can be especially observed while generating complicated questions where the DMLA model can dynamically focus on the necessary visual and textual information for the current question generation state. The DMLA model, as shown in Figure 4.3, deploys sequence attention modules for question encoding and decoding instead of using the conventional sequence learning methods such as RNNs, or its variants (e.g. , GRUs).

Similar to the SMLA model, the DMLA model encodes the input image using Faster R-CNN [4]. However, the DMLA model does not use the semantic information for visual feature enhancement. Moreover, the answer encoder used in the DMLA model is similar to that used in the SMLA model. The difference is limited to the size of the GRU units. Furthermore, we adopt the same MFB module used in our SMLA model. However, we do not utilize any semantic information generated from the object labels as we did in the SMLA model.

Although there are some similarities between the SMLA and DMLA models, there are many important differences. Specifically, our DMLA model does not use the visual and semantic attentions. Alternatively, it deploys dynamic attention to a novel GRUfree module for question generation as explained below.

\subsubsection{GRU-free Recurrent Question Generation}

The question generation approach used in the DMLA model is GRU-free and is com-

posed of three main components: sequence attention for question encoding, dynamic attention, and sequence attention for question decoding. 


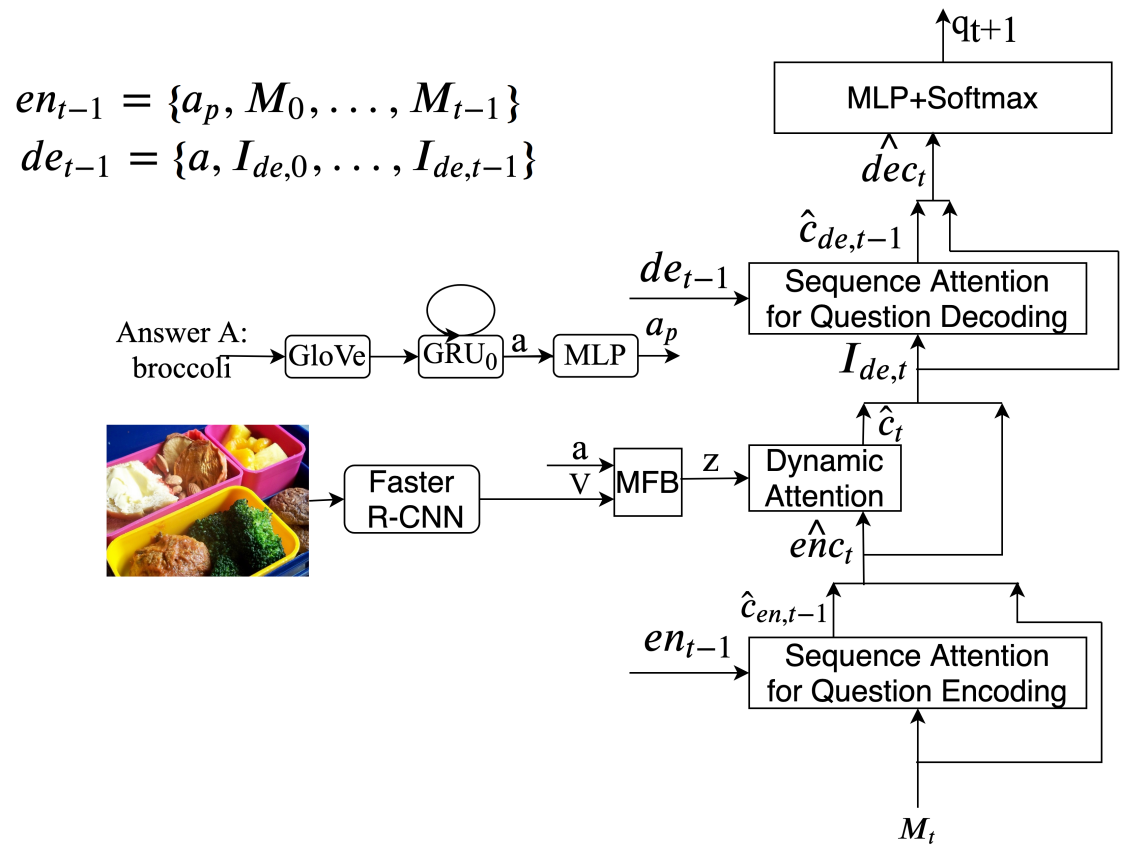

Figure 4.3: The proposed DMLA iVQA model. The model comprises the following components: (1) GRU based answer encoder, which encodes the answer phrase into the semantic embedding space. (2) Faster R-CNN based image encoder, which produces the visual features of the object and salient regions. (3) Multi-modal feature fusion (MFB), which fuses the answer cue with the visual features. (4) Two layers of sequence attentions connected by a dynamic attention mechanism to recurrently generate the question words.

Sequence Attention for Question Encoding. At each time step, an attention module is deployed to generate a context vector $\hat{c}_{e n, t-1}$ representing the attended partial question-answer encoding. The main advantage of this mechanism over the conventional GRUs is that, at each recurrent step, the model can decide not only the importance of the previously generated question words but also the relevance of the answer cue to the current generation state. Before computing the sequence attention weights at $t=0$, we further encode the answer $a$ to have the same dimensional space 
as the question word $q_{t}$ using a fully connected layer as follows:

$$
a_{p}=\tanh \left(W_{a_{p}} a+b_{a_{p}}\right),
$$

where $W_{a_{p}} \in R^{300 \times H}$ is a learned weight matrix and $b_{a_{p}}$ is the corresponding bias vector. At time step $t$, we embed $q_{t}$, generated in the immediately previous recurrent step $t-1$, into a 300-dimensional vector $M_{t}$ by applying Eq.4.14. After that, we take the encoded answer $a_{p}$ and the generated partial question $\left\{M_{0}, \ldots, M_{t-1}\right\}$ to produce the set $e n_{t-1}=\left\{a_{p}, M_{0}, \ldots, M_{t-1}\right\}$. Subsequently, we compute the attention weight for each vector $e n_{i} \in e n_{t-1}$ as follows:

$$
\alpha_{i, t-1}=W_{a t t, e n}^{T}\left(\tanh \left(U_{e n} e n_{i}+b_{e n}\right) \odot \tanh \left(U_{M} M_{t}+b_{M}\right)\right),
$$

where $W_{\text {att }, \text { en }} \in R^{N_{s}}, U_{\text {en }} \in R^{N_{s} \times 300}, U_{M} \in R^{N_{s} \times 300}, b_{\text {en }}$, and $b_{M}$ are the model parameters. The attended partial question-answer encoding $\hat{c}_{e n, t-1}$, is then computed as follows:

$$
\hat{c}_{e n, t-1}=\sum_{i=0}^{i=t} \beta_{i, t-1} e n_{i}, \quad \beta_{t-1}=\operatorname{softmax}\left(\alpha_{t-1}\right),
$$

where $i$ indexes the set of $e n_{t-1}\left(e . g . e n_{0}=a_{p}\right.$ and $\left.e n_{1}=M_{0}\right)$. Finally, we concatenate the current word embedding $M_{t}$ and the attended partial question-answer encoding $\hat{c}_{e n, t}$ to form the question-answer context vector $e \hat{n} c_{t} \in R^{600}$ as follows:

$$
e \hat{n} c_{t}=\left[\hat{c}_{e n, t-1}, M_{t}\right]
$$

Following this, we apply the vector $e \hat{n} c_{t}$ to the dynamic attention module as explained in the next subsection. 
Dynamic Attention. Given the current question-answer context vector, we use a soft attention module to dynamically attend to the image-answer fused features 23. The dynamic attention module used in the DMLA model is similar to the MFB attention module used in the SMLA model in the context that both attend to the rich image-answer fused features $z$. However, while the MFB attention is statically determined at the first time step based on the answer cue, the dynamic attention has the ability to change its focus based not only on the answer cue, but also the current question generation state, $e \hat{n} c_{t}$. Mathematically, the dynamic attention weight for each $z_{i} \in z$, at time step $t$, is computed as follows:

$$
\alpha_{i, t}^{\prime}=W_{a t t}^{T}\left(\tanh \left(W_{z} z_{i}+b_{z}\right) \odot \tanh \left(W_{e} e \hat{n} c_{t}+b_{e}\right)\right)
$$

where $z_{i}$ represents an image-answer fused vector, and $W_{a t t} \in R^{N}, W_{z} \in R^{N \times N_{z}}$, $W_{e} \in R^{N \times 600}, b_{z}$, and $b_{e}$ are the model parameters.

Unlike the other iVQA approaches [1, 2] in which the models immediately attend to the image representation $V$, we compute the dynamically attended image-answer context vector $\hat{c}_{t} \in R^{N_{f}}$ by taking the weighted sum of the enriched image-answer fused features $z$ as follows:

$$
\hat{c}_{t}=\sum_{i=1}^{i=k} \beta_{i, t}^{\prime} z_{i}, \quad \beta_{t}^{\prime}=\operatorname{softmax}\left(\alpha_{t}^{\prime}\right)
$$

Note that the attention weights are determined by two factors $\left\{z_{i}\right\}$ and $e \hat{n} c$. The former $\left\{z_{i}\right\}$ fuse the answer cue into each regional image representation vector with the MFB operation and it is used to extract the complex image and answer interactions, while the latter dynamically captures the current question-answer context as

\footnotetext{
${ }^{3}$ To compute $z_{i} \in z$, we apply the same equations described in the subsection 4.2.1.4. However, in Eq. 4.9 , we replace the enhanced visual features $E_{v}$ by the image representation $V$
} 
it changes over time. Hence, the role of the dynamic attention module is to utilize this information to focus only on salient image-answer fused features corresponding to the context of visual objects, answer, and generated question so far.

Sequence Attention for Question Decoding. Given the current question-answer and the attended image-answer contexts, $\left\{e \hat{n} c_{t}, \hat{c}_{t}\right\}$, we deploy a sequence attention module for question decoding. This module is proposed as a strong alternative to the Language GRU used in the SMLA model. The output of this sequence attention is comparable to the question decoding state $h_{t-1}^{2}$, which is used in the SMLA model to equip the question decoder with the context of visual and textual information generated from the previous recurrent steps. However, the output of the sequence attention for question decoding is more selective as it only considers the corresponding visual and textual information needed for the current question decoding step. To compute the attention weights, we first concatenate the dynamically attended image-answer context $\hat{c}_{t}$ with the question-answer context vector $e \hat{n} c_{t}$ to obtain the input decoding vector $I_{d e, t}$. Next, we take the answer $a$ with the previously generated input decoding vectors, to produce the set $d e_{t-1}=\left\{a, I_{d e, 0}, \ldots, I_{d e, t-1}\right\}$, for question decoding. After that, the attention weight for each vector, $d e_{i} \in d e_{t-1}$, is computed as follows:

$$
\alpha_{i, t-1}^{\prime \prime}=W_{a t t, d e}^{T}\left(\tanh \left(U_{d e} d e_{i}+b_{d e}\right) \odot \tanh \left(U_{I_{d e}} I_{d e, t}+b_{I_{d e}}\right)\right)
$$

where $W_{a t t, d e} \in R^{N_{s}}, U_{d e} \in R^{N_{s} \times H}, U_{I_{d e}} \in R^{N_{s} \times H}, b_{d e}$, and $b_{I_{d e}}$ are the model parameters. The attended partial question decoding $\hat{c}_{d e, t-1}$, is then computed from the following equation:

$$
\hat{c}_{d e, t-1}=\sum_{i=0}^{i=t} \beta_{i, t-1}^{\prime \prime} d e_{i}, \quad \beta_{t-1}^{\prime \prime}=\operatorname{softmax}\left(\alpha_{t-1}^{\prime \prime}\right),
$$


where $i$ indexes the set $d e_{t-1}\left(e . g\right.$. $d e_{0}=a$ and $\left.d e_{1}=I_{d e, 0}\right)$.

Following this, we concatenate the attended partial question decoding, $\hat{c}_{d e, t-1}$, with the vector $I_{d e, t}$ to get the partial question decoding vector $\hat{d e} c_{t}$. Similar to the last decoding stage used in the question decoder of our SMLA model, we feed the vector $\hat{d e} c_{t}$ into a fully connected network followed by a softmax layer to predict the next word probability distribution over the set of vocabulary words as follows:

$$
\hat{p}=\operatorname{softmax}\left(W_{d e c} \hat{d e c} c_{t}+b_{d e c}\right),
$$

where $W_{d e c} \in R^{N_{w} \times 2 H}$ and $b_{d e c} \in R^{N_{w}}$ are model parameters. With a beam size $=1$, we select the word with the highest probability to be injected to the next generation step.

In the next two subsections, we elaborate the training and inference procedures used in our SMLA and DMLA models.

\subsection{Training}

With the exception of Faster R-CNN, We perform an end-to-end deep training over a set of training instances. The $i$-th instance is a triplet that includes an image $I^{i}$, an answer phrase $A^{i}=\left[w_{1}^{i}, \ldots, w_{m_{i}}^{i}\right]$, and a ground truth question $Q^{i}=\left[q_{1}^{i}, \ldots, q_{T_{i}}^{i}\right]$. We train the proposed model by minimizing the cross-entropy loss as follows:

$$
L(\theta)=-\sum_{i} \frac{1}{T_{i}} \sum_{t=1}^{t=T_{i}} \log P\left(q_{t}^{i} \mid q_{1}^{i}, \ldots, q_{t-1}^{i} ; I^{i}, A^{i}\right)
$$

where $\theta$ represents all the model parameters, and $P\left(q_{t}^{i} \mid q_{1}^{i}, \ldots, q_{t-1}^{i} ; I^{i}, A^{i}\right)$ denotes the conditional probability of the word $q_{t}$ for the question $i$, given the corresponding image, answer and the generated question so far and can be determined from Eq.4.17) 
and Eq.4.27) for the SMLA and DMLA models, respectively. Note that, with the exception of the encoding GRU and Language GRU, we use $l_{2}$-regularization for all the fully connected networks used in our SMLA model. Consequently, another $l_{2^{-}}$

regularization loss should be added to the cross-entropy function defined in Eq.(4.28).

\subsection{Inference}

During the test phase, given the trained model and an image-answer pair, we recurrently compute the probability distribution of the next word in the question sequence over our vocabulary. We use a beam size of 1 (greedy search) to select the most probable word as the output, as in Eq.44.17) and Eq.4.27) for the SMLA and DMLA models, respectively. Generally, the question generation terminates after generating the question mark "?". However, for a very long question, we stop the generation process after $T$ steps. During decoding, we further post-process the generated questions by discarding duplicated words that appear in two or more consecutive positions. 


\section{Chapter 5}

\section{Evaluation Metrics}

We evaluate our models using the standard evaluation metrics [51], specifically, BLEU, METEOR, ROUGE-L and CIDEr. These metrics are used extensively to evaluate image caption models [51]. They can also be leveraged for any sequence prediction task like iVQA [1]. In this section, we present detailed information about each of the standard evaluation metrics.

\subsection{BLEU}

BLEU [58] (BiLingual Evaluation Understudy) was originally developed to provide an automatic tool for machine translation evaluation. BLEU determines the quality of machine-generated sentences by comparing them with given references (e.g. sentences generated by humans). Then, the higher BLEU score indicates higher similarities between the generated sentences and the references and consequently, a good performing system.

In order to compute the BLEU score, we need to determine two main values, the modified n-gram precision and brevity penalty. The modified n-gram precision value 
between candidate sentences and corresponding reference sentences of a corpus is given as follows:

$P_{n}=\frac{\sum_{C \in \text { Candidates }} \sum_{n-\text { gram } \in C} \min (\operatorname{Count}(n-\operatorname{gram}), \text { Max_Ref_Count }(n-\text { gram }))}{\sum_{C \in \text { Candidates }} \sum_{n-\text { gram } \in C} \operatorname{Count}(n-\text { gram })}$,

where n-gram denotes the set of possible ordered words $w_{k}$ (each of size $\mathrm{n}$ ) that can be extracted from a sentence, Count (n-gram) represents the number of an ngram that exists in the generated candidate sentence (C), Max_Ref_Count (n-gram) is determined by computing the maximum number of the n-gram that appear in each of the given references. The same procedure used to compute BLEU scores for machine translation can be generalized for other sequence prediction tasks like iVQA. Specifically, for each image, we take the generated candidate question $c_{i}$ and the corresponding references $r e f_{i j}$ and substitute these values in Eq.(5.1) as follows [51]:

$$
P_{n}=\frac{\sum_{i} \sum_{k} \min \left(h_{k}\left(c_{i}\right), \max _{j \in m} h_{k}\left(r e f_{i j}\right)\right)}{\sum_{i} \sum_{k \in \Omega} h_{k}\left(c_{i}\right)}
$$

where $\Omega$ denotes the set of n-grams of size $n$ extracted from a given sentence. $h_{k}\left(c_{i}\right)$ is the count of an n-gram $w_{k}$ appearing in the candidate $c_{i}, h_{k}\left(r e f_{i j}\right)$ is the count of $w_{k}$ appearing in the corresponding reference $r e f_{i j}$, and $m$ denotes the number of references given for a specific candidate.

As an example, we consider the following two references:

Reference 1: is the cat sleeping

Reference 2: is the dog eating

and the generated candidate question :

Candidate: is there a cat

We use Eq. (5.2) to compute the modified uni-gram precision of this example, we 
compare the candidate with the first and second reference. We notice that only the words "is" and "cat" appear in the candidate and references. Now, we determine the maximum number of times the word "is" exists in each reference. Subsequently, we compare the result with the number of times the word "is" occurs in the candidate and then take the minimum value (1 in this case). We apply the same procedure to determine the co-occurrence of the word "cat" in both candidate and references and get a result of 1 . After that, we add up both results $(1+1)$ and divide that by the total number of uni-grams in the candidate question (4). The modified uni-gram precision is then 0.5 .

The bi-gram, 3-gram, and 4-gram precision for our example are zeros as there is no co-occurrence of any n-gram match between the candidate and the given references when $n>1$. However, assume we have another reference in our example like Reference 3: is there a cat on the bed. Then, the n-gram precision is 1 whatever the value of $n$ is. This is an inaccurate result because we have obtained a perfect value for the n-gram precision although there is a difference between the candidate and reference 3. To remedy this issue, we apply a brevity penalty to prevent assigning a perfect n-gram precision value for such short candidate questions. The brevity penalty BP is defined as follows:

$$
B P=\left\{\begin{array}{ll}
1 & \text { if } l_{C}>l_{R E F} \\
e^{1-\frac{l_{R E F}}{l_{C}}} & \text { if } l_{C} \leq l_{R E F}
\end{array},\right.
$$

where $l_{C}$ represents the length of all generated candidates, and $l_{R E F}$ is the length of all considered references. It should be noted that the reference with the closest length to the corresponding candidate is considered among the others for computing $l_{R E F}$. Having calculated the modified n-gram precision $P_{n}$ between all candidates and their 
corresponding references and the brevity penalty $B P$, we can compute the BLEU score by using the following formula:

$$
B L E U_{N}=B P \exp \left(\sum_{n=1}^{N} w_{n} \log P_{n}\right)
$$

where $N$ is a constant (e.g. 4 in our implementation) and $w_{n}=\frac{1}{N}$ is a uniform weight.

BLEU has proved to be an important automatic evaluation metric that is correlated with human assessments. However, it has some drawbacks that may cause an inaccurate evaluation. For example, with high order n-grams (e.g. $\mathrm{n}=4)$, the metric considers every single word in a given n-gram to be equally important [59]. Although this may be reasonable, it is not highly correlated with human judgments. To illustrate, if the generated candidate question "is the cat sleeping" and the given reference is "is this cat sleeping", the corresponding 4-gram precision value is zero as there is no 4gram match between both sentences. Consequently, this correct generated candidate question has the same 4-gram precision score as any other wrong generated candidate. Based on that, although BLEU has become a crucial evaluation tool, it is always beneficial to use it with other evaluation metrics to precisely test the robustness of the developed algorithms.

\subsection{METEOR}

METEOR [60] (Metric for Evaluation of Translation with Explicit ORdering) is another automatic evaluation metric developed initially for the machine translation task. Similar to other metrics, it judges the quality of generated candidate sentences by their similarities to given references. However, there are many improvements achieved 
by METEOR such as considering the linguistic equivalence (e.g. synonym and stemming) which was ignored in other metrics (e.g. BLEU). In addition, METEOR adopts both precision and recall while BLEU only considers precision.

To compute the METEOR score [51, 60], we should first determine the alignment between the candidates and references. To achieve that, we look for maximum unigram matches between a candidate and its corresponding references. The unigram match is not limited to identical words, but it also examines possible synonyms, using WordNet [61], and stemming. Having identified the alignment $(m)$ between a candidate and corresponding references, we can determine the uni-gram recall $\left(R_{m}\right)$ and precision $\left(P_{m}\right)$ as follows:

$$
R_{m}=\frac{|m|}{\sum_{k} h_{k}\left(r e f_{i j}\right)}, \quad P_{m}=\frac{|m|}{\sum_{k} h_{k}\left(c_{i}\right)},
$$

where $h_{k}\left(c_{i}\right)$ and $h_{k}\left(r e f_{i j}\right)$ represent the number of times an unigram $w_{k}$ occurs in the candidate $\left(c_{i}\right)$ and the corresponding reference $\left(r e f_{i j}\right)$ respectively.

Next, we can compute the F-mean score as the harmonic mean of precision and recall as follows:

$$
F_{\text {mean }}=\frac{P_{m} R_{m}}{\alpha P_{m}+(1-\alpha) R_{m}},
$$

where $\alpha$ is a hyper-parameter.

METEOR handles the word ordering differences between the candidate and corresponding reference by imposing a fragmentation penalty that is computed as follows:

$$
\text { Pen }=\gamma\left(\frac{|c h|}{|m|}\right)^{\theta}
$$


where $c h$ denotes the number of identical chunks in both candidate and reference, $\gamma$ and $\theta$ are hyper-parameters. From Eq.5.7, we can notice that when the order of words is identical in both candidate and reference, the fragmentation penalty is very low as in this case we only have one contiguous chunk. In contrast, if the candidate is "word $d_{2}$, word $_{3}$, word $_{4}$, word $_{1}$ " and the given reference is " $\operatorname{word}_{1}$, word $_{2}$, word $_{3}$, word $_{4}$ ", then the number of matching chunks is 4 which is identical to the number of uni-gram matches. Consequently, the fragmentation penalty should be very high, causing a low METEOR score. After computing the $F_{\text {mean }}$ score and fragmentation penalty, the final METEOR score is defined as follows:

$$
M E T E O R=(1-P e n) F_{\text {mean }}
$$

\subsection{ROUGE}

ROUGE [62, 51] (Recall-Oriented Understudy for Gisting Evaluation): represents a set of evaluation metrics that was initially developed for assessing the quality of machine-generated text summarization. The ROUGE metrics can be divided into four kinds, namely $R O U G E_{N}, R O U G E_{L}, R O U G E_{S}$, and $R O U G E_{W}$. In this section, we focus on both $R O U G E_{N}$ and $R O U G E_{L}$.

- $R O U G E_{N}$ : is determined by computing the n-gram recall between the generated candidate and corresponding references as follows:

$$
\operatorname{ROUGE} E_{N}=\frac{\sum_{j} \sum_{k \in \Omega} \min \left(h_{k}\left(c_{i}\right), h_{k}\left(r e f_{i j}\right)\right)}{\left.\sum_{j} \sum_{k \in \Omega} h_{k}\left(r e f_{i j}\right)\right)},
$$

where $\Omega$ denotes the set of the sentence's n-grams, $h_{k}\left(c_{i}\right)$ and $h_{k}\left(r e f_{i j}\right)$ are the number of times an n-gram $w_{k}$ occur in the candidate $c_{i}$ and reference $r e f_{i j}$ 
respectively. Note that $R O U G E_{N}$, to some extent, is similar to BLEU in the context that both depend on n-grams matches. However, BLEU mainly adopts the precision rather than the recall score.

- ROUGE $E_{L}$ : is another variant of ROUGE. In contrast to ROUGE $E_{N}$ and BLEU, which depend on the n-gram matches, $R O U G E_{L}$ is based on the Longest Common Subsequence (LCS). LCS is defined as the longest sequence of words that appear in the same order in both the generated candidate and the corresponding reference. For example, if the candidate is "is the man eating banana" and the reference is "is the woman eating banana", LCS is "is the eating banana". We notice that LCS is more flexible compared to the n-gram for two reasons. Firstly, the length of the subsequence is dynamic while it is restricted to $\mathrm{n}$ words in n-grams. Secondly, the ordered words of an n-gram should be consecutive without any gap. However, it is unessential for an LCS to be consecutive (contiguous).

Unlike $R O U G E_{N}$ which is computed using the recall value, $R O U G E_{L}$ is a combination of precision $P_{L C S}$ and recall $R_{L C S}$ values, which are defined in the following formulas:

$$
\begin{aligned}
P_{L C S} & =\max _{j \leq m} \frac{L C S\left(c_{i}, r e f_{i j}\right)}{\left|c_{i}\right|} \\
R_{L C S} & =\max _{j \leq m} \frac{L C S\left(c_{i}, r e f_{i j}\right)}{\left|r e f_{i j}\right|},
\end{aligned}
$$

where $m$ is the number of given references for a candidate $c_{i}$ and $\left|r e f_{i j}\right|$ is the number of words in the reference $r e f_{i j}$.

Now, we can compute the $R O U G E_{L}$ score for the candidate $c_{i}$ as the weighted 
harmonic average of both precision and recall as follows:

$$
R O U G E_{L}=\frac{\left(1+\beta^{2}\right) R_{L C S} P_{L C S}}{R_{L C S}+\beta^{2} P_{L C S}}
$$

where $\beta$ is a hyperparameter used to weigh the contribution of precision and recall values based on the application. For example, $\beta=1.2$ implies a higher weight is assigned to the recall value (emphasize the role of false negatives).

\subsection{CIDEr}

CIDEr stands for Consensus-based Image Description Evaluation [63, 51]. It is a new evaluation metric that was mainly developed for image captioning. The main purpose of this metric is to assess the quality of a generated candidate caption by comparing it with the consensus reference captions (descriptions generated by humans). However, it was used recently to assess iVQA [1].

Before computing CIDEr, some preprocessing steps are required. We first determine the stems (roots) of all words in candidate and reference sentences. For each sentence, we retrieve a set of the possible 1-to-4 grams representing the corresponding sentence. Following this, we compute the n-grams matches between the candidate sentence and reference sentences. However, different from other metrics like BLEU with which all matching n-grams are considered equally important irrespective of their relevance, CIDEr solves this issue by downweighting the n-grams that are frequent over the majority of reference sentences. To achieve this, CIDEr uses the Term Frequency-Inverse Document Frequency (TF-IDF) score. TF-IDF is widely used in

information retrieval systems. It combines both term frequency (TF) and inverse 
document frequency (IDF) scores to determine the importance of a given word in a set of sentences. Specifically, the term frequency upweights terms appearing very often in reference questions while the inverse document frequency controls this by downweighting terms if they appear over all given questions. As a result, terms like "the", "a", and other stopwords that are not only present in some questions but in most of the questions, would have low TF-IDF scores. In contrast, informative terms that are usually restricted to a few question sentences would have a high TF-IDF score. We compute the TF-IDF score, $g_{k}\left(r e f_{i j}\right)$ for each n-gram $w_{k}$, by applying the following formula:

$$
g_{k}\left(r e f_{i j}\right)=\frac{h_{k}\left(r e f_{i j}\right)}{\sum_{w_{l \in \Omega} h_{l}\left(r e f_{i j}\right)}} \log \left(\frac{|I|}{\sum_{I_{P} \in I} \min \left(1, \sum_{q} h_{k}\left(r e f_{p q}\right)\right)}\right),
$$

where $h_{k}\left(r e f_{i j}\right)$ is the number of an n-gram $w_{k}$ appearing in the reference $r e f_{i j}, \Omega$ is the set of all n-grams appearing in the reference $r e f_{i j}$, and $I$ denotes the set of all images. The first term in Eq. (5.13) represents the term frequency (TF) which weighs the n-gram $w_{k}$ in a reference $r e f_{i j}$, the second term is the inverse document frequency (IDF) which determines the prevalence of the n-gram $w_{k}$ across all reference questions by taking the logarithm of the number of images $(I)$ divided by the number of images where the n-gram $w_{k}$ appears in their corresponding reference questions.

After computing the TF-IDF scores for both candidate and reference sentences, we can compute the CIDEr score as follows:

$$
\operatorname{CIDEr}_{n}\left(c_{i}, R E F_{i}\right)=\frac{1}{m} \sum_{j} \frac{g^{n}\left(c_{i}\right) \cdot g^{n}\left(r e f_{i j}\right)}{\left\|g^{n}\left(c_{i}\right)\right\|\left\|g^{n}\left(r e f_{i j}\right)\right\|}
$$


where $c_{i}$ and $R E F_{i}$ represent candidate sentence $i$ and its corresponding reference sentences $R E F_{i}=\left\{r e f_{i 1}, \ldots, r e f_{i m}\right\}$ respectively, $g^{n}\left(c_{i}\right)$ is a vector obtained by computing the TF-IDF score $g_{k}\left(c_{i}\right)$ for all n-grams with size $n$ appearing in the candidate sentence $c_{i}$, and $\left\|g^{n}\left(c_{i}\right)\right\|$ is the magnitude of $g^{n}\left(c_{i}\right)$. The same definitions apply to $g^{n}\left(r e f_{i j}\right)$.

Similar to some other metrics, we use different lengths of n-grams (e.g. $\mathrm{n}=1,2,3,4)$ to enhance grammatical correctness and fluency. To account for that, the combined CIDEr score is defined as follows:

$$
\operatorname{CIDEr}\left(c_{i}, R E F_{i}\right)=\sum_{n=1}^{N} w_{n} C I D E r_{n}\left(c_{i}, R E F_{i}\right),
$$

where $N=4$ and $w_{n}=\frac{1}{N}$ is a constant weight.

CIDEr-D: is a variant of CIDEr. It was proposed by Vedantamet al. [63] to improve the correlation between CIDEr and human judgment. To achieve that, the authors eliminated the stemming step to give the metric more discriminative capabilities (e.g. to differentiate between plural and singular words). Furthermore, the modified CIDEr metric includes a length penalty to handle big length differences between candidate and reference sentences. Based on that, the new $C I D E r-D_{n}$ score is given as follows:

$C I D E r-D_{n}\left(c_{i}, R E F_{i}\right)=\frac{10}{m} \sum_{j} e^{\frac{-\left(l\left(c_{i}\right)-l\left(\text { ref }_{i j}\right)\right)^{2}}{2 \sigma^{2}}} \times \frac{\min \left(g^{n}\left(c_{i}\right), g^{n}\left(r e f_{i j}\right)\right) \cdot g^{n}\left(r e f_{i j}\right)}{\left\|g^{n}\left(c_{i}\right)\right\|\left\|g^{n}\left(r e f_{i j}\right)\right\|}$,

where $m$ is the number of given references, $l\left(c_{i}\right)$ represents the candidate's length, $l\left(r e f_{i j}\right)$ is the corresponding reference's length, and $\sigma$ is set to 6 . Note that the number of terms of the candidate is clipped by that of corresponding reference to 
avoid inaccurate high scores resulting from long sentences.

For different sizes of n-grams, the CIDEr $-D_{n}$ score is computed as follow: ${ }^{1}$

$$
C I D E r-D\left(c_{i}, R E F_{i}\right)=\sum_{n=1}^{N} w_{n} C I D E r-D_{n}\left(c_{i}, R E F_{i}\right),
$$

where $\mathrm{N}=4$ and $w_{n}=\frac{1}{N}$.

\footnotetext{
${ }^{1}$ We adopt CIDEr-D for our model evaluation
} 


\section{Chapter 6}

\section{Experiments}

In this section, we present our empirical study and report both quantitative and qualitative results.

\subsection{Experimental Setting}

\subsubsection{Dataset}

Visual Genome. The Visual genome dataset consists of 108K images. For each image, there is an average of 35 objects and 26 attributes. The objects are localized within bounding boxes. The dataset also provides a dense image annotation at the level of objects. Compared to the MS COCO which can only localize 80 unique objects, this dataset can localize over $100 \mathrm{~K}$ objects and attributes. Therefore, it is used by Anderson et al. [4] to train the Faster R-CNN image encoder adopted in our model. During training, only 1,600 objects and 400 attributes are considered to generate the object-based semantic and visual representations.

VQA V1. Simialr to [1, we used the VQA V1 dataset [15] to conduct experi- 
ments and evaluate the proposed approach. This dataset was initially created for the task of visual question answering and repurposed for iVQA [1] later. The images of VQA are collected from the MS COCO dataset [51]. This dataset is divided into three parts: the training set, validation set and testing set. The training set consists of 82,783 images. The validation and testing datasets include 40,504, and 81,434 images respectively. The answers, however, are not available for the testing set. Therefore, we followed [1, 4, 64] to use the Karpathy data split in which the validation set is split into 5,000 images for testing and 5,000 images for validation. Following [1], we use three question-answer pairs for each image. This results in about 250k instances [(image, answer, question)-triplets] for training and 15k instances for testing.

\subsubsection{Implementation Details}

Before conducting experiments, some data pre-processing steps are performed. Specifically, we follow [65] to extract the most frequent 3,000 answers from the training set and then build the vocabulary from the questions corresponding to these answers. This leads to a vocabulary set of about 12,900 words. The answers and questions are tokenized based on white space into lists of words. We prepend 0 to each question $\left(q_{0}=0\right)$ to indicate its beginning while we use the question mark "?" to denote the end of a question. We standardize the lengths of questions and answers to be $T=14$ and $m=3$ respectively by padding zeros to shorter sequences and trimming longer sequences. Subsequently, the answers tokens and semantic information, including both attributes and objects, are encoded into 300-dimensional vectors by using GloVe [55]. With regards to the questions' tokens, they are first one-hot encoded and then applied into a word embedding network initialized with GloVe.

To extract the visual and semantic information, we use the publicly available bottom- 
up attention model, Faster R-CNN image encoder, in [4]. We utilize the model to extract the top $k=36$ salient regions of visual features $V \in R^{36 \times 2048}$ and their corresponding semantic information $S \in R^{36 \times 300}$.

We use one NVIDIA Tesla V100 GPU with 16 GB of memory for training. As the size of data is large, it can not fit into the GPU memory. Therefore, the data is randomly fed in batches of 1,000 instances. In our SMLA model, we use $H=1280$, $N_{h}=1280$ hidden units for each GRU and $N=512$ for each attention module. In contrast, in the DMLA model, we use $H=1600, N=512$ for the dynamic attention and $N_{s}=256$ for the sequence attention modules. Regarding the MFB operation, we apply the same settings used in [37]. Specifically, we use $N_{f}=5000$ for the expanding step and $N_{z}=1000$ for the squeeze step. During training, we initially set the learning rate to $9.9 e^{-4}$. At the end of the fifth epoch, we decreased it to $9.9 e^{-5}$. To ensure generalization and reduce overfitting, we stop the training at the 15 th and 17th epochs ${ }^{1}$ for the SMLA and DMLA models, respectively. We train the models by using the back-propagation algorithm with the Adam [17] optimizer.

\subsubsection{Model Evaluation}

We evaluate our models by using standard evaluation metrics. Specifically, BLEU, METEOR, ROUGE-L and CIDEr? These metrics are used extensively to evaluate image caption models [51] and they can also be leveraged for evaluating similar sequence prediction tasks like iVQA [1, 2]. With the exception of CIDEr, the values of BLEU-(1,2,3,4), METEOR, ROUGE-L range from 0 to 1 , where 1 indicates that the generated questions are identical to the reference questions while 0 denotes that the

\footnotetext{
${ }^{1}$ Here the epoch means that we randomly feed our models with 248 batches. Hence, the model may not see the whole training set in an individual epoch.

${ }^{2}$ https://github.com/tylin/coco-caption
} 
Table 6.1: Overall performance comparison between our iVQA models and other baseline and state-of-the-art models on the popular Karpathy test split. Our models achieve best results on most evaluation metrics. The numbers in bold represent the highest scores (best performance).

\begin{tabular}{|l|c|c|c|c|c|c|c|}
\hline Method & BLEU-1 & BLEU-2 & BLEU-3 & BLEU-4 & METEOR & ROUGE-L & CIDEr \\
\hline SAT [1] & 0.417 & 0.311 & 0.241 & 0.192 & 0.195 & 0.456 & 1.533 \\
iVQA+VAE [2] & 0.421 & 0.320 & 0.253 & 0.205 & 0.201 & 0.466 & 1.682 \\
iVQA [1] & 0.430 & 0.326 & 0.256 & 0.208 & 0.205 & 0.468 & $\mathbf{1 . 7 1 4}$ \\
\hline Ours (DMLA) & 0.451 & 0.338 & 0.261 & 0.206 & 0.209 & 0.471 & 1.677 \\
\hline Ours (full SMLA) & $\mathbf{0 . 4 5 3}$ & $\mathbf{0 . 3 4 0}$ & $\mathbf{0 . 2 6 4}$ & $\mathbf{0 . 2 0 9}$ & $\mathbf{0 . 2 0 9}$ & $\mathbf{0 . 4 7 1}$ & 1.690 \\
\hline
\end{tabular}

generated questions and references are completely different. Regarding the CIDEr score, it ranges from 0 to 10 , where 10 represents the best possible score.

\subsection{Quantitative Analysis}

To validate the effectiveness of our proposed approaches, we compare our full SMLA and DMLA models with one baseline and two iVQA competitors [1, 2]. The baseline is the powerful Show Attend and Tell (SAT) model [39], which was mainly developed for the task of image captioning and modified by [1] to take an answer cue. The model is compared to our DMLA model in the context of using dynamic attention to focus on different salient regions at each time step. The competitors are the original iVQA [1] and its modified version (iVQA+VAE [2]) which incorporated the variational autoencoder (VAE) to increase the question diversity. We report the overall results for the instances in the test set in Table 6.1. From the comparison scores, we can see that the proposed SMLA and DMLA models with the multi-level attention mechanism perform better than the baseline SAT by remarkable margins. This suggests that the iVQA task presents different challenges than the caption generation task, while a state-of-the-art image captioning model can fail to produce effective results on the iVQA task even after adaptation. Moreover, we can see that our models also 
outperforms the state-of-the-art iVQA, iVQA and iVQA+VAE, in terms of most of the linguistic evaluation metrics, including BLEU-1, BLEU-2, BLEU-3, METEOR, ROUGE-L, and even BLEU-4 with our SMLA model. The results are also achieved under an inference setting that is favourable to the competitors - the two competitors used a beam search of size 3, while we only used a beam search of size 1 . These impressive results, that demonstrate the efficacy of our proposed static and dynamic multi-level attention models, can be explained by the following major factors. (1) Our usage of the powerful object-based visual representation is more suitable for iVQA as it facilitates the correlation between the given answer and corresponding visual objects. (2) Different from the competitors, we adopt the powerful MFB fusion method to directly capture the complex image-answer relationship and adopt MFB attention to attend to the generated image-answer fused features instead of only attending to the image. Furthermore, our full SMLA model leverages two additional answer-based visual and semantic attention modules. The visual attention with MFB attention could assist the model to consider different important regions needed to generate accurate questions that involve asking about one or several objects without even the need to use dynamic attention as in [1]. On the other hand, our DMLA model, which leverages attention modules for question encoding and decoding, also demonstrates

great results and proves that the proposed GRU-free question generation module used in our DMLA is promising for the iVQA task.

\subsection{Ablation Study}

In this section, we compare the performance of different variants of our SMLA and DMLA models. 


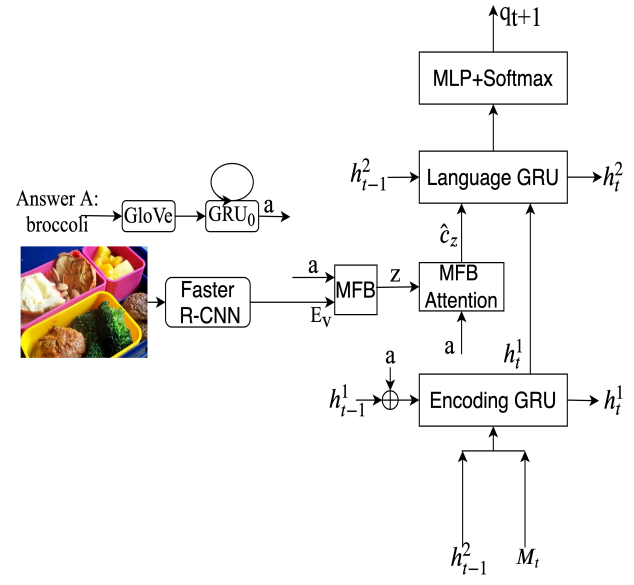

(a) Our baseline model

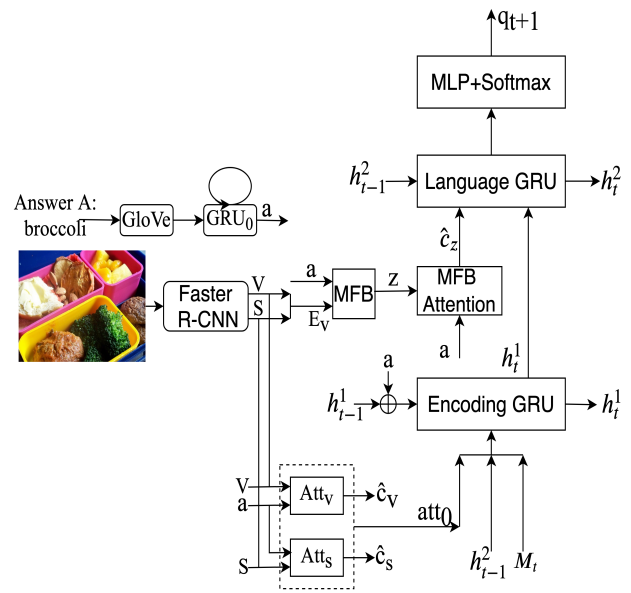

(b) Our full model

Figure 6.1: The architectures of our baseline SMLA model and full SMLA model

Performance Comparison of SMLA Model Variants. The proposed SMLA model uses two auxiliary visual and semantic attention modules. It leverages the additional knowledge represented by $S$ to produce enhanced visual features $E_{v}$ and in the semantic attention module $a t t_{s}$. To investigate the contribution of the main components in the major performance gain, we create the following variants of the proposed model.

1. Baseline Model. The baseline model is shown in Figure 6.1a. It first encodes a given answer using a GRU and encodes an image using Faster R-CNN. The model adopts MFB to fuse the answer cue with the object-based visual features. Subsequently, it uses the MFB attention to generate a weighted fused feature that is then injected to the model at each time step. Finally, a two-layer GRU model is used to recurrently generate questions.

2. Baseline Model with Visual Attention $\left(A t t_{v}\right)$. This variant represents our full model but without the use of the additional semantic information. With this model, we demonstrate the effect of using the auxiliary visual attention 
Table 6.2: Performance comparison of our SMLA model variants.

\begin{tabular}{|l|c|c|c|c|c|c|c|}
\hline Method & BLEU-1 & BLEU-2 & BLEU-3 & BLEU-4 & METEOR & ROUGE-L & CIDEr \\
\hline Baseline model & 0.449 & 0.336 & 0.259 & 0.204 & 0.206 & 0.465 & 1.625 \\
Baseline model+Att & 0.451 & 0.338 & 0.261 & 0.206 & 0.208 & 0.469 & 1.663 \\
Full SMLA model & $\mathbf{0 . 4 5 3}$ & $\mathbf{0 . 3 4 0}$ & $\mathbf{0 . 2 6 4}$ & $\mathbf{0 . 2 0 9}$ & $\mathbf{0 . 2 0 9}$ & $\mathbf{0 . 4 7 1}$ & $\mathbf{1 . 6 9 0}$ \\
\hline Full SMLA model+modified MFB attention & 0.451 & 0.338 & 0.261 & 0.206 & 0.208 & 0.472 & 1.668 \\
\hline
\end{tabular}

$A t t_{v}$ in improving the performance scores.

3. Full SMLA Model. By comparing our full model with the previous baseline model $+A t t_{v}$, we can see how much the additional semantic information $S$ contributes to the performance gain of our SMLA model.

4. Full SMLA Model with Modified MFB Attention. This model is similar to our full SMLA model. However, in this model, we modify the MFB attention to attend to the visual features rather than the image-answer fused features as in our full SMLA model. The main reason for this variant is to examine the effect of attending to the image-answer fused features $z$ instead of the visual features $V$ as adopted in the traditional visual attention. We compared the aforementioned variants and reported the results in Table 6.2.

From these results, we can see that the performance of the baseline model is comparable to the state-of-the-art iVQA model [1]. It actually outperforms the iVQA model in terms of BLEU-1, BLEU-2, BLEU-3 and METEOR. This validates our assumption that the visual features extracted at the object-level salient regions are more suitable for the task of iVQA than the visual features extracted with conventional deep networks (e.g. ResNet or VGG). Regarding the contribution of the visual attention $A t t_{v}$, we can see its effect in the second variant, the baseline model with visual attention $A t t_{v}$, where it is notable that $A t t_{v}$ assists the model to perform better especially in terms of ROUGE-L and CIDEr with an improvement of around $0.9 \%$ and $2.3 \%$ respectively. The additional semantic information, on the other hand, is also beneficial 
for our SMLA model. Its positive impact is clearly seen in the BLEU-4 score, where it helps our model to beat the state-of-the-art iVQA model [1] in terms of this important metric. Consequently, both the visual attention $A t t_{v}$ and semantic information $S$ are beneficial to boost the performance of our SMLA model. Specifically, the visual and semantic attention can be seen as auxiliary attention that enforces the model to recurrently consider the salient visual and semantic information corresponding with a given answer and at different levels of knowledge.

In the last variant, the full SMLA model with modified MFB attention, we can see that although the use of MFB attention to attend to visual features $V$ is beneficial, the significant impact of the proposed attention modules is more remarkable by attending to the rich image-answer fused features and not the visual features. This could be due to two major factors. Firstly, the model already attends to the visual features $V$ using the visual attention $A t t_{v}$. Consequently, the use of MFB attention weights to attend to the same visual features may be redundant. Secondly, the imageanswer fused features $z$ represent combinations of the semantic, visual, and answer cues. Hence, when we use the MFB attention weights to attend to $z$, we provide our model with a different level of knowledge represented by the necessary semantic, visual, and answer information.

Performance Comparison of DMLA Model Variants. Different from the SMLA model, the DMLA model does not use visual attention $A t t_{v}$ and additional semantic information $S$. It uses dynamic attention and sequence attention modules. The main purpose of this model is to examine the effect of using the attention mechanism for sequence learning as a possible powerful alternative for GRUs. To this end, we create a variant of the DMLA model, namely the hybrid DMLA model. This variant combines both attention modules and a GRU for question encoding and de- 
Table 6.3: Performance comparison of our DMLA model variants.

\begin{tabular}{|l|c|c|c|c|c|c|c|}
\hline Method & BLEU-1 & BLEU-2 & BLEU-3 & BLEU-4 & METEOR & ROUGE-L & CIDEr \\
\hline DMLA & 0.451 & 0.338 & 0.261 & 0.206 & 0.209 & 0.471 & 1.677 \\
Hybrid DMLA model & 0.452 & 0.338 & 0.262 & 0.207 & 0.208 & 0.468 & 1.660 \\
\hline
\end{tabular}

coding, respectively. In Table6.3, we report the results of both DMLA and its variant. From these results, we can see that both DMLA models achieve comparable scores. This validates our assumption that attention modules can replace GRUs in the task of iVQA without any negative effects on the performance. Importantly, the performance of our DMLA variants is, to some extent, close to that of our best performing SMLA model. However, the number of trainable parameters used in the DMLA model is around 9\% less than that used in the SMLA model. This again verifies that the use of attention modules to replace GRUs is promising.

\subsection{Qualitative Analysis}

To illustrate the function of our models' attention mechanisms in generating corresponding questions for given image-answer pairs, we demonstrate a few examples of the question generation process of our proposed approaches with attention visualization ${ }^{3}$ In Table 6.4 we visualize the attention weights of both the visual attention $A t t_{v}$ and the MFB attention $A t t_{m f b}$ used in our SMLA model. Besides that, we report the top-5 semantic objects selected by the semantic attention $A t t_{s}$. In Figure 6.2, we visualize the attention weights generated by the dynamic attention modules used in our full DMLA model.

Qualitative Results Discussion. According to the results in Table 6.4 and Figure

\footnotetext{
${ }^{3}$ Our attention visualization code is modified from https://github.com/peteanderson80/ bottom-up-attention
} 
Table 6.4: Qualitative results of our full SMLA model including a visualization of the weights generated from visual attention $A t t_{v}$ and MFB attention $A t t_{m f b}$ with the top-5 semantic objects selected by our semantic attention module $A t t_{s}$. Regarding $A t t_{v}$ and $A t t_{m f b}$, the red boxes mark the salient regions with the largest and second largest attention weights. Regions are gradually converted to white based on their attention weights where completely white regions mean very low attention weights. Regarding $A t t_{s}$, the text in bold denotes the semantic information that we believe it is important for the generation process.

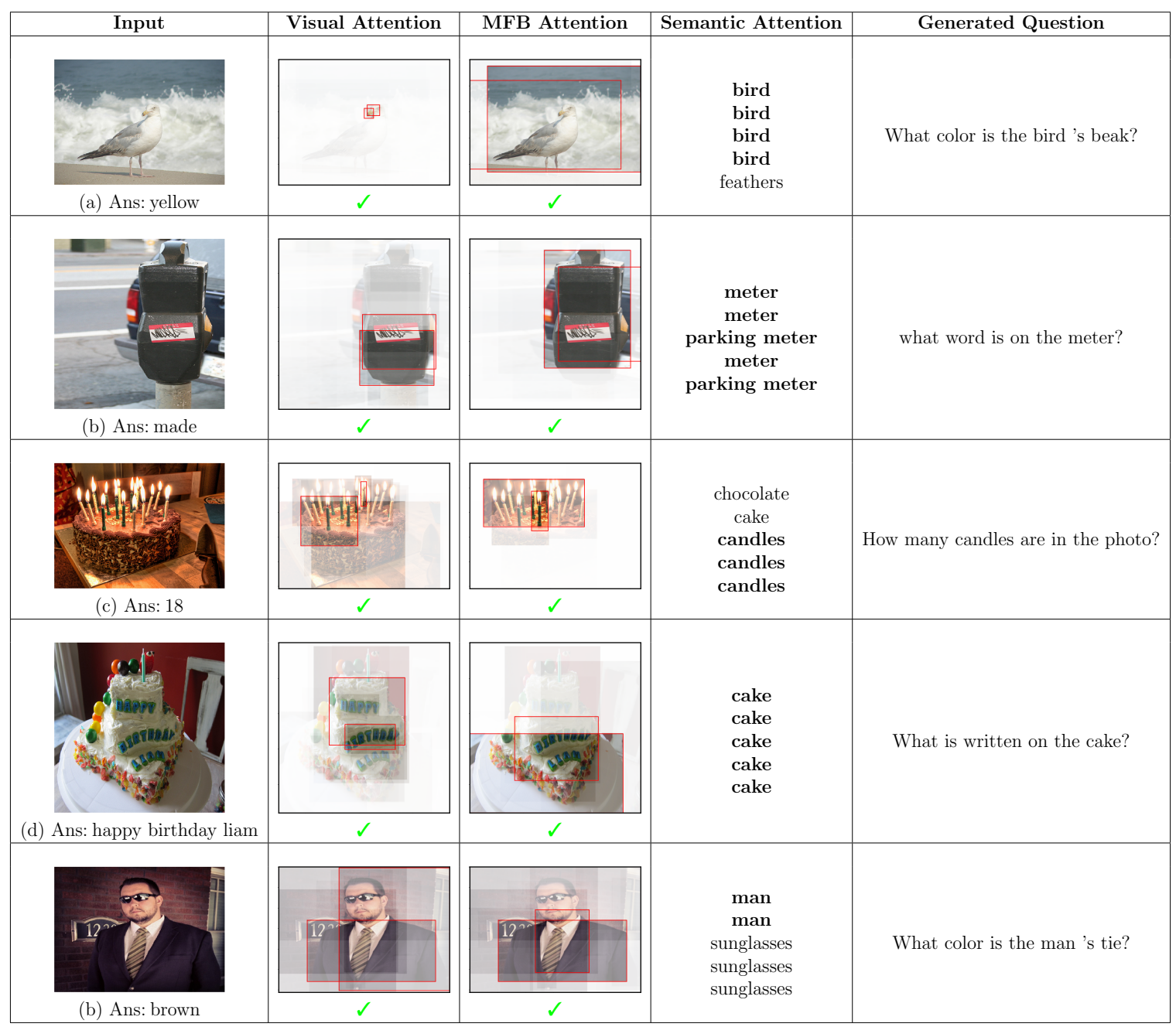




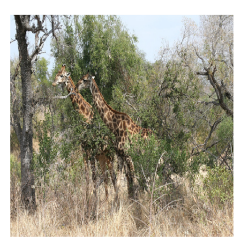

(a) Ans: 2

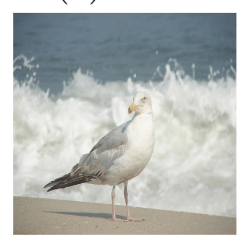

(b) Ans: yellow

(d)Ans:bow tie

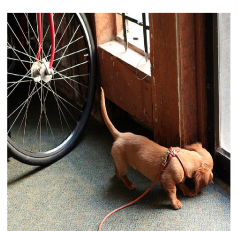

(d)Ans:harness
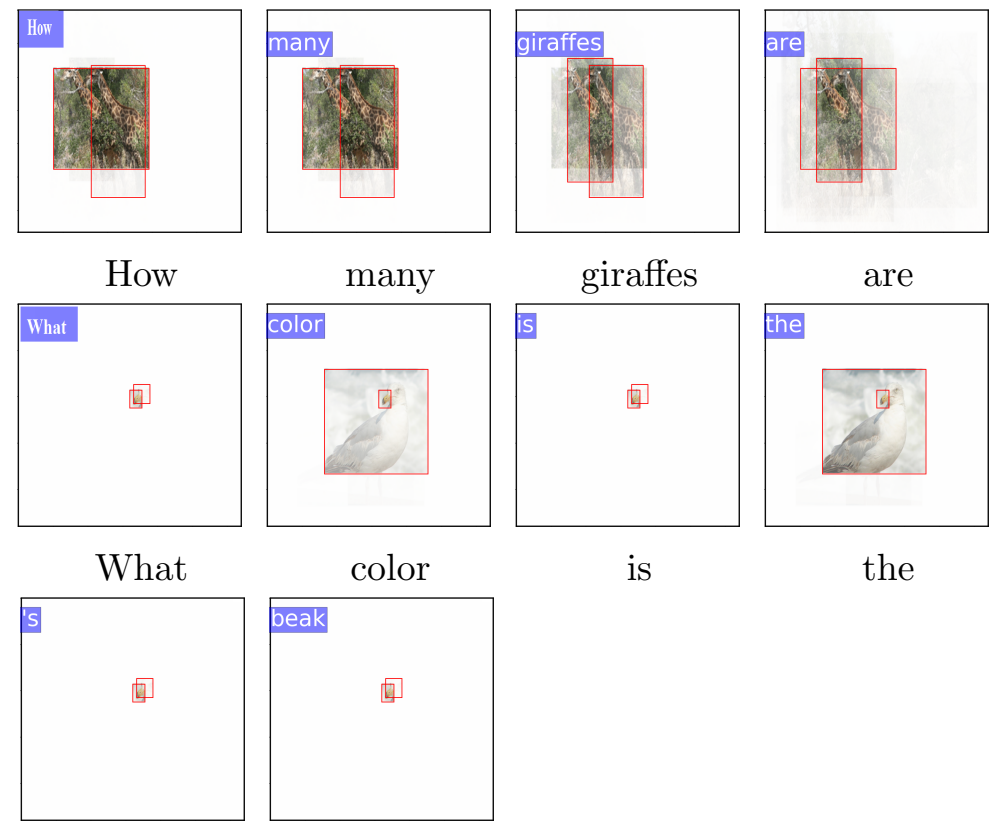

is

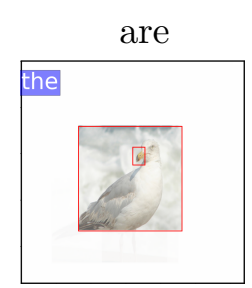

the
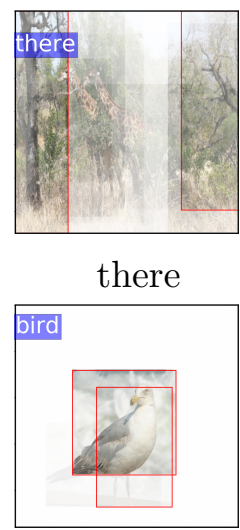

bird
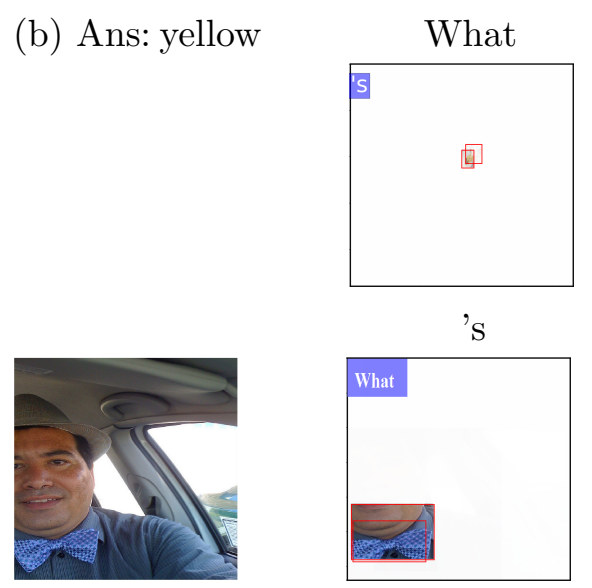

What

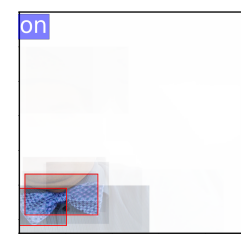

on

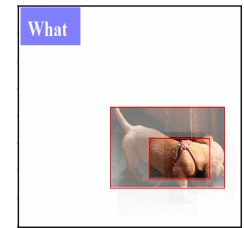

What beak

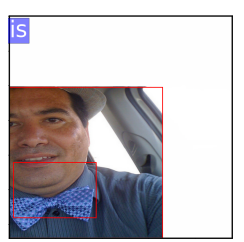

is

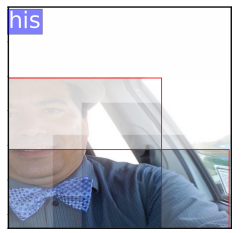

his

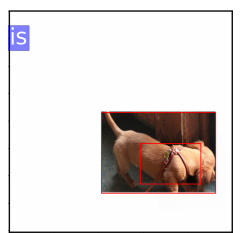

is

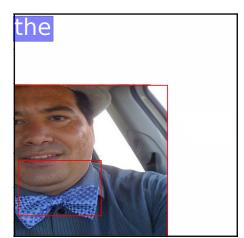

the

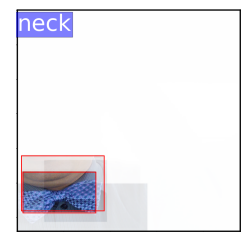

neck

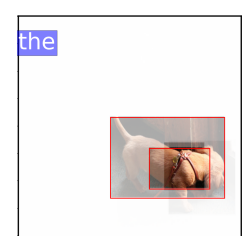

the

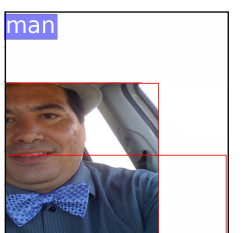

man

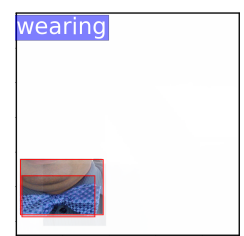

wearing

Figure 6.2: Examples of generated questions by our full DMLA model with corresponding dynamic attention visualization (The red bounding boxes localize Regions with the best and second best attention weights. Regions are gradually converted to white based on their attention weights where completely white regions mean very low attention weights). 
6.2, we can see that our SMLA and DMLA models can attend to salient regions in the images based on the given answers. Overall, the DMLA model seems to be better at attending to the important parts of images using dynamic attention. Nevertheless, this could be compensated in the SMLA model by integrating three static attention modules that attempt to capture various salient regions of images at different levels of knowledge.

Regarding our SMLA model, the complementary relationship of the attention modules used in the proposed static attention mechanism can be clearly seen in Table 6.4. In the first example, we notice that the model needs to attend to two salient objects, the bird and its beak, to generate the question "What color is the bird's beak". While the dynamic attention is not used, the SMLA model handles that by attending to the bird in general using the MFB and semantic attention, and to the bird's beak using the visual attention. Similarly, in the second example, we can see that the MFB and semantic attention mainly focus on the parking meter while the visual attention gives more priority to the written word on the meter. In the third example, although the semantic attention chooses the required object "candles" to be among the top-5 objects, it unnecessarily gives the highest priority for the "chocolate" and "cake" objects. However, we can see that the MFB and visual attention both focus on the candles from different views. Consequently, the model generates the correct question "How many candles are in the photo?" for the given answer "18". Similarly, we can see the mutual relationship between the applied attention modules in the last two examples. Based on the previous observations, we can conclude that it is not always necessary to use dynamic attention and at each time step to generate correct questions. Nevertheless, It could be sufficient to just consider salient objects associated with a given answer at the first time step. However, due to the complexity of the task of iVQA in locating the exact salient objects associated with given an- 
swers, it is beneficial to consider the image-answer relationship from different levels of knowledge by integrating different attention modules as proposed in our SMLA model.

Unlike the SMLA model, the DMLA model adopts the dynamic attention while generating the question's words, as shown in Figure 6.2. Specifically, the model is capable of dynamically changing its attention based on the answer and the generated question so far. To illustrate, in the first example where the given answer is "2", the model can understand that the question should be about something involving two objects. Therefore, while generating the word giraffes it only focuses on two giraffes in the image and completely ignores other parts. The role of dynamic attention can be clearly noted in the second example, where the model only attends to the bird object while generating the word "bird" and the bird's beak while generating the word "beak". In this example, the dynamic attention outperforms the MFB attention used in our SMLA model. This is can be especially seen when the dynamic attention generates the word "bird". Although the MFB attention in the SMLA model is, to some extent, able to attend to the bird, our DMLA model was more accurate in attending to the bird object without including any additional unnecessary information. The ability of our dynamic attention mechanism to attend to the fine-grained details of an image given both an answer and a partial generated question is further verified in the third example. We can see that the model generates a very suitable question for the given answer "bow tie" while dynamically attending to different salient objects (e.g. , man, bow tie, and neck). In the last example, we examine the robustness of our model by applying a more challenging answer (harness). We notice that the model correctly attends to the dog object when it generates the word "dog". However, although the model inaccurately gives high importance to the bike's wheel, it can attend to the harness object while generating the word "wearing". These examples show that 
our approach has the capacity to correlate a given answer with specific corresponding regions in a given image. Meanwhile, it also has the flexibility to dynamically change its attention according to the generated word. These results again validated the effectiveness of the proposed model.

\subsection{Failure Analysis}

Although the SMLA and DMLA models are capable of generating corresponding questions for a given image and answer pair, they experience some failure cases that need to be handled in order to have a complete solution for the task of iVQA.

Firstly, although the attention mechanisms used in our SMLA and DMLA models demonstrate great effectiveness regarding the capability of attending to fine-grained details of an image based on a given answer, they still have some limitations in some aspects. Specifically, the static multi-level attention mechanism used in our SMLA model sometimes fails to attend to the required answer-based objects, as shown in the first example of Table 6.4. In this example, the MFB attention attends to the bird object, which is relevant. However, it inaccurately focuses on unnecessary information with the bird. This failure could be explained in two aspects. (1) Faster R-CNN generated visual features representing the bird object, but some of these features were not exactly aligned with the bird. Consequently, the MFB attention attends to one of these misaligned features. (2) The MFB attention is responsible to weigh the fused image-answer features $z$. Each one of these enriched features represents a combination of the input visual and textual information. We think that it is complex to select the relevant visual and textual information needed for the generation process using one attention module. Therefore, when the MFB attention fails to decide the relevant visual and textual information, it sometimes tends to be more generalized 
and attends to some irrelevant features. The semantic attention, on the other hand, also fails in the third example of Table 6.4, where it considers the chocolate and cake concepts to be more important than the candle concept. In such cases, we think that the model is more affected by the general salient semantic concepts compared to those answer-aware concepts. With regards to our DMLA model, the applied dynamic attention experiences some limitations that can be seen in the last example of Figure 6.2. where the model successfully attends to some corresponding objects (e.g. , dog and harness), but it inaccurately attends to the wheel object which is irrelevant for the generated question.

Secondly, generating accurate questions for Yes/No answers or answers involving numbers is another challenge for our models. Unlike VQA task in which the intelligent system utilizes more information involved in a given question to predict correct answers, the information provided by the answer is limited and sometimes involves enormous possibilities regarding the generated questions, which may negatively affect the ability of the model to generate correct questions. Some examples are demonstrated in Table 6.5. We can see that in the first two examples our models generate corresponding and correct questions for given "yes" and "no" answers. However, none of them is identical to the ground-truth questions. This degrades the performance of our models, which are mainly evaluated based on the co-existence of n-grams in the generated and ground-truth questions. Regarding the answers involving numbers, we can see that our models generate inaccurate questions, as shown in the last two examples of Table 6.5. In example (c), the models understand that there are 6 donuts and generate reasonable questions, but this is not as specific as the ground-truth question which accurately asks about the donuts with sprinkles. Similarly, in the example (d), the models ask about the number of elephants in general without being precise to those "adult elephants" which is more related to the given answer "4". In fact, 
we think that this problem is very challenging as the models should first count the occurrence of each object in a given image instead of merely focusing on the number of some objects as in VQA, where the leading objects are usually involved in given questions.

Table 6.5: Examples of generated questions by our SMLA and DMLA models with their associated ground-truth questions for yes/no and number answers.

\begin{tabular}{|c|c|c|}
\hline Input & Ground Truth Question & Generated Question \\
\hline & & \\
\hline & Is this boy having fun? & SMLA:Is the boy wearing a helmet? \\
DMLA:Is the boy wearing a helmet?
\end{tabular}




\section{Chapter 7}

\section{Conclusion and Future Work}

In this thesis, we proposed the novel SMLA and DMLA models to tackle the new task of iVQA, which has a broader capacity for interacted visual and textual understanding than VQA. In our SMLA model, we develop a static multi-level attention mechanism which captures the different aspects of the complex image and answer relationship. Unlike the existing iVQA approaches, we enhance the visual representation extracted at the level of objects with their corresponding semantic information to reduce the semantic gap between visual and textual modalities. In addition, we deploy three attention modules: the visual and semantic attentions that help the model to focus on the salient visual and semantic objects corresponding to a given answer, and the MFB attention to capture the important information in the image-answer fused features before starting the question generation process.

On the other hand, in our DMLA model, we handle the task of iVQA from a different perspective by adopting a dynamic multi-level attention mechanism to attend to the important parts of the image-answer fused features in each recurrent question generation context. Different from SMLA and the other iVQA models, we develop a method for question encoding and decoding that is based on attention, to replace the 
conventional sequence learning methods(e.g. , RNNs and its variants). We conducted experiments on the VQA V1 dataset to compare the proposed models with existing iVQA methods. The results show the effectiveness of the proposed approaches by demonstrating state-of-the-art performance. Based on these impressive results, we have made the following principal observations:

1. Leveraging of object-based regional feature extraction model like Faster R-CNN is more suitable for the task of iVQA.

2. Fusing of image and answer representation using a bilinear pooling operation (MFB) and then attending to the extracted image-answer fused features rather than the image representation, is useful for iVQA.

3. The use of the visual and semantic attentions, with the MFB attention, is beneficial to signify visual and concepts features corresponding to a given answer.

4. It is not always essential to dynamically attend to an image to generate corresponding questions. In iVQA, unlike image captioning, we have found that integrating several static attention modules to capture the different aspects of image and answer relationship, as applied in our SMLA model, could be sufficient to generate corresponding questions. Consequently, this would save some parameters needed for the dynamic attention at each time step.

5. The attention mechanism that was mainly used to attend images, could also be used as a powerful alternative for the conventional RNNs in question encoding and decoding, as proposed in our DMLA model.

Future Work. As discussed in the section of failure analysis, many challenges need to be addressed to provide better models for the task of iVQA. 
With regards to the applied attention mechanisms, we plan to investigate other techniques to enhance their functionalities. For example, in the SMLA model, we think it may be useful to first train each attention module separately. Subsequently, we can use these pre-trained modules and fine-tune them with our full SMLA model. This transfer learning-based technique is used in our Faster R-CNN model which adopts ResNet101 pretrained on the ImageNet dataset. Moreover, we reckon the performance of the attention mechanisms could be also improved by applying other state-of-the-art attention mechanisms as in [43, 37]. The stacked attention network proposed in [43] is especially beneficial to enhance the selectivity of the attention mechanisms used in the SMLA model by attending to the image multiple times. In addition, we plan to investigate the use of the state-of-the-art adaptive attention [41] with our DMLA model. We think that this attention is suitable for iVQA, as some words in the generated question (e.g. of, a, an) are already known for the decoder and no visual attention is needed in these cases. Another interesting direction for future work is to investigate the use of a counting module [66] to promote the reasoning capabilities of the proposed models to generate accurate questions for answers involving numbers. 


\section{List of References}

[1] Feng Liu, Tao Xiang, Timothy M. Hospedales, Wankou Yang, and Changyin Sun. "IVQA: inverse visual question answering". In IEEE Conference on Computer Vision and Pattern Recognition (CVPR), pages 8611-8619, 2018.

[2] Feng Liu, Tao Xiang, Timothy M. Hospedales, Wankou Yang, and Changyin Sun. "Inverse visual question answering: A new benchmark and vqa diagnosis tool". IEEE transactions on pattern analysis and machine intelligence (TPAMI), pages $1-1,2018$.

[3] Hedi Ben-younes, Rémi Cadène, Matthieu Cord, and Nicolas Thome. "MUTAN: Multimodal tucker fusion for visual question answering". In IEEE International Conference on Computer Vision (ICCV), pages 2631-2639, 2017.

[4] Peter Anderson, Xiaodong He, Chris Buehler, Damien Teney, Mark Johnson, Stephen Gould, and Lei Zhang. "Bottom-up and top-down attention for image captioning and visual question answering". In IEEE Conference on Computer Vision and Pattern Recognition (CVPR), pages 6077-6086, 2018.

[5] Yuetan Lin, Zhangyang Pang, Donghui Wang, and Yueting Zhuang. "Feature enhancement in attention for visual question answering". In Proceedings of the Twenty-Seventh International Joint Conference on Artificial Intelligence (IJCAI), pages 4216-4222, 2018.

[6] Jin-Hwa Kim, Kyoung Woon On, Woosang Lim, Jeonghee Kim, Jung-Woo Ha, and Byoung-Tak Zhang. "Hadamard product for low-rank bilinear pooling". In 5th International Conference on Learning Representations (ICLR), 2017.

[7] Nasrin Mostafazadeh, Ishan Misra, Jacob Devlin, C. Lawrence Zitnick, Margaret Mitchell, Xiaodong He, and Lucy Vanderwende. "Generating natural questions about an image". ArXiv, abs/1603.06059, 2016.

[8] Shijie Zhang, Lizhen Qu, Shaodi You, Zhenglu Yang, and Jiawan Zhang. "Automatic generation of grounded visual questions". In Proceedings of the TwentySixth International Joint Conference on Artificial Intelligence (IJCAI), pages 4235-4243, 2017. 
[9] U. Jain, Z. Zhang, and A. Schwing. "Creativity: Generating diverse questions using variational autoencoders". In IEEE Conference on Computer Vision and Pattern Recognition (CVPR), pages 5415-5424, 2017.

[10] Yash Goyal, Tejas Khot, Douglas Summers-Stay, Dhruv Batra, and Devi Parikh. "Making the V in VQA matter: Elevating the role of image understanding in visual question answering". In IEEE Conference on Computer Vision and Pattern Recognition (CVPR), pages 6325-6334, 2017.

[11] Yikang Li, Nan Duan, Bolei Zhou, Xiao Chu, Wanli Ouyang, Xiaogang Wang, and Ming Zhou. "Visual question generation as dual task of visual question answering". In IEEE Conference on Computer Vision and Pattern Recognition (CVPR), pages 6116-6124, 2018.

[12] Kaiming He, Xiangyu Zhang, Shaoqing Ren, and Jian Sun. "Deep residual learning for image recognition". In IEEE Conference on Computer Vision and Pattern Recognition (CVPR), pages 770-778, 2015.

[13] K. Simonyan and A. Zisserman. "Very deep convolutional networks for largescale image recognition". In International Conference on Learning Representations (ICLR), 2015.

[14] Shaoqing Ren, Kaiming He, Ross B. Girshick, and Jian Sun. "Faster R-CNN: Towards real-time object detection with region proposal networks". IEEE Transactions on Pattern Analysis and Machine Intelligence (TPAMI), 39(6):1137-1149, 2015.

[15] Stanislaw Antol, Aishwarya Agrawal, Jiasen Lu, Margaret Mitchell, Dhruv Batra, C. Lawrence Zitnick, and Devi Parikh. "VQA: Visual Question Answering". In International Conference on Computer Vision (ICCV), pages 2425-2433, 2015 .

[16] Roger Parloff. "Why deep learning is suddenly changing your life". http: //fortune.com/ai-artificial-intelligence-deep-machine-learning/, 2016.

[17] Diederik P. Kingma and Jimmy Ba. "Adam: A method for stochastic optimization". In 3rd International Conference on Learning Representations (ICLR), 2015 .

[18] Luke Dormehl. "What is an artificial neural network? here's everything you need to know". https://www.digitaltrends.com/cool-tech/what-is-anartificial-neural-network/, 2019.

[19] Stanford University. "Neural networks". http://ufldl.stanford.edu/wiki/ index.php/Neural_Networks, 2013. 
[20] Kandeng. "Backpropagation algorithm". http://ufldl.stanford.edu/wiki/ index .php?title=Backpropagation_Algorithm\&oldid=2272, 2013.

[21] Ross B. Girshick. "Fast R-CNN". In IEEE International Conference on Computer Vision (ICCV), pages 1440-1448, 2015.

[22] Ross B. Girshick, Jeff Donahue, Trevor Darrell, and Jitendra Malik. "Rich feature hierarchies for accurate object detection and semantic segmentation". In IEEE Conference on Computer Vision and Pattern Recognition (CVPR), pages 580$587,2014$.

[23] Saha Sumit. "A comprehensive guide to convolutional neural networks-the ELI5 way". https://towardsdatascience.com/a-comprehensive-guide-toconvolutional-neural-networks-the-eli5-way-3bd2b1164a53, 2018.

[24] Andrej Karpathy. "CS231n convolutional neural networks for visual recognition". http://cs231n.github.io/convolutional-networks/, 2018.

[25] Max Ferguson, Ronay Ak, Y. Tina Lee, and Kincho H. Law. "Automatic localization of casting defects with convolutional neural networks". In IEEE International Conference on Big Data (Big Data), pages 1726-1735, 2017.

[26] Niklas Donges. "Recurrent Neural Networks and LSTM". https: //towardsdatascience. com/recurrent-neural-networks-and-lstm4b601dd822a5, 2018.

[27] Y. Bengio, P. Simard, and P. Frasconi. "Learning long-term dependencies with gradient descent is difficult". IEEE Transactions on Neural Networks, 5(2):157$166,1994$.

[28] Sepp Hochreiter and Jürgen Schmidhuber. "Long Short-Term Memory". Neural computation, 9(8):1735-1780, 1997.

[29] Kyunghyun Cho, Bart Van Merrienboer, Caglar Gulcehre, Dzmitry Bahdanau, Fethi Bougares, Holger Schwenk, and Yoshua Bengio. "Learning phrase representations using RNN encoderdecoder for statistical machine translation". In Proceedings of the 2014 Conference on Empirical Methods in Natural Language Processing (EMNLP), pages 1724-1734, 2014.

[30] Junyoung Chung, Çaglar Gülçehre, KyungHyun Cho, and Yoshua Bengio. "Empirical evaluation of gated recurrent neural networks on sequence modeling". CoRR, abs/1412.3555, 2014.

[31] Christopher Olah. "Understanding LSTM Networks". http://colah.github. io/posts/2015-08-Understanding-LSTMs/, 2015. 
[32] Jasper R. R. Uijlings, Koen E. A. van de Sande, Theo Gevers, and Arnold W. M. Smeulders. "Selective search for object recognition". International Journal of Computer Vision (IJCV), 104(2):154-171, 2013.

[33] Tomasz Grel. "region of interest pooling explained". https://deepsense.ai/ region-of-interest-pooling-explained/, 2019.

[34] Yu Liu, Yanming Guo, Erwin M. Bakker, and Michael S. Lew. "Learning a recurrent residual fusion network for multimodal matching". In IEEE International Conference on Computer Vision (ICCV), pages 4127-4136, 2017.

[35] Bolei Zhou, Yuandong Tian, Sainbayar Sukhbaatar, Arthur Szlam, and Rob Fergus. "Simple baseline for visual question answering". CoRR, abs/1512.02167, 2015 .

[36] Mingrui Lao, Yanming Guo, Hui Wang, and Xin Zhang. "Multimodal local perception bilinear pooling for visual question answering". IEEE Access, 6:5792357932, 102018.

[37] Zhou Yu, Jun Yu, Jianping Fan, and Dacheng Tao. "Multi-modal factorized bilinear pooling with co-attention learning for visual question answering". In IEEE International Conference on Computer Vision (ICCV), pages 1839-1848, 2017.

[38] Oriol Vinyals, Alexander Toshev, Samy Bengio, and Dumitru Erhan. "Show and tell: A neural image caption generator". In IEEE Conference on Computer Vision and Pattern Recognition (CVPR), pages 3156-3164, 2015.

[39] Kelvin Xu, Jimmy Ba, Ryan Kiros, Kyunghyun Cho, Aaron C. Courville, Ruslan Salakhutdinov, Richard S. Zemel, and Yoshua Bengio. "Show, attend and tell: Neural image caption generation with visual attention". In Proceedings of the 32nd International Conference on Machine Learning (ICML), pages 2048-2057, 2015.

[40] Quanzeng You, Hailin Jin, Zhaowen Wang, Chen Fang, and Jiebo Luo. "Image captioning with semantic attention". In IEEE Conference on Computer Vision and Pattern Recognition (CVPR), pages 4651-4659, 2016.

[41] Jiasen Lu, Caiming Xiong, Devi Parikh, and Richard Socher. "Knowing when to look: Adaptive attention via a visual sentinel for image captioning". In IEEE Conference on Computer Vision and Pattern Recognition (CVPR), pages 32423250, 2017.

[42] Kan Chen, Jiang Wang, Liang-Chieh Chen, Haoyuan Gao, Wei Xu, and Ramakant Nevatia. "ABC-CNN: An attention based convolutional neural network for visual question answering". CoRR, abs/1511.05960, 2015. 
[43] Zichao Yang, Xiaodong He, Jianfeng Gao, Li Deng, and Alexander J. Smola. "Stacked attention networks for image question answering". In IEEE Conference on Computer Vision and Pattern Recognition (CVPR), pages 21-29, 2016.

[44] Jiasen Lu, Jianwei Yang, Dhruv Batra, and Devi Parikh. "Hierarchical questionimage co-attention for visual question answering". In Conference on Neural Information Processing Systems (NIPS), pages 289-297, 2016.

[45] Dongfei Yu, Jianlong Fu, Tao Mei, and Yong Rui. "Multi-level attention networks for visual question answering". In IEEE Conference on Computer Vision and Pattern Recognition (CVPR), pages 4187-4195, 2017.

[46] Issey Masuda Mora and Santiago Pascual de la Puente. "Towards automatic generation of question answer pairs from images". In Conference on Computer Vision and Pattern Recognition Workshop (CVPRW), 2016.

[47] Nasrin Mostafazadeh, Chris Brockett, William B. Dolan, Michel Galley, Jianfeng Gao, Georgios P. Spithourakis, and Lucy Vanderwende. "Image-grounded conversations: Multimodal context for natural question and response generation". In Proceedings of the Eighth International Joint Conference on Natural Language Processing (IJCNLP), pages 462-472, 2017.

[48] Justin Johnson, Andrej Karpathy, and Li Fei-Fei. "Densecap: Fully convolutional localization networks for dense captioning". In IEEE Conference on Computer Vision and Pattern Recognition (CVPR), pages 4565-4574, 2016.

[49] Yining Li, Cheng Huang, Xiaoou Tang, and Chen Change Loy. "Learning to disambiguate by asking discriminative questions". In IEEE International Conference on Computer Vision (ICCV), pages 3439-3448, 2017.

[50] Diederik P. Kingma and Max Welling. "Auto-encoding variational bayes". In 2nd International Conference on Learning Representations (ICLR), 2014.

[51] Xinlei Chen, Hao Fang, Tsung-Yi Lin, Ramakrishna Vedantam, Saurabh Gupta, Piotr Dollár, and C. Lawrence Zitnick. "Microsoft COCO captions: Data collection and evaluation server". CoRR, abs/1504.00325, 2015.

[52] Feng Liu, Tao Xiang, Timothy M. Hospedales, Wankou Yang, and Changyin Sun. "Semantic regularisation for recurrent image annotation". In IEEE Conference on Computer Vision and Pattern Recognition (CVPR), pages 4160-4168, 2017.

[53] Ashish Vaswani, Noam Shazeer, Niki Parmar, Jakob Uszkoreit, Llion Jones, Aidan N. Gomez, Lukasz Kaiser, and Illia Polosukhin. "Attention is all you need". In Conference on Neural Information Processing Systems (NIPS), pages 5998-6008, 2017. 
[54] Jacob Devlin, Ming-Wei Chang, Kenton Lee, and Kristina Toutanova. "BERT: pre-training of deep bidirectional transformers for language understanding". In Conference of the North American Chapter of the Association for Computational Linguistics: Human Language Technologies (NAACL-HLT), pages 4171-4186, 2019.

[55] Jeffrey Pennington, Richard Socher, and Christopher D. Manning. "Glove: Global vectors for word representation". In Conference on Empirical Methods in Natural Language Processing (EMNLP), pages 1532-1543, 2014.

[56] Olga Russakovsky, Jia Deng, Hao Su, Jonathan Krause, Sanjeev Satheesh, Sean Ma, Zhiheng Huang, Andrej Karpathy, Aditya Khosla, Michael S. Bernstein, Alexander C. Berg, and Li Fei-Fei. "Imagenet large scale visual recognition challenge". International Journal of Computer Vision (IJCV), 115(3):211-252, 2015 .

[57] Ranjay Krishna, Yuke Zhu, Oliver Groth, Justin Johnson, Kenji Hata, Joshua Kravitz, Stephanie Chen, Yannis Kalantidis, Li-Jia Li, David A. Shamma, Michael S. Bernstein, and Li Fei-Fei. "Visual genome: Connecting language and vision using crowdsourced dense image annotations". International Journal of Computer Vision (IJCV), 123(1):32-73, 2017.

[58] Kishore Papineni, Salim Roukos, Todd Ward, and Wei-Jing Zhu. "Bleu: a method for automatic evaluation of machine translation". In Proceedings of the 40th Annual Meeting of the Association for Computational Linguistics (ACL), pages 311-318, 2002.

[59] Xiaoxiao Liu, Qingyang Xu, and Ning Wang. "A survey on deep neural networkbased image captioning". The Visual Computer, 35(3):445-470, 2019.

[60] Michael J. Denkowski and Alon Lavie. "Meteor universal: Language specific translation evaluation for any target language". In Proceedings of the Ninth Workshop on Statistical Machine Translation (WMT), 2014.

[61] George A. Miller. "WORDNET: A lexical database for english". Communications of the ACM, 38(11):39-41, 1995.

[62] Chin-Yew Lin. "ROUGE: A package for automatic evaluation of summaries". In Association for Computational Linguistics (ACL), pages 74-81, 2004.

[63] Ramakrishna Vedantam, C. Lawrence Zitnick, and Devi Parikh. "CIDEr: Consensus-based image description evaluation". In IEEE Conference on Computer Vision and Pattern Recognition (CVPR), pages 4566-4575, 2015. 
[64] Andrej Karpathy and Li Fei-Fei. "Deep visual-semantic alignments for generating image descriptions". In IEEE Conference on Computer Vision and Pattern Recognition (CVPR), pages 3128-3137, 2015.

[65] Jiasen Lu, Xiao Lin, Dhruv Batra, and Devi Parikh. "Deeper LSTM and normalized CNN visual question answering model". https://github.com/VT-vision1ab/VQA_LSTM_CNN, 2015.

[66] Yan Zhang, Jonathon S. Hare, and Adam Prügel-Bennett. "Learning to count objects in natural images for visual question answering". CoRR, abs/1802.05766, 2018. 